\title{
A simplified homogenized limit analysis model for randomly assembled blocks out-of-plane loaded
}

\author{
Gabriele Milani $^{(1)}$, Paulo B. Lourenço ${ }^{(2)}$ \\ (1) Corresponding Author. Dipartimento di Ingegneria Strutturale (DIS), Politecnico di Milano, \\ Piazza Leonardo da Vinci 32, 20133 Milano, Italy. E-mail: milani@ stru.polimi.it. \\ ${ }^{(2)}$ ISISE, Department of Civil Engineering, School of Engineering, University of Minho, Azurém, \\ 4800-058 Guimarães, Portugal. E-mail: pbl@ civil.uminho.pt.
}

\begin{abstract}
A kinematic rigid-plastic homogenization model for the limit analysis of masonry walls arranged in random texture and out-of-plane loaded is proposed. The model is the continuation of a previous work by the authors in which masonry in-plane behavior was investigated. In the model, blocks constituting a masonry wall are supposed infinitely resistant with a Gaussian distribution of height and length, whereas joints are reduced to interfaces with frictional behavior and limited tensile and compressive strength. Block by block, a representative element of volume (REV) is considered, constituted by a central block interconnected with its neighbors by means of rigid plastic interfaces. Two different classes of problems are investigated, the first consisting of full stochastic REV assemblages without horizontal and vertical alignment of joints, the second assuming the presence of a horizontal alignment along bed joints, i.e. allowing blocks height variability only row by row. A sub-class of elementary deformation modes is a-priori chosen in the REV, mimicking typical failures due to joint cracking and crushing. The model is characterized by a few material parameters and it is therefore particularly suited to perform large scale Monte Carlo simulations. Masonry strength domains are obtained equating the power dissipated in the heterogeneous model with the power dissipated by a fictitious homogeneous macroscopic plate. A stochastic estimation of out-ofplane masonry strength domains (both bending moments and torsion are considered) accounting for the geometrical statistical variability of blocks dimensions is obtained with the proposed model. The case of deterministic block height (quasi-periodic texture) can be obtained as a subclass of this latter case. As an important benchmark, the case in which joints obey a Mohr-Coulomb failure criterion is also tested and compared with results obtained assuming a more complex interfacial behavior for mortar. Masonry homogenized failure surfaces are finally implemented in an upper bound Finite Element (FE) limit analysis code. Firstly, to validate the model proposed, two small scale structural examples of practical interest are considered, relying in masonry panels in two-way out-of-plane bending. In both cases, failure load distributions and failure mechanisms provided by the homogenization model are compared with those obtained through a heterogeneous approach.

Finally, in order to show the capabilities of the approach proposed when dealing with large scale structures, the ultimate behavior prediction of a Romanesque masonry church façade located in Portugal and arranged in irregular texture is presented. Comparisons with Finite Element heterogeneous approaches and "at hand" calculations show that reliable predictions of the load bearing capacity of real large scale structures may be obtained with a very limited computational effort.
\end{abstract}

Keywords: Masonry, out-of-plane loads, simplified homogenization, random pattern, Monte Carlo simulations. 


\section{Introduction}

Masonry constituted by the assemblage of blocks and stones with variable dimensions, Figure 1, is very common in both existing and historical buildings in many countries. However, the complexity of the problem, and the number of variables required by accurate numerical heterogeneous FE analyses [1]-[4], usually preclude the study of these structures in the inelastic range through commercial software. In this context, it appears particularly interesting to present a numerical model based on Finite Elements (FEs) able to give reasonable estimations on the ultimate behavior of masonry walls constituted by an irregular assemblage of blocks and loaded out-of-plane.

At present, there is still a lack of knowledge concerning the behavior of masonry structures randomly assembled, despite the fact that several efforts have been recently done by many authors to tackle the problem using stochastic homogenization schemes.

As a rule, when dealing with a masonry structure constituted by a random assemblage of blocks, a key problem is in the definition and the use of a suitable material constitutive law at a macro-scale. Following a kinematic limit analysis approach proposed by the authors for in-plane actions [5], simplified homogenization concepts are used in this paper to obtain an estimation of random masonry out-plane failure surfaces. Homogenization [6]-[10] appears indeed a good compromise between micro and macro-modeling, as it allows to derive in a rational way stress-strain relationships for masonry, suitably taking into account texture and mechanical properties of the constituent materials only at a cell level. Moreover, it may lead to effective models, with reduced computational effort for real scale simulations (e.g. Pegon and Anthoine [6], Luciano and Sacco [7], Massart et al. [9], Mercatoris and Massart [10]). As a demonstration that homogenization seems to be the most suited tool for the analysis of stochastic assemblages of blocks, it is worth remembering that almost all models available in the literature for irregular masonry make use of homogenization concepts (see, for instance, [11]-[18]).

However, almost all these contributions deal with the analysis of irregular masonry within the linear elastic range [11]-[16]. Non-linear analyses on such kind of structures are very attractive, but would require to perform a full set of expensive FE Monte Carlo (MC) simulations, each one relying in a boundary value problem with non-linear constitutive laws for blocks and mortar. In this context, the prediction of the load bearing capacity of entire walls (e.g. mean resistance and collapse load distributions) remains a challenging task. Probably for this reason, a few papers are available in the technical literature focusing on the application of homogenization theory in the inelastic range combined with standard FE discretizations for random assemblages of blocks [17][18]. In any case, 
an implementation of a two-scale FE procedure into a code (cell level + structural level) seems still not fully practicable.

The present paper aims to bridge the gap of knowledge concerning the prediction of the ultimate behaviour of irregular masonry structures. To fulfil this target, a sufficiently simple (but reliable) model capable of giving estimates of failure load distributions and collapse mechanisms of full scale panels out-of-plane loaded is presented.

In the paper, considering that, for a random assemblage of blocks with variable dimensions, a full set of MC simulations is required to have an estimation of the averaged mechanical properties of the equivalent macroscopic material, a FE discretization of the REV is avoided, with the aim of drastically reducing the computational effort required to perform the numerical simulations. In particular, a compatible identification model already presented in [5] is considered here, assuming the REV constituted by a central block interconnected with its neighbors through mortar joints with zero thickness. In this framework masonry strength domains are obtained assuming blocks rigidinfinitely resistant and joints obeying a rigid-plastic behavior with associated flow rule. As a result, classic limit analysis theorems hold and simple linear programming tools may be used at a cell level to estimate, in a fast and reliable way, masonry macroscopic strength domains.

Limit analysis has been used by many authors in the recent past to estimate the load bearing capacity of masonry structures (e.g. Ferris and Tin Loi [20], Orduna and Lourenço [21]), even in combination with homogenization (de Buhan and de Felice [19], Milani et al. [22], Milani et al. [23]), because it requires only a reduced number of material parameters, providing interesting information at failure, such as for instance limit multipliers of loads, failure mechanisms and, at least on critical sections, the stress distribution.

Following the proposal for the in-plane case from [5], for joints a cohesive associated frictional behavior with limited tensile and compressive strength is assumed. Despite the fact that frictional phenomena may require the adoption of non-associated flow rules for the constituent materials (see for instance Begg and Fishwick [26], Ferris and Tin Loi [20], Orduna and Lourenço [21]), it has been shown that the assumption of associated plasticity (de Buhan and de Felice [19]) is able to provide reliable results, especially when failure mechanisms are mainly due to joints tensile cracking (e.g. [22]), as it occurs for out-of-plane actions.

In the model, the masonry skeleton is represented by a $3 \mathrm{D}$ discrete system of blocks interacting through interfaces (the mortar joints). Modeling the REV as a molecular skeleton allows to strongly reduce degrees of freedom and therefore permits to perform full Monte Carlo simulations in case of geometrical stochastic variability of the geometry. An automatic generation of REVs obtained assuming blocks with variable height and length following a predetermined random distribution is 
implemented. In this way, the statistical variability of the horizontal and vertical position of joints is automatically handled. This allows to consider several different REVs, which can be used repeatedly during Monte Carlo simulations. For a fixed geometry of the REV, a full description of the model is given considering a representative volume constituted by a generic block interconnected with its neighbors. A sub-class of possible elementary deformation modes (mimicking horizontal and vertical stretching, a pure shear deformation, pure bending and torsion along material axes) acting in the unit cell is a priori chosen in order to describe joints cracking under normal and tangential actions. Then, power dissipated in the discrete model is equated to that dissipated in a continuum macroscopic $2 \mathrm{D}$ equivalent plate. Such identification is based on a simple correspondence between motions in the 3D discrete model and the continuum. Since internal dissipation can take place only at the interface between blocks, a simple constrained minimization problem in a few variables is obtained. For a fixed REV geometry and for a fixed pattern, macroscopic masonry out-of-plane failure surfaces are numerically evaluated as a function of the macroscopic bending moments and torsion.

Due to the inexpensiveness of the approach proposed, large Monte Carlo simulations are repeated on the REV in order to obtain a large set of strength domains, each one depending on the geometry of the cell sampled. The potential advantage of the simple approach proposed with respect to traditional homogenization is therefore the possibility to repeat quickly a number of simulations with several different REV textures (generated randomly), thus allowing to obtain estimates of masonry strength distributions (mean values along different load directions, upper and lower bounds, standard deviation, etc.) quite efficiently.

Two cases are discussed: the first case is an elementary cell assembled with blocks with variable dimensions and without a preferential horizontal alignment, whereas the second case is a REV with a preferential horizontal alignment of the blocks (i.e. blocks disposed on the same course have the same height).

Afterwards, masonry homogenized failure surfaces are implemented in an upper bound FE limit analysis. To validate the model on small real scale structures, two examples of engineering interest, consisting respectively on a windowed panel constrained at three edges and a masonry rectangular wall simply supported at four edges and out-of-plane loaded by increasing pressures are critically discussed. Comparisons with expensive heterogeneous approaches are provided to validate the model proposed in terms of collapse load and failure mechanism. Both examples are meaningful because failure occurs for the formation of inclined yield lines, where plastic dissipation is due to a complex combination of vertical and horizontal bending moments (with a non-negligible contribution of torsion). Good agreement is found at structural level between the models, meaning 
that the homogenization presented represents a valuable tool for limit analysis of masonry structures constituted by random assemblages of blocks.

Finally, in order to show the capabilities of the approach proposed when dealing with full scale structures, a real structural example is presented, consisting in the analysis of a Romanesque masonry church façade located in Portugal and arranged in irregular texture. Comparisons with both FE heterogeneous approaches and "at hand" calculations show that reliable predictions of the load bearing capacity of real large scale façades may be obtained with a very limited computational effort.

\section{Masonry homogenized failure surfaces}

A simple model based on a correspondence between equivalent class of motions in a 3D discrete blocky system and a 2D continuum plate is presented in this section. The 3D blocky system and the 2D continuum are analyzed separately. Then, an equivalence procedure between the kinematic descriptors in the two systems is performed, equating power dissipated in the discrete and continuum model. It is worth noting that the formulation of the model does not impose a local field solution as, for instance, occurs using standard homogenization procedures, but imposes only a kinematic correspondence between motions. This assumption implies that the obtained solution is kinematically admissible and therefore represents an upper-bound of the actual solution.

\subsection{Basic assumptions: the random assemblage of blocks}

In order to take into account a large set of possible random blocks distributions, we consider two different blocks assemblages, as schematically depicted in Figure 1 and Figure 2. The first distribution, here denoted as Case I, concerns the random assemblage of blocks with variable height and length. The second case (Case II) is again a distribution with variable length and height of blocks, but with a horizontal alignment of blocks along the bed joint. An important sub-class of problems is obtained from Case II when the height is a deterministic variable (quasi periodic assemblage).

Let $C^{B}$ be the position of the centroid of the generic block $B_{i j}$ in the 3D Euclidean space. Let $\mathbf{e}_{1}$ $\mathbf{e}_{2}-\mathbf{e}_{3}$ indicate the global frame of reference with $\mathbf{e}_{1}$ indicating horizontal direction, $\mathbf{e}_{2}$ indicating vertical direction and $\mathbf{e}_{3}$ indicating the direction normal to the wall, Figure 3 and Figure 4. $i$ and $j$ indices assume integer values indicating the position of the blocks with respect to the central blocks. For instance, $i=1$ and $j=1$ indicate the first block positioned on the right with respect to the central 
block. Subsequent blocks are disposed with one interface common to the central block in counterclockwise order.

More in detail, the random REV is built block by block starting from the central block by introducing a random perturbation on horizontal and vertical position of blocks centroids neighboring central block and assuming that blocks length and height are stochastic variables with assigned distribution, i.e.:

$$
\begin{aligned}
& x_{B_{i, j}}=x_{B_{i-1}}+\delta_{1} \frac{L_{B_{i-1}}}{2}+\delta_{2} \frac{\tilde{L}_{B_{i}}}{2} \\
& y_{B_{i, j}}=y_{B_{i-1}}+\gamma_{1} \frac{H_{B_{i-1}}}{2}+\gamma_{2} \frac{\tilde{H}_{B_{i}}}{2}
\end{aligned}
$$

Where $\tilde{L}$ and $\tilde{H}$ are sampled values of blocks length and height from normal distribution with mean values $\bar{L}$ and $\bar{H}$ and standard deviations $\sigma_{L}$ and $\sigma_{H}$, respectively. The parameters $\delta_{1,2}$ and $\gamma_{1,2}$ suitably assume values equal to $-1,0,1$ following a counterclockwise disposition.

Depending on the choice of parameters $\delta_{1,2}$ and $\gamma_{1,2}$, two different classes of problems have been analyzed, namely a disposition with no alignment along the horizontal and vertical direction, as shown in Figure 2-a, or a preferential disposition preserving the bed joint direction, as depicted in Figure 2-b.

\section{$2.23 D$ blocky system: the heterogeneous model}

The 3D blocky system is represented by the random assemblage of infinitely resistant blocks with variable dimensions connected by joints reduced to interfaces with a rigid-plastic behavior (Figure 3). For a generic block $A$ in the REV, three centroid velocities $\mathbf{v}^{C_{A}}=\left[\begin{array}{lll}v_{x x}^{C_{A}} & v_{y y}^{C_{A}} & v_{z z}^{C_{A}}\end{array}\right]^{T}$ and three rotation rates $\boldsymbol{\Phi}^{A}=\left[\begin{array}{lll}\Phi_{x x}^{A} & \Phi_{y y}^{A} & \Phi_{z z}^{A}\end{array}\right]^{T}$ must be introduced. In order to evaluate power dissipated in the discrete system, it is necessary to take into consideration the interaction between two contiguous blocks, $A$ and $B$.

Let the point $P$ (local coordinates $\xi_{1}, \xi_{2}$ ) be a generic point on the interface $I$ between $A$ and $B$, as illustrated in Figure 5. Since $P \in I$ belongs respectively to $A$ and $B$ (where $I$ indicates the common interface between the two blocks), the following relations can be written:

$$
\begin{aligned}
& \mathbf{v}^{A}(P)=\mathbf{v}^{C^{A}}+\mathbf{M}\left(\boldsymbol{\Phi}^{A}\right)\left(P-\mathbf{C}^{A}\right) \\
& \mathbf{v}^{B}(P)=\mathbf{v}^{C^{B}}+\mathbf{M}\left(\boldsymbol{\Phi}^{B}\right)\left(P-\mathbf{C}^{B}\right)
\end{aligned}
$$

Here, $\mathbf{v}^{A}(P)\left(\mathbf{v}^{B}(P)\right)$ is the velocity of point $P$, which is considered belonging to block $A(B)$, and $\mathbf{M}(\mathbf{\Phi})$ is the following $3 \times 3$ skew matrix: 
$\mathbf{M}(\boldsymbol{\Phi})=\left[\begin{array}{ccc}0 & -\Phi_{z z} & \Phi_{y y} \\ \Phi_{z z} & 0 & -\Phi_{x x} \\ -\Phi_{y y} & \Phi_{x x} & 0\end{array}\right]$

In equation ( 2 ), the position of point $P$ is evaluated with respect to a local frame $\left(\xi_{1} \xi_{2}\right)$ with origin on the centroid on the interface, see Figure 5. Therefore, the jump of velocity $[\mathbf{v}(P)]$ between blocks $A$ and $B$ in a point $\xi \in I$ is expressed by:

$\lfloor\mathbf{v}(P)\rfloor=\mathbf{v}^{B}(P)-\mathbf{v}^{A}(P)=\mathbf{v}^{C^{A}}-\mathbf{v}^{C^{B}}+\mathbf{M}\left(\boldsymbol{\Phi}^{A}\right)\left(P-\mathbf{C}^{A}\right)-\mathbf{M}\left(\boldsymbol{\Phi}^{B}\right)\left(P-\mathbf{C}^{B}\right)$

and the power dissipated at the interface $I$ can be written as:

$\pi=\int_{I}\left[\mathbf{t}^{A}(P) \cdot \mathbf{v}^{A}(P)+\mathbf{t}^{B}(P) \cdot \mathbf{v}^{B}(P)\right] d S=\int_{I} \mathbf{t}^{A}(P) \cdot[\mathbf{v}(P)] d S$

where $\mathbf{t}^{A}(P)=\left[\begin{array}{lll}\tau_{13}(P) & \tau_{23}(P) & \sigma_{33}(P)\end{array}\right]^{T}\left(\mathbf{t}^{B}(P)\right.$ is the stress vector acting at $\xi$ on block $A(B)$, see Figure 5, with $\left.\mathbf{t}^{A}(P)=-\mathbf{t}^{B}(P)\right)$.

\subsection{Continuous 2D plate model}

For the general problem involving both in- and out-of-plane actions, a standard 2D Cauchy continuum, identified by its middle plane $S$ of normal $\mathbf{e}_{3}$ (Figure 4 ), is assumed as an equivalent plate homogenized model. The velocity field of a point $\mathbf{P}$ (coordinates $\left[\begin{array}{lll}x_{1}^{P} & x_{2}^{P} & x_{3}^{P}\end{array}\right]$ ) belonging to the equivalent continuum plate is given by fields $\mathbf{w}(\mathbf{x})$ (components $w_{1}, w_{2}$ and $\left.w_{3}\right)$ and $\mathbf{\Psi}(\mathbf{x})$ (components $\Psi_{1}$ and $\Psi_{2}$ ), representing respectively the velocity and rotation rates of the plate in correspondence of the point $\mathbf{x}=\left[\begin{array}{lll}x_{1}^{P} & x_{2}^{P} & 0\end{array}\right]$ laying in the middle plane of the plate.

The stored energy density in the equivalent plate model is:

$$
\pi=\left[\begin{array}{lll}
N_{11} & N_{12} & N_{22}
\end{array}\right]\left[\begin{array}{c}
\dot{E}_{11} \\
\dot{E}_{12}+\dot{E}_{21} \\
\dot{E}_{22}
\end{array}\right]+\left[\begin{array}{ll}
T_{13} & T_{23}
\end{array}\right]\left[\begin{array}{c}
\dot{\gamma}_{13} \\
\dot{\gamma}_{23}
\end{array}\right]+\left[\begin{array}{lll}
M_{11} & M_{12} & M_{22}
\end{array}\right]\left[\begin{array}{c}
\dot{\chi}_{11} \\
\dot{\chi}_{12}+\dot{\chi}_{21} \\
\dot{\chi}_{22}
\end{array}\right]
$$

Where the symbols in equation ( 6 ) have the following meaning:

$-\dot{\mathbf{E}}=\left[\begin{array}{c}\dot{E}_{11} \\ \dot{E}_{12}+\dot{E}_{21} \\ \dot{E}_{22}\end{array}\right]=\frac{1}{t} \int_{-t / 2}^{t / 2}\left[\begin{array}{c}\partial w_{1} / \partial x_{1}-x_{3} \partial \Psi_{1} / \partial x_{1} \\ \partial w_{2} / \partial x_{1}+\partial w_{1} / \partial x_{2}-x_{3}\left(\partial \Psi_{1} / \partial x_{2}+\partial \Psi_{2} / \partial x_{1}\right) \\ \partial w_{2} / \partial x_{2}-x_{3} \partial \Psi_{2} / \partial x_{2}\end{array}\right] d x_{3}$ is the in-plane strain rate vector, assuming $t$ as the masonry thickness, $\mathbf{w}=\left[\begin{array}{lll}w_{1} & w_{2} & w_{3}\end{array}\right]^{T}$ the velocity field in the continuous model (local frame of reference) and $\boldsymbol{\Psi}=\left[\begin{array}{lll}\Psi_{1} & \Psi_{2} & \Psi_{3}\end{array}\right]^{T}$ the rotation rate field; 
$-\dot{\gamma}=\left[\begin{array}{l}\dot{\gamma}_{13} \\ \dot{\gamma}_{23}\end{array}\right]=\frac{1}{t} \int_{-t / 2}^{t / 2}\left[\begin{array}{l}\partial w_{3} / \partial x_{1}+\partial w_{1} / \partial x_{3} \\ \partial w_{3} / \partial x_{2}+\partial w_{2} / \partial x_{3}\end{array}\right] d x_{3}$ is the shear strain rate vector;

$-\dot{\chi}=\left[\begin{array}{c}\dot{\chi}_{11} \\ \dot{\chi}_{12}+\dot{\chi}_{21} \\ \dot{\chi}_{22}\end{array}\right]=\frac{1}{t} \int_{-t / 2}^{t / 2}\left[\begin{array}{c}\partial \Psi_{1} / \partial x_{1} \\ \partial \Psi_{2} / \partial x_{1}+\partial \Psi_{1} / \partial x_{2} \\ \partial \Psi_{2} / \partial x_{2}\end{array}\right] d x_{3}$ is the curvature rate vector;

- $\mathbf{M}=\left[\begin{array}{lll}M_{11} & M_{12} & M_{22}\end{array}\right]^{T}$ is the homogenized moments vector, with $M_{11}$ and $M_{22}$ indicating bending and $M_{12}$ torsion;

- $\mathbf{T}=\left[\begin{array}{ll}T_{13} & T_{23}\end{array}\right]^{T}$ is the homogenized out-of-plane shear vector;

- $\mathbf{N}=\left[\begin{array}{lll}N_{11} & N_{12} & N_{22}\end{array}\right]^{T}$ is the homogenized membrane actions vector.

It is worth noting here that the out-of-plane case here treated represents a full Reissner-Mindlin homogenization approach and it is therefore suitable for the analysis of both thin and thick walls and also may be generalized to multi-leaf panels. However, for the simulations reported hereafter, we assume $\dot{\gamma}=\mathbf{0}$ (Kirchhoff-Love hypothesis), because of the moderate thickness of the panels investigated.

\subsection{Identification (power equivalence)}

In order to substitute the heterogeneous material with the homogeneous equivalent $2 \mathrm{D}$ model, see Figure 6, a simple compatible identification model is proposed, assuming that the power dissipated by blocks assemblage, equation ( 5 ), is equal to the power dissipated by the equivalent model, equation ( 6 ). For this purpose, fields $\mathbf{w}(\mathbf{x})$ and $\Psi(\mathbf{x})$ are a priori chosen as a combination of elementary deformations in the unit cell, corresponding to actual failure mechanisms occurring in presence of infinitely resistant blocks with weak joints reduced to interfaces. From a practical point of view, fields $\mathbf{w}(\mathbf{x})$ and $\Psi(\mathbf{x})$, corresponding to each sub-class of regular motions, are obtained assuming alternatively one component of vector $\dot{\mathbf{E}}, \dot{\gamma}$ or $\dot{\chi}$ unitary and setting all the other components equal to zero, subsequently choosing the most simple polynomial expressions for $\mathbf{w}(\mathbf{x})$ and $\Psi(\mathbf{x})$ that comply with equation ( 6 ). Once fields $\mathbf{w}(\mathbf{x})$ and $\boldsymbol{\Psi}(\mathbf{x})$ are known, rotation rates and velocities of each block belonging to the REV in the heterogeneous model are determined assuming point $\mathbf{x}$ as the centroid of the block under consideration.

For instance, when only $\dot{\chi}_{11} \neq 0$ is applied on the REV, a choice for $\mathbf{w}(\mathbf{x})$ and $\Psi(\mathbf{x})$ fields is: $\Psi_{1}=\dot{\chi}_{11} x_{1}$ 
$w_{1}=\dot{\chi}_{11} x_{1} x_{3}$

$w_{2}=0$

$w_{3}=-\dot{\chi}_{11} x_{1}^{2} / 2$

Equation ( 7 ) allows for the direct determination of velocities and rotations of each block, provided that the coordinates of the respective centroid are introduced in ( 7 ).

Since a Kirchhoff-Love plate hypothesis is adopted, we assume $\dot{\gamma}=\mathbf{0}$, considering all the possible remaining combinations of the in-plane and out-of-plane deformation rate tensors $\dot{\mathbf{E}}$ and $\dot{\chi}$.

In particular, in Figure 7-a the effect on a randomly assembled REV of a homogeneous deformation $\dot{E}_{11} \neq 0$ with all the other strain measures set to zero is represented. Similarly to the deterministic case, it is interesting to note that both head and bed joints contribute to the dissipation induced by this deformation. Figure 7-b shows the effect on the brickwork of a homogeneous deformation in which $\dot{E}_{12}+\dot{E}_{21} \neq 0$ (shear mode) and all the other strain measures are set to zero, whereas Figure 7-c shows the effect of a vertical in-plane homogeneous deformation $\dot{E}_{22} \neq 0$. In this latter case, differently from a regular assemblage of blocks, for an irregular texture both head and bed joints may contribute to the overall strength of the continuous material, resulting in an overall strength greater than that of the horizontal joint in pure tension. On the contrary, when dealing with quasiperiodic masonry (i.e. with a horizontal alignment along bed joints), failure due to a macroscopic deformation $\dot{E}_{22} \neq 0$ involves only the bed joints, leading to the same behavior observed for the deterministic running bond case. Similar considerations may be repeated for the out-of-plane deformation modes, depicted from Figure 7-d to -f. Analogously to the in-plane case, in fact, the irregular disposition of blocks generates bending moment and torsion both in the head and bed joints when a curvature rate $\dot{\chi}_{22} \neq 0$ is applied on the REV. As a consequence, also for the out-ofplane action, a greater resistance with respect to the strength of the bed joint is expected for the homogenized material in vertical bending. On the other hand, in horizontal bending, the maximum strength that can be achieved is that of the deterministic running bond case, which produces a symmetrical failure mechanism involving bed joint torsional resistance for a length equal to one half of the length of the central block.

\subsection{Stochastic masonry failure surfaces}

In this section, following the original formulation provided by Suquet [27], a general numerical procedure for obtaining macroscopic masonry failure surfaces is presented. Both static and kinematic theorems of limit analysis can be used for this purpose. In this framework, it is worth noting that several different models have been presented in the literature for the evaluation of in- 
plane (e.g. Milani et al. [22]) and out-of-plane masonry failure surfaces (Sab [28], Cecchi and Milani [24]).

One of the basic assumptions of this approach is the utilization of associated flow rules for the constituent materials. Nevertheless, it is worth mentioning that sliding occurs in mortar joints with almost zero dilatancy, with typical non-associativity. The violation of one of the hypotheses of classic limit analysis [20][21], implies that the uniqueness of the ultimate load may be lost and a multiplicity of solutions can exist, see Begg and Fishwick [26]. On the contrary, the assumption of associated flow rules ensures the uniqueness of the ultimate load factor and leads to simple optimization problems which can be handled easily with linear programming (LP) packages.

A failure criterion $\phi=\phi(\boldsymbol{\sigma})$ for the joints must be incorporated. The basic failure modes for masonry walls with weak mortar are a mixing of sliding along the joints (a), cracking of the joints (b) and compressive masonry crushing (c). These modes can be well reproduced adopting a MohrCoulomb failure criterion combined with a tension cut-off and a cap in compression, see Figure 8, as suggested by Lourenço and Rots [1].

Aiming at treating the problem in the framework of linear programming, within each interface $I$ of area $A^{I}$, a piecewise linear approximation of the failure surface $\phi=\phi(\boldsymbol{\sigma})$ is adopted, constituted by $n_{\text {lin }}$ planes of equation $\mathbf{A}_{i}^{I^{T}} \boldsymbol{\sigma}=c_{i}^{I} \quad 1 \leq i \leq n_{\text {lin }}$, where $\boldsymbol{\sigma}=\left[\begin{array}{lll}\sigma_{33} & \tau_{13} & \tau_{23}\end{array}\right], \sigma_{33}$ is the normal stress on the interface and $\tau_{13}$ and $\tau_{23}$ are tangential stresses along two assigned perpendicular directions $\left(A_{i}^{1 I} \sigma_{33}+A_{i}^{2 I} \tau_{13}+A_{i}^{3 I} \tau_{23}=c_{i}^{I}\right.$ is the $i$-th linearization plane of the interface $I$, with $\left.\mathbf{A}_{i}^{I^{T}}=\left[\begin{array}{lll}A_{i}^{1 I} & A_{i}^{2 I} & A_{i}^{3 I}\end{array}\right]\right)$, Figure 5 and Figure 8.

The jump of velocity on interfaces varies linearly in the discrete model, equation ( 4 ). Thus, for each interface, only $3 \cdot n_{\text {lin }}$ independent plastic multiplier rates have to be introduced as optimization variables. Furthermore, for each interface $I$ between contiguous blocks, the following equality constraints between plastic multiplier rates $\dot{\lambda}_{i}^{I}\left(\xi_{1}, \xi_{2}\right)$ and jump of velocity $\left[\mathbf{v}\left(\xi_{1}, \xi_{2}\right)\right]$ on the interface must be imposed:

$$
\left[\mathbf{v}\left(\xi_{1}, \xi_{2}\right)\right]=\sum_{i=1}^{n_{\text {lin }}} \dot{\lambda}_{i}^{I}\left(\xi_{1}, \xi_{2}\right) \frac{\partial \phi}{\partial \boldsymbol{\sigma}}
$$

where:

- $\xi=\left(\xi_{1}, \xi_{2}\right)$ is a local frame of reference laying on the interface plane and with axis $\xi_{3}$ orthogonal to the interface plane, Figure 8; 
- $\left[\mathbf{v}\left(\xi_{1}, \xi_{2}\right)\right]=\left[\begin{array}{llll}\Delta v_{33} & \Delta v_{13} & \Delta v_{23}\end{array}\right]^{T}$ is the jump of velocity field (linear in $\left.\left(\xi_{1}, \xi_{2}\right)\right)$ on the $I-$ th interface and $\Delta v_{i j}$ corresponds to the jump along the direction $j$.

- $\dot{\lambda}_{i}^{I}\left(\xi_{1}, \xi_{2}\right)$ is the $i$-th plastic multiplier rate field (linear in $\left(\xi_{1}, \xi_{2}\right)$ ) of the interface $I$, associated to the $i$-th linearization plane of the failure surface.

It is worth noting that, in order to satisfy equation ( 8 ) for each point of the interface $I$, nine equality constraints for each interface have to be imposed, which corresponds to evaluating ( 8 ) in three different positions $P_{k}=\left(\xi_{1}^{P_{k}}, \xi_{2}^{P_{k}}\right)$ on the interface $I$ as follows:

$$
\left[\mathbf{v}\left(\xi_{1}^{P_{k}}, \xi_{2}^{P_{k}}\right)\right]=\sum_{i=1}^{n_{\text {lin }}} \dot{\lambda}_{i}^{I}\left(\xi_{1}^{P_{k}}, \xi_{2}^{P_{k}}\right) \frac{\partial \phi}{\partial \boldsymbol{\sigma}} \quad k=1,2,3
$$

Here, $\dot{\lambda}_{i}^{I}\left(\xi_{1}^{P_{k}}, \xi_{2}^{P_{k}}\right)$ is the is $i$-th plastic multiplier rate of the interface $I$ corresponding to $P_{k}=\left(\xi_{1}^{P_{k}}, \xi_{2}^{P_{k}}\right)$.

From the previous equations, the internal power dissipated on the $I$-th interface can be written as:

$$
\pi_{\mathrm{int}}^{I}=\int_{A^{I}}[\mathbf{v}]^{T} \boldsymbol{\sigma} d A^{I}=\int_{A^{I}} \sum_{i=1}^{n_{\text {in }}} \dot{\lambda}_{i}^{I}\left(\xi_{1}, \xi_{2}\right)\left[\frac{\partial \phi}{\partial \boldsymbol{\sigma}}\right]^{T} \boldsymbol{\sigma} d A^{I}=\frac{1}{4} \sum_{i=1}^{n_{\text {lin }}} c_{i}^{I} \sum_{k=1}^{4} \dot{\lambda}_{i}^{I}\left(\xi_{1}^{P_{k}}, \xi_{2}^{P_{k}}\right) A^{I}
$$

It is worth noting that in equation ( 10 ) only three of the four plastic multipliers are linearly independent, whereas $k=4$ depends linearly on $k=1,2,3$ (the plastic multiplier field is linear on the interface).

The external power dissipated can be written as $\pi_{e x t}=\left(\boldsymbol{\Sigma}_{0}^{T}+\lambda \boldsymbol{\Sigma}_{1}^{T}\right) \mathbf{D}$, where $\boldsymbol{\Sigma}_{0}$ is the vector of dead loads, $\lambda$ is the load multiplier, $\boldsymbol{\Sigma}_{1}^{T}$ is the unitary vector of loads dependent on the load multiplier (i.e. the optimization direction in the space of macroscopic stresses) and $\mathbf{D}$ is the vector of macroscopic kinematic descriptors. D collects in-plane deformation rates $\left(\dot{E}_{11} 0.5\left(\dot{E}_{12}+\dot{E}_{21}\right) \dot{E}_{22}\right)$, Kirchhoff-Love out-of-plane curvature rates $\left(\dot{\chi}_{11}\left(\dot{\chi}_{12}+\dot{\chi}_{21}\right) / 2 \dot{\chi}_{22}\right)$ and out-of-plane shear deformation rates $\left(\dot{\gamma}_{13}\right.$ and $\left.\dot{\gamma}_{23}\right)$. As the amplitude of the failure mechanism is arbitrary, a further normalization condition $\boldsymbol{\Sigma}_{1}^{T} \mathbf{D}=1$ is usually introduced. Hence, the external power becomes linear in $\mathbf{D}$ and $\lambda$ and can be written as follows $\pi_{e x t}=\Sigma_{0}^{T} \mathbf{D}+\lambda$.

From the above considerations, the optimization variables necessary to determine masonry homogenized strength domain are respectively the vector of macroscopic kinematic descriptors D and the vector of assembled plastic multiplier rates $\dot{\lambda}^{I}$ at each mortar interface.

From equations ( 7 ) and ( 4 ), a further set of linear equality constraints has to be imposed at each interface $I$, involving vector $\mathbf{D}$ and jump of displacements field $\left[\mathbf{v}\left(\xi_{1}, \boldsymbol{\xi}_{2}\right)\right]$ : 
where $\mathbf{G}^{I}\left(\xi_{1}, \xi_{2}\right)$ is a $3 \times 8$ matrix that depends only on the geometry of the interface under consideration. It is interesting to notice that, from equations ( 9 ) and ( 11 ), the jump of velocities $\left[\mathbf{v}\left(\xi_{1}, \xi_{2}\right)\right]$ does not enter as optimization variable in the optimization problem at a cell level, being $\mathbf{G}^{I}\left(P_{k}\right) \mathbf{D}=\left[\mathbf{v}\left(P_{k}\right)\right]=\sum_{i=1}^{n_{i n}} \dot{\lambda}_{i}^{I}\left(\xi_{1}^{P_{k}}, \xi_{2}^{P_{k}}\right) \frac{\partial \phi}{\partial \boldsymbol{\sigma}} \quad P_{k} \in I$. In particular, from equations ( 9 ), , 10 ), ( 11 ) and from the kinematic formulation of limit analysis, the following constrained minimization problem has to be solved to obtain masonry failure surfaces:

$$
\left\{\begin{array}{c}
\lambda=\min _{\hat{\mathbf{x}}=\left[\mathbf{D}, \lambda_{i}^{I}\left(P_{k}\right)\right]} \sum_{I=1}^{n^{I}} \pi_{\mathrm{int}}^{I}-\boldsymbol{\Sigma}_{0}^{T} \mathbf{D} \\
\boldsymbol{\Sigma}_{1}^{T} \mathbf{D}=1 \\
\mathbf{G}^{I}\left(P_{k}\right) \mathbf{D}=\left[\mathbf{v}\left(P_{k}\right)\right]=\sum_{i=1}^{n_{\text {in }}} \lambda_{i}^{I}\left(\xi_{1}^{P_{k}}, \xi_{2}^{P_{k}}\right) \frac{\partial \phi}{\partial \boldsymbol{\sigma}} \quad P_{k} \in I
\end{array}\right.
$$

Here, $n^{I}$ is the total number of interfaces considered and $\hat{\mathbf{x}}$ is the vector of total optimization unknowns. The linear programming problem ( 12 ) involves a relatively small number of optimization variables and therefore can be solved by means of simplex or interior point methods (vector $\hat{\mathbf{x}}$ of global unknowns collects only $3 \cdot n_{\text {lin }} \cdot n^{I}$ plastic multiplier rates and 8 macroscopic kinematic variables D). When a Kirchhoff-Love plate hypothesis is assumed (as in the present paper), $\mathbf{D}$ is a vector of length 6 collecting in-plane macroscopic deformation rates $\left(\dot{E}_{11}\right.$, $0.5\left(\dot{E}_{12}+\dot{E}_{21}\right)$ and $\left.\dot{E}_{22}\right)$ and curvature rates $\left(\dot{\chi}_{11}, 0.5\left(\dot{\chi}_{12}+\dot{\chi}_{21}\right)\right.$ and $\left.\dot{\chi}_{22}\right)$. Usually, macroscopic strength domains are presented in numerical results as surface sections in the space of bending moments and torsion $\left(M_{11}, M_{12}\right.$ and $\left.M_{22}\right)$, where membrane actions are kept constant ( $N_{11}=N_{12}=0$ and $N_{22}=k$ ). Obviously, the optimal value $\lambda$ obtained from ( 12 ) represents only a point on $\hat{\Phi}$, i.e. the intersection between surface $\hat{\Phi}$ and the direction unit vector $\boldsymbol{\Sigma}_{1}$, see also Figure 9. Consequently, in order to obtain a reliable linear approximation of $\hat{\Phi}$ by means of Delaunay tessellations, the linear programming problem ( 12 ) has to be solved several times, each problem corresponding to a different choice for $\boldsymbol{\Sigma}_{1}$ direction.

When dealing with out-of-plane actions and differently to the in-plane case [5], several optimization variables must be introduced in the process of the linearization of the failure surfaces of the constituent materials. Recent trends in limit analysis have demonstrated that the linearization of the strength domain can be circumvented using conic programming (e.g. Makrodimopoulos and Martin [29], Krabbenhoft et al. [30]). This tool is more powerful with respect to LP and could lead to less 
expensive processing times, especially when large sets of Monte Carlo simulations have to be performed. Nonetheless, here classic interior point LP routines are used, because the processing time needed is still acceptable.

\subsection{Applications at a cell level}

Two meaningful applications at a cell level are here analyzed with the model proposed, the first consisting in a random assemblage of blocks without preferential lines in their disposition (Case I) and the second relying in texture obtained with blocks of variable size but with horizontal joints continuity preserved (Case II). In both cases, large scale Monte Carlo simulations are performed $(10,000)$. Two sections of the 3D out-of-plane failure surfaces are represented, namely vertical bending $\left(M_{11}\right)$ - horizontal bending $\left(M_{22}\right)$ and vertical bending $\left(M_{11}\right)$-torsion $\left(M_{12}\right)$ sections. At a fixed REV geometry (corresponding to a single Monte Carlo simulation), failure surface sections are obtained with the model proposed investigating 40 different $\psi$ angles equally stepped, thus requiring to solve iteratively a total of 1,200,000 optimization problems ( 12 ) for each section.

In both cases, due to the relatively limited number of variables involved in the simulations, the computational effort required to perform such large scale Monte Carlo simulations did not exceed 5,000 seconds for each set of simulations, meaning that the procedure proposed may be a valuable numerical tool for practitioners interested in a reliable and fast estimation of collapse load distributions of random blocks assemblages. Due to the acceptable computational effort required, a meaningful comparison between results obtained supposing joints obeying a classic Mohr-Coulomb and a Lourenço-Rots failure criterion [1] is also reported.

\subsubsection{Case I: random assemblage through blocks with variable dimensions}

A masonry wall arranged with rectangular blocks disposed in irregular texture as in Case I and mortar joints reduced to interfaces is considered. For the blocks, a stochastic normal distribution for length $\mathrm{L}$ and height $\mathrm{H}$, with mean values equal to 300 and $200 \mathrm{~mm}$ respectively for $\mathrm{L}$ and $\mathrm{H}$ (thickness $\mathrm{t}=200 \mathrm{~mm}$ ). Standard deviations are set equal to 80 and $60 \mathrm{~mm}$ respectively for length and height. Mechanical properties at failure adopted for the constituent materials are summarized in Table I. In order to show the influence of the failure surface adopted for the interfaces on the overall strength of the blocks assemblage, both a classic Mohr-Coulomb and a linearized Lourenço and Rots [1] failure criterion are assumed for joints.

In Figure 10-a, $\mathbf{M}_{11}-\mathrm{M}_{22}$ masonry strength domain sections obtained with the model proposed sampling a total number of 10,000 different REVs are depicted. Furthermore, in Figure 10 from $-\mathrm{b}$ to $-\mathrm{d}$, empirical cumulative distribution functions (ECDF) for the failure multiplier $\lambda$ at 3 assigned 
$\psi$ angles $\left(0^{\circ}, 45^{\circ}\right.$ and $\left.90^{\circ}\right)$ are reported, with the corresponding evaluation of mean and standard deviation values. The same results are repeated in Figure 11 for $\mathrm{M}_{11}-\mathrm{M}_{12}$ sections.

Here, it is worth noting that distributions with $\psi$ angles respectively equal to $180^{\circ}, 225^{\circ}$ and $270^{\circ}$ are almost identical (same absolute mean value and standard deviation) to those found for $\psi$ equal to $0^{\circ}, 45^{\circ}$ and $90^{\circ}$. For this reason, they are not reported in the figure for the sake of conciseness.

The same simulations are replicated in Figure 12 and Figure 13 respectively for $\mathbf{M}_{11}-\mathbf{M}_{22}$ and $\mathbf{M}_{11^{-}}$ $\mathrm{M}_{12}$ sections, but in this case assuming for joints a classic Mohr-Coulomb failure criterion with the same friction angle and the same tensile strength (cohesion is therefore equal to $c=f_{t} \tan (\Phi)$ ). In Figure 12, masonry strength in horizontal bending when a deterministic running bond texture is assumed is also represented (yellow dot). It is particularly interesting to notice, as already pointed out, that this strength value is an upper-bound for the stochastic distribution of blocks considered. For the sake of completeness, in Figure 14 some typical failure mechanisms obtained at fixed $\psi$ angle are reported for some sampled REV dispositions (Lourenço and Rots [1] failure criterion). From an overall analysis of simulations results, it is worth noting that the minimum envelope of the resultant homogenized failure surfaces (defined as the smallest possible strength exhibited by the random assemblage at a fixed $\psi$ angle) is isotropic and obviously corresponds to a homogenized failure surface obtained supposing masonry constituted by mortar joints reduced to interfaces interconnected by blocks disposed in stack bond texture (i.e. with both vertical and horizontal alignment). This aspect is particularly clear when a Mohr-Coulomb failure criterion is adopted for joints.

Finally, the differences between failure surfaces sections obtained assuming either a Lourenço-Rots or a Mohr-Coulomb strength domain are worth noting, especially when $\mathbf{M}_{11}-\mathbf{M}_{12}$ sections are considered (compare, for instance, Figure 11 and Figure 13). In fact, when dealing with a LourençoRots failure criterion, Figure 11, the lower bound strength domain section is constituted, in the range $0 \leq \psi \leq 90^{\circ}$ by a vertical and an inclined segment. The vertical segment corresponds to a failure in horizontal bending due to the activation of plastic multiplier referred to the tensile strength $f_{t}$, with corresponding bending moment at failure equal to $f_{t} t^{2} / 2$. On the other hand, the inclined segment corresponds to a frictional failure exactly equal to that exhibited by the elementary cell assuming a pure Mohr-Coulomb failure criterion, see Figure 13 (remembering that cohesions in the models are diffent, being in the Mohr-Coulomb failure criterion $c=f_{t} \tan (\Phi)$ ). As it is possible to notice, in Figure 13, the lower bound envelope in the range $0 \leq \psi \leq 90^{\circ}$ is constituted only by an inclined segment, which corresponds to an integration along the thickness of the ultimate strength domain $\sigma_{12}=\tan \Phi \sigma_{11}+c$. Such ultimate resistance is related to a mechanism exhibiting a 
combined failure in torsion and horizontal flexion. Obviously, it is analogous to that obtained assuming a Lourenço-Rots strength domain until, in the framework of the upper bound theorem of limit analysis, a flexural failure in horizontal bending provides a limit multiplier lower to that associated to the frictional failure. On the other hand, it is worth noting that a Mohr Coulomb failure criterion corresponds exactly to a Lourenço-Rots strength domain when $f_{t} \rightarrow \infty$ and $f_{c} \rightarrow \infty$.

Furthermore, considering the $\mathrm{M}_{11}-\mathrm{M}_{22}$ failure sections, it is interesting to notice that an overstrength is possible, with respect to the deterministic stack and running bond case of the elementary cell in vertical bending (differently to the second configuration, which will be analyzed further). This behavior is a consequence of the possible misalignment of the bricks along the vertical direction (missing the continuity of the bed joints), which allows the vertical interfaces to contribute with their torsional strength to the overall resistance of the REV.

It is stressed that failure surfaces numerically obtained are collected in a suitable database, which will be implement successively at a structural level for upper bound limit analyses of entire masonry walls out-of-plane loaded.

\subsubsection{Case II: random assemblage with preferential horizontal disposition}

An assemblage of blocks with preferential horizontal disposition is considered as a second example. The same stochastic distribution of the previous case is assumed for the block, but maintaining here a fixed horizontal alignment and staggering blocks along two contiguous rows. Mechanical properties at failure adopted for the constituent materials are the same of the previous example, see Table I. In particular, a Lourenço-Rots [1] and a classic Mohr-Coulomb are assumed for joints (with the same friction angle and cohesion).

In Figure 15-a, $\mathrm{M}_{11}-\mathrm{M}_{22}$ masonry strength domain sections obtained with a Lourenço-Rots [1] failure criterion for joints and resulting from a large scale Monte Carlo set of simulations is shown. In Figure 15 from $-\mathrm{b}$ to $-\mathrm{d}$, empirical cumulative distribution functions (ECDF) for the failure multiplier $\lambda$ at assigned $\psi$ angles have been reported, with the corresponding evaluation of mean and standard deviation values. Results are replicated in Figure 16 for $\mathrm{M}_{11}-\mathrm{M}_{12}$ failure surface sections. As in the previous case, distributions with $\psi$ angles respectively equal to $180^{\circ}, 225^{\circ}$ and $270^{\circ}$ are almost identical (same absolute mean value and standard deviation) to those found for $\psi$ equal to $0^{\circ}, 45^{\circ}$ and $90^{\circ}$. For this reason, they are not reported for the sake of conciseness.

The same simulations are repeated assuming for joints a Mohr-Coulomb failure criterion in Figure 17 and Figure 18 respectively for $\mathrm{M}_{11}-\mathrm{M}_{22}$ and $\mathrm{M}_{11}-\mathrm{M}_{12}$ sections. When dealing with the Mohr- 
Coulomb failure criterion, the same friction angle and tensile strength (cohesion $c$ equal to $f_{t} \tan (\Phi)$ ) used to perform simulations reported in Figure 15 are adopted.

For the sake of completeness, in Figure 19 some typical failure mechanisms obtained at fixed $\psi$ and $\vartheta$ angles are reported for some sampled REV dispositions. The same considerations done in the previous examples can be repeated here for the second disposition. Nevertheless, due to the horizontal alignment of the blocks allowing a continuous bed joint, there is no variability of the vertical bending strength for all the REVs inspected, which results deterministically equal to the bending strength of the horizontal joint $\left(f_{t} t^{2} / 2\right)$. In fact, elementary cell always fails along the preferential plane of weakness formed by the bed joint, leading to a homogenized strength equal to that of the bed joint.

Finally, from an overall analysis of Figure 17 and Figure 18, it is worth underlining that the upper bound failure envelopes found in the Mohr-Coulomb case correspond exactly to the homogenized failure surface sections obtained in the case of a deterministic running bond disposition (the reader is referred, for instance to [28] for a detailed comparison of results found in the present simulations). This remark is expected in light of the consideration that the second disposition is a running bond one, with stochastic misalignment along two contiguous horizontal rows. The upper bound strength exhibited by such pattern is therefore represented by the deterministic running bond cell, where the torque contribution of the bed joint to both $\mathrm{M}_{11}$ and $\mathrm{M}_{12}$ results from integration of the tangential actions on the bed joint over the maximum possible length, corresponding to the semi-length of the brick. All other intermediate situations can only reduce corresponding macroscopic values of both $\mathrm{M}_{11}$ and $\mathrm{M}_{12}$.

\section{Structural level implementation}

A 2D Reissner-Mindlin FE kinematic limit analysis model is used for the stochastic structural analysis at collapse of masonry walls out-of-plane loaded. The model can be indifferently used assuming a homogenized failure surface for masonry and in the framework of a heterogeneous approach. Nevertheless, when dealing with the homogenized approach, since a Kirchhoff-Love plate hypothesis is assumed at a cell level, the out-of-plane shear strength resistance is infinite. On the contrary, in the heterogeneous approach, a limited strength equal to that of the mortar joints is assumed, otherwise the formation of inclined yield lines is not possible and an infinite value of the failure multiplier can be found. It is stressed that, due to the limited thickness of the panels investigated, the models give comparable results and the error introduced through this simplification is not relevant. 
The formulation is based on a triangular discretization of 2D domains and on the introduction of discontinuities of the velocity field along the edges of adjacent triangles, see Figure 20. Triangles are supposed rigid infinitely resistant and plastic dissipation is allowed only at the interfaces between contiguous elements. It has been shown [23][24] that this simplification allows to study large scale structural problems with a very limited computational effort (thus allowing to perform expensive Monte Carlo simulations at a relatively low cost), but providing good estimates of collapse loads and failure mechanisms.

When dealing with a heterogeneous approach, bricks and joints are modeled separately at a structural level. Two different modeling strategies can be followed: the first is to reduce joints to interfaces, thus concentrating all the non-linearity on a segment representing mortar, whereas the second is to consider joint actual thickness during the discretization into Finite Elements. The first approach is widely used in the technical literature (e.g. [1][3][4][19][20]) with the aim of limiting the computational time need to solve even small scale panels. Obviously, such simplification is sufficiently accurate only when the thickness of the joints is small with respect to brick dimensions. Let us consider a triangular element $E$ representing a brick, a mortar or a homogenized material portion. Within $E$, a linear interpolation of the velocity field is assumed. Thus, one out-of-plane velocity unknown $w_{3 i}^{E}$ per node $i$ is introduced, Figure 20. Due to the linear interpolation of the velocity field inside each element, plastic dissipation can occur at the interface between adjoining elements due to the combined action of bending moment, torsion and out-of-plane shear. Differently from a well known elastic FE discretization, several nodes may share the same coordinate, being each node associated with only one element. In this way, at each interface between adjacent triangles, possible jumps of velocities can occur.

At each interface $M$ and $N$, both constant bending rotation rates $\dot{\omega}_{N-M}^{n n}$ and a torsional rotation rates $\dot{\omega}_{N-M}^{n t}$ can occur. Furthermore, an out-of-plane jump of velocities $\left[w_{3}\right]$ which varies linearly along the interface is also present. $\dot{\omega}_{N-M}^{n n}, \dot{\omega}_{N-M}^{n t}$ and $\left[w_{3}\right]$ can be easily evaluated making use of the following linear relation between nodal velocities of adjacent elements $M$ and $N$ (see Figure 20):

$$
\begin{aligned}
& \dot{\boldsymbol{\omega}}_{N}=\mathbf{B}_{N} \mathbf{w}_{3}^{N} \\
& \dot{\omega}_{N-M}^{n t}=\frac{w_{3 j}^{M}-w_{3 i}^{M}}{\Gamma_{I}}-\frac{w_{3 j}^{N}-w_{3 k}^{N}}{\Gamma_{I}} \\
& {\left[w_{3}\right]=\left(w_{3 j}^{M}-w_{3 j}^{N}\right)+\frac{x_{I}}{\Gamma_{I}}\left[\left(w_{3 i}^{M}-w_{3 k}^{N}\right)-\left(w_{3 j}^{M}-w_{3 j}^{N}\right)\right] \quad x_{I} \in\left[\begin{array}{ll}
0 & \Gamma_{I}
\end{array}\right]}
\end{aligned}
$$

Where: 
$-\mathbf{w}_{3}^{N}=\left[\begin{array}{lll}w_{3 i}^{N} & w_{3 j}^{N} & w_{3 k}^{N}\end{array}\right]^{T}$

$-\mathbf{B}_{N}=\frac{1}{2 A_{N}}\left[\begin{array}{lll}\frac{b_{i} b_{i}+c_{i} c_{i}}{l_{i}} & \frac{b_{i} b_{j}+c_{i} c_{j}}{l_{i}} & \frac{b_{i} b_{k}+c_{i} c_{k}}{l_{i}} \\ \frac{b_{j} b_{i}+c_{j} c_{i}}{l_{j}} & \frac{b_{j} b_{j}+c_{j} c_{j}}{l_{j}} & \frac{b_{j} b_{k}+c_{j} c_{k}}{l_{j}} \\ \frac{b_{k} b_{i}+c_{k} c_{i}}{l_{k}} & \frac{b_{k} b_{j}+c_{k} c_{j}}{l_{k}} & \frac{b_{k} b_{k}+c_{k} c_{k}}{l_{k}}\end{array}\right]$, with $b_{i}=x_{2 j}-x_{2 k}, c_{i}=x_{1 k}-x_{1 j}, A_{N}$ element area, $l_{i} i$-th edge length and $i, j, k$ node numbers (1, 2 and 3$)$; here it is worth noting that $\mathbf{B}_{N}$ is a $3 \times 3$ matrix that depends only on the finite element geometry;

- $\quad \dot{\boldsymbol{\omega}}_{N}=\left[\begin{array}{lll}\dot{\omega}_{i}^{N} & \dot{\omega}_{j}^{N} & \dot{\omega}_{k}^{N}\end{array}\right]^{T}$ are the side normal rotation rates, linked with $\dot{\omega}_{N-M}^{n n}$ by means of the linear equation $\dot{\omega}_{N-M}^{n n}=\dot{\omega}_{i}^{N}-\dot{\omega}_{i}^{M}$;

- $\quad \Gamma_{I}$ is the interface length.

The power dissipated at each interface between adjacent triangles ( $\pi^{I}$ ) may be evaluated following a general approach recently presented in the technical literature for the limit analysis of plane-strain problems (see Krabbenhoft et al. [31]) and taking into account that three different elementary interface plastic dissipations can occur, related respectively to shear $T_{n t}$ (only for the heterogeneous approach), bending moment $M_{n n}$ and torsion $M_{n t}$.

When dealing with a heterogeneous approach with joints reduced to interfaces, a linearized Lourenço and Rots failure criterion is adopted for each point belonging to the joint. The same mechanical properties assumed in the homogenized approach are assumed. In order to obtain the corresponding $T_{n t}-M_{n n}-M_{n t}$ failure surfaces for the interface, an equilibrated FE limit analysis approach is utilized, which is described in detail in [3]. The reader is referred there for further details on the elements used to discretize the interface and the linear programming approach adopted to obtain a linearization of the resultant $T_{n t}-M_{n n}-M_{n t}$ failure surface at assigned vertical pre-compression. A linearization with 80 planes of the failure surface is utilized here for the simulations, which seems reasonably accurate for the problem under consideration.

No differences occur when dealing with a heterogeneous approach with finite thickness joints, since also in this case plastic dissipation can occur only at the interface between contiguous elements. The only difference relies in the typology of interfaces which can be encountered, i.e. brick-brick, brickmortar and mortar-mortar interfaces. In particular, when a brick-mortar interface is considered, joint mechanical properties are assumed, in agreement with consolidated literature in this field [2][3]. 
Velocity boundary conditions are imposed in the model in the same way of a standard displacement based FE code and they result in additional equality constraints on nodal out-of-plane velocities.

External power dissipated may be written as $\pi_{e x t}=\left(\boldsymbol{\pi}_{0}^{T}+\lambda \boldsymbol{\pi}_{1}^{T}\right) \mathbf{U}$, where $\boldsymbol{\pi}_{0}$ is the vector of (equivalent lumped) permanent loads, $\lambda$ is the load multiplier, $\boldsymbol{\pi}_{1}^{T}$ is the vector of (lumped) variable loads and $\mathbf{U}$ is the vector of assembled nodal velocities. As the amplitude of the failure mechanism is arbitrary, a further normalization condition $\boldsymbol{\pi}_{1}^{T} \mathbf{U}=1$ is usually introduced. Hence, the external power becomes linear in $\mathbf{w}$ and $\lambda$, i.e. $P^{e x}=\boldsymbol{\pi}_{0}^{T} \mathbf{w}+\lambda$ and standard linear programming algorithms can be used to obtain collapse multipliers.

After elementary assemblage operations and considering the previously discussed constraints, the following optimization problem is obtained at a structural level:

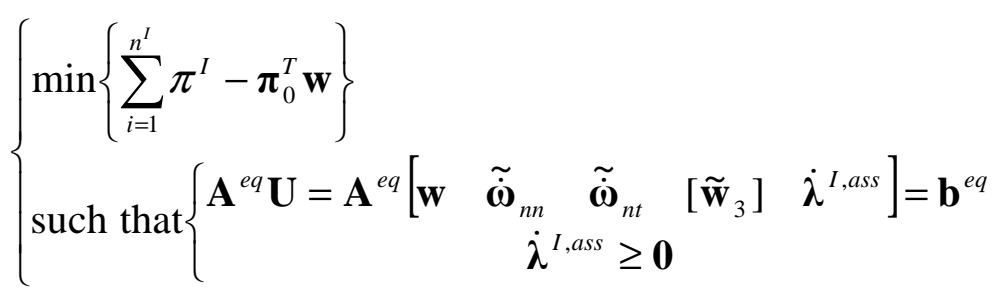

where:

- $\mathbf{U}=\left[\begin{array}{lllll}\mathbf{w} & \tilde{\dot{\boldsymbol{\omega}}}_{n n} & \tilde{\dot{\boldsymbol{\omega}}}_{n t} & {\left[\begin{array}{ll}\tilde{\mathbf{w}}_{3}\end{array}\right] \quad \dot{\lambda}^{I, \text { ass }}}\end{array}\right]$ is the vector of global unknowns, which collects the vector of assembled nodal velocities $(\mathbf{w})$, the vector of assembled bending interface rotation rates $\left(\tilde{\dot{\boldsymbol{\omega}}}_{n n}\right)$, the vector of assembled torsion interface rotation rates $\left(\tilde{\dot{\boldsymbol{\omega}}}_{n t}\right)$, the vector of assembled jumps of velocities on interfaces $\left(\left[\tilde{\mathbf{w}}_{3}\right]\right)$ and the vector of assembled interface plastic multiplier rates $\left(\dot{\lambda}^{I, \text { ass }}\right.$ ) .

- $\mathbf{A}^{e q}$ is the overall constraints matrix and collects velocity and rotation boundary conditions, equations ( 13 ) and constraints for plastic flow in velocity discontinuities.

\section{Windowed panel subject to out-of-plane pressure}

The first example analyzed here consists of a windowed wall built with solid clay brick masonry. The tests were carried out on walls with regular texture by Chong et al [33] and Southcombe et al [34] and are denoted by SB. Here, only panel SB02 is analyzed with the homogenization model proposed for the sake of conciseness (see also [32] for a detailed analysis of all the configurations experimentally tested). The panel has dimensions $5600 \times 2475 \times 102.5 \mathrm{~mm}^{3}$ (see also Figure 21-a), was built in stretcher bond between two stiff abutments with the vertical edges simply supported (allowance for in-plane displacements was provided) and the top edge free. A completely restrained 
support was provided at the base because of practical difficulties in providing a simple support. The opening sizes and dimensions used in the tests were chosen to be representative of those used in practice, see Figure 21. The panels were loaded by air-bags until failure with increasing out-ofplane uniform pressure $p$. The air pressure and the displacement $d$ of the middle point of the free edge were monitored during testing. Bricks average dimensions are assumed equal to $215 \times 65 \times 102.5 \mathrm{~mm}^{3}$, whereas joint thickness is reduced to zero within the simplified model proposed.

A frictional failure surface with limited tensile strength and cap in compression is considered (Lourenço and Rots [1] failure criterion) for mortar joints. Mechanical properties at failure adopted for mortar are given in Table II and are in agreement with data reported in Milani et al. [32] (the reader is referred there for a comprehensive discussion of the numerical values adopted, also in light of the experimental flexural uniaxial strengths reported by Chong et al [33]).

In the numerical analyses, two different patterns are considered, corresponding to blocks random dispositions depicted in Figure 2. A large set of Monte Carlo simulations is finally repeated for each pattern investigated, comparing results (collapse loads distributions and failure mechanisms) with those provided by alternative expensive heterogeneous approaches.

While the model proposed is capable of predicting collapse loads quite accurately, it does not give any information on the displacements near collapse. This drawback is typical of limit analysis, where constituent materials are assumed rigid-perfectly plastic. An elastic-plastic model based on sequential quadratic programming is under investigation for a prediction of displacements near collapse.

\subsection{No horizontal alignment (Case I disposition): homogenized analyses}

A set of Monte Carlo simulations is performed on the structural model at hand, discussing the effect of changing the coefficient of variation (COV) of the height and the length of the blocks, assuming the wall arranged through the first disposition of blocks (Case I) discussed in the previous sections. Six different values of COV for $\mathrm{H}$ and $\mathrm{L}$ are analyzed, respectively equal to 2.5-5-10-15-20-25\%. At fixed values of $\mathrm{H}$ and L COVs, a set of 2,000 Monte Carlo simulations is performed, meaning that a total of 72,000 structural analyses are repeated for the problem under consideration. In Figure 22 , results obtained from the numerical simulations are represented in terms of empirical cumulative distribution functions (ECDFs), at fixed length and height COVs. Mean values of the distributions estimated thorough the Monte Carlo simulations are also represented, along with the 
deterministic values obtained assuming a stack bond and a running bond disposition for the blocks, using respectively black, red and purple thick curves.

The following key aspects are worth noting from the distributions, confirming that the procedure proposed is able to reproduce accurately -but at a fraction of the computational time needed by standard heterogeneous approaches- the behavior of irregular assemblages of blocks near failure:

1. When a small COV for both the height and the length of the blocks (upper left diagram of Figure 22) is assumed (e.g. 2.5\%), the behavior of the wall is closer to the stack bond situation. As can be seen from Figure 2-a, due to the adopted definition of geometry, Case I arrangement reduces to a stack bond disposition when $\mathrm{H}$ and L COVs tend to zero.

2. The mean failure load and standard deviation increase with the height $\mathrm{H}$ and length L COVs. Both parameters play an important role, with a slightly more relevant role on the length: for a L COV of $5 \%$ the mean failure pressure are 2.28 and $2.53 \mathrm{kN} / \mathrm{m}^{2}$, for a $\mathrm{H} \mathrm{COV}$ of $5 \%$ and $25 \%$ respectively; for a $\mathrm{L} \mathrm{COV}$ of $25 \%$ the mean failure loads are 2.49 and $2.68 \mathrm{kN} / \mathrm{m}^{2}$, for a $\mathrm{H} \mathrm{COV}$ of $5 \%$ and $25 \%$ respectively.

3. The limiting upper bound case of the running bond texture cannot be achieved with the increasing COVs, because the extra resistance due to the full staggering of the blocks is never sampled with the database collected previously.

\subsection{Case II disposition: quasi periodic arrangement}

A second set of Monte Carlo simulations $(2,000)$ is performed assuming a quasi periodic disposition of the blocks, i.e. imposing that bricks height is constantly equal to the mean value and considering the second disposition of the blocks shown in Figure 2 (Case II, but with constant blocks height). In order to validate the results obtained with the model proposed an expensive set of heterogeneous Monte Carlo simulations is also performed, in which mortar joints are reduced to interfaces and blocks are supposed infinitely resistant.

The COV for blocks length is assumed equal to $15 \%$ whereas blocks height is assumed constantly equal to $65 \mathrm{~mm}$. Wall thickness is assumed equal to $102.5 \mathrm{~mm}$.

A typical heterogeneous discretization by means of triangular elements with interfaces discontinuities for the wall under consideration is depicted in Figure 21-b, whereas the mesh used when dealing with the homogenization approach is reported in Figure 21-c.

The aim of the example is to compare failure load distributions -namely mean value and standard deviation- and failure mechanisms provided by the homogenized and the heterogeneous model. Typically, the panel under consideration fails for the formation of a vertical yield line in correspondence of the symmetry axis of the wall, two inclined cylindrical hinges departing from the 
corners located at the base and finishing at the corners of the window and a horizontal hinge at the base. Power dissipated along both vertical and inclined yield lines is strongly dependent on blocks staggering, because it is directly connected to ultimate vertical bending $\mathrm{M}_{11}$ and torsion $\mathrm{M}_{12}$. For this reason, it is very straightforward to conclude that failure load decreases sensibly in all those situations where the variability of bricks lengths results in an insufficient staggering between two contiguous blocks rows.

From a numerical point of view, it is worth underlining here that, as a rule, a single simulation on the heterogeneous model required around 12 minutes to be performed on a PC equipped with an Intel Core 2 Duo CPU @ 2 GHz and 4 GByte RAM. The computational effort required in this case is obviously a consequence of the number of blocks present in the panel. On the contrary, when dealing with the homogenization approach less than 30 seconds were needed for a single limit analysis simulation. This justifies the relatively limited number of simulations performed $(2,000)$ to evaluate the output distributions, which in any case required around 15 days of processing time only for the heterogeneous model. Considering also that three hours were required to collect the database of failure surfaces (see previous sections) for the homogenized approach, less than one day was needed to complete the homogenized simulations, meaning that the simple two steps limit analysis procedure here proposed competes very favorably with direct models based on a distinct discretization of blocks and joints.

In Figure 23, a comparison between deformed shapes at collapse obtained with a heterogeneous mesh and the homogenized approach is represented (failure mechanisms corresponding to the average collapse load value). As it is possible to notice, the failure mechanisms are almost symmetric, because the high number of blocks used to build the specimens has the effect of equally distributing in the space asymmetries due to the random disposition of vertical joints. In any case, the failure mechanism provided by the heterogeneous model is almost perfectly reproduced by the homogenized approach. In Figure 24, the plastic multiplier of the interfaces between contiguous elements (which, in the framework of limit analysis, represents the crack pattern) is depicted using thick lines, both for the homogenized and the heterogeneous (joints reduced to interfaces) model. Again, the good agreement between models is worth noting.

Finally, in Figure 25, the failure load distribution provided by the heterogeneous and the homogenized approach are compared, along with the corresponding confidence bounds. The vertical segment reported in the figure represents the deterministic prediction obtained "at hand" adopting the orthotropic homogeneous model proposed by Sinha [35].

Furthermore, in order to throw light on the modeling of mortar joints as dimensionless interfaces rather than as a nonlinear and inelastic medium of finite size, a further set of MC simulations using 
finite thickness joints is performed. Two elements along the joint thickness are used to reproduce a failure mechanism zigzagging between blocks, as illustrated schematically in Figure 20. The corresponding ECDF is represented in Figure 25 with gray color. Comparing such result with the zero thickness distribution, it can be argued that the reduction of joints to interfaces makes sense and the error introduced through this simplification is negligible, at least for thin mortar, as is the case here treated. From the heterogeneous analysis, it can be also argued that the reduction of joints to interfaces, at a cell level and within the homogenization model proposed, seems a reasonable assumption. Finally, from an overall analysis of all ECDFs (homogeneous, heterogeneous with interfaces and heterogeneous with finite thickness joints), it can be concluded that all curves are in satisfactory agreement, with rather similar average values (around $2.5 \%$ difference).

\section{Masonry rectangular plate out-of-plane loaded}

A rectangular masonry panel of dimensions 300x200x15 $\mathrm{cm}^{3}$ (length $\mathrm{x}$ height $\mathrm{x}$ thickness) and simply supported on the four edges, see Figure 26, is considered as second example. The wall is supposed assembled with blocks of average dimensions 30x20x $15 \mathrm{~cm}^{3}$ (length $\mathrm{x}$ height $\mathrm{x}$ thickness) disposed at random following first and second disposition of Figure 2 and subsequently loaded outof-plane by means of distributed pressure.

For the problem under consideration, much less blocks are needed to build the panel: it is therefore expected that, due to the random disposition of blocks, a more marked asymmetry in the failure mechanism is obtained. As a rule, a disordered disposition of blocks with variable dimensions has visible effects on failure mechanisms only when relatively few blocks are needed to build the wall, whereas (as it occurred in the previous example) panels constituted by a large number of bricks tend to exhibit almost the same failure mechanism, even in presence of non symmetric textures (although several differences occur in the value of the failure load, which -in the framework of the yield line theory, see also Milani et al. [22] and Sinha [33]- strongly depends on bricks staggering).

In both models, for mortar joints a Lourenço and Rots [1] failure criterion is used, with mechanical properties summarized in Table III. The limited number of optimization variables involved in the heterogeneous approach allows to perform large scale Monte Carlo simulations to compare with those collected using the homogenization approach proposed.

\subsection{No horizontal alignment (Case I disposition): homogenized analyses}

As for the windowed panel, a preliminary set of Monte Carlo simulations is performed on the shear wall, discussing the effect of changing the COV of height and length blocks and assuming a 
disposition of blocks without horizontal alignment (Case I arrangement). Six different values of $\mathrm{COV}$ for $\mathrm{H}$ and $\mathrm{L}$ are investigated, respectively equal to $2.5-5-10-15-20-25 \%$. At fixed values of $\mathrm{H}$ and L COVs, 2,000 structural analyses are repeated in order to obtain empirical cumulative distribution functions (ECDFs) of the failure load from the homogenized model. In Figure 27, numerical simulations results are depicted, representing ECDFs of the failure total shear at the base corresponding to fixed length and height COVs. Estimated distributions mean values, deterministic failure loads assuming a running bond and a stack bond disposition are also represented, using respectively black, red and purple thick curves.

Similarly to the previous example, when a COV near to zero for both the height and the length of the blocks (upper left diagram of Figure 27) is considered, the wall approximates a stack bond disposition. Intuitively, failure load standard deviation tends to increase increasing block dimensions COVs. Analogously to the previous case, the upper bound is not theoretically represented by a running bond texture, because an extra resistance (both in torsion and vertical bedding) may be present in Case I, due to full staggering of the blocks. Moreover, the stack bond lower bound is well approximated by the low COVs, which is again due to the adopted definition of geometry, and not necessarily represents the physics of irregular masonry bonds.

\subsection{Case II disposition: quasi periodic arrangement}

A second sub-set of Monte Carlo simulations is performed for the case at hand, assuming a quasiperiodic blocks disposition (Case II of Figure 2), with a COV for bricks length equal to $15 \%$. Meshes used for the heterogeneous and the homogenized limit analyses are depicted in Figure 26. The aim of the example is to compare failure loads and failure mechanisms provided by the homogenized and the heterogeneous model in a case where the collapse is due to the formation of inclined yield lines, i.e. where a non trivial combination of ultimate bending and torsion contribute to the overall resistance of the wall.

Due to the relatively small number of elements required for a heterogeneous discretization, only 1 minute and less than 10 seconds were required for a single heterogeneous and homogenized Monte Carlo simulation respectively. Hence, a total of 10,000 simulations to evaluate the output collapse load distribution have been performed, requiring around 7 days of processing time for the heterogeneous approach. Also in this case, the homogenization model competes favorably with micro-modeling, requiring only 2 days, comprising 10,000 simulations and the preliminary evaluation of the stochastic failure surface.

In Figure 28, a comparison between deformed shapes at collapse obtained with a heterogeneous mesh and the homogenized one is reported (failure mechanisms correspond to the average collapse 
load values). As it is possible to notice, there is a satisfactory agreement between deformed shapes provided by the heterogeneous and the homogenized, meaning that the procedure proposed may be used for a reliable evaluation of failure mechanisms and collapse loads. In Figure 29, the plastic multiplier of interfaces between contiguous elements is represented with thick lines both for the homogenized and the heterogeneous model. Two aspects are worth noting here, namely the quite evident asymmetry of the zones of dissipation and the good results provided by the homogenized approach. Finally, in Figure 30, failure loads distributions provided by the heterogeneous and the homogenized model are represented, with the corresponding confidence bounds. Again, the distribution provided by the homogenized approach is very similar to the heterogeneous one, with comparable average values and standard deviations.

\section{Romanesque masonry church façade out-of-plane loaded}

As final application of the model proposed on a real scale structure, a full façade of a small church located in Portugal (see Figure 31) and built with large stones in irregular texture is considered. Its resistance for out-of-plane partial collapses, which are very frequent under seismic excitation, is investigated through different FE and "at hand" kinematic limit analysis models. The example here presented is part of a research project still running at the University of Minho (see [36][37]) and dealing with the seismic safety assessment of a large set of historical masonry churches in Portugal. The church here considered is a typical example of Romanesque architecture in Portugal: for this reason, it appears rather interesting to analyze its resistance when subjected to out-of-plane loads. Approximately, the façade has a width of 8.50 meters, a maximum height of 7.80 meters and an average thickness of $600 \mathrm{~mm}$. The façade is built with stones disposed irregularly with variable offset between contiguous rows. Three different numerical models are here compared. The first is a heterogeneous FE limit analysis model with blocks disposed in irregular texture, the second is the same heterogeneous model but with a deterministic disposition of the blocks in running bond, whereas the third is the homogenized stochastic model proposed in this paper. Here it is worth noting that, due to the relevant thickness of the façade, a numerical model based on the utilization of Kirchhoff-Love plate elements would give inaccurate results. Therefore, the same FE model used for the previous examples is utilized here, but with finite out-of-plane shear strength. FE triangular discretizations adopted in the paper for the simulations are sketched in Figure 32, where different gray scales indicate different blocks. In the simulations, joints are reduced to interfaces with a Lourenço and Rots [1] failure criterion (cohesion c $=0.05 \mathrm{MPa}, \Phi=30^{\circ} f_{t}=c / \tan \Phi, f_{c}=30 f_{t}, \Phi_{1}$ $=60^{\circ}$ ), whereas blocks are assumed infinitely resistant. Here, it is worth noting that a very low tensile strength is assumed for joints, which is typical for this typology of structures (see [36][37]). 
A good interlocking between the façade and perpendicular walls is also assumed, in order to investigate two-dimensional failure mechanisms involving out-of-plane failure only with inclined yield lines.

In order to have a large set of data to compare and with the aim of testing the reliability of the FE limit analysis approach proposed, collapse loads obtained numerically are compared with that provided by "at hand" calculations. In this latter case, it has been shown [38][39] that one of the possible failures which can be observed after earthquake surveys (and corresponding to the minimum collapse multiplier) is a second type failure mechanism by Rondelet. It involves the upper part of the façade and represents schematically a rocking motion of the gable. Typically, two symmetric inclined yield lines departing from the East and West upper edges crossing at the top of the entrance and a vertical yield line on the symmetry axis departing from the upper edge define the failure mechanism. In agreement with consolidated literature in this field (see [38][39][40]) and with some codes of practice (e.g. [41]), the wall is assumed isotropic and without tensile resistance. In this framework, closed form expressions available in the technical literature [38][39] may be used for a fast an conservative evaluation of the collapse multiplier.

In Figure 33, a comparison between collapse loads ( $\mathrm{a}_{\mathrm{g}}$ indicates the horizontal out-of-plane acceleration at collapse, whereas $\mathrm{g}$ is the gravity acceleration) provided by the different models analyzed is shown. When dealing with the heterogeneous irregular texture and the homogenized approach, full MC simulations (10000 samples) are performed and in the figure the corresponding ECDFs are represented. At hand prediction and the numerical deterministic running bond failure load are also represented in order to compare the different results obtained through all models.

In Figure 34, a comparison among deformed shapes at collapse from FE limit analysis simulations (corresponding to mean values simulations where MC is used) is represented. Finally, in Figure 35, crack patterns are compared.

From an overall analysis of the results obtained, several key aspects are underlined next. As expected, the safety factor of the façade for out-of-plane partial collapses is largely insufficient, meaning that rehabilitation interventions might be needed for similar churches located in high seismic areas.

The ECDFs of the collapse load corresponding to the homogenized and the heterogeneous FE models are very close, meaning that the homogenized model proposed may be used for large scale structural computations at a fraction of the time needed for heterogeneous analyses.

Both homogenized and heterogeneous mean values of the collapse load overestimate the value found manually assuming two inclined yield lines and a vertical cylindrical hinge. This is not 
surprising, since, in this latter case, masonry material is considered isotropic with no tensile resistance. Consequently, safe predictions are obtained.

The collapse load obtained manually assuming a simple rocking of the gable (first mode partial collapse), see Figure 33, is strongly overestimated. Similar considerations can be repeated for the value corresponding to the FE numerical model with deterministic running bond disposition of the blocks. In this case, failure mechanism seems to involve a diffused zone under the entrance lintel. This result is again not surprising. As already pointed out, in fact, upper bounds for the masonry strength domain are obtained with a deterministic running bond pattern.

From a comparative analysis of failure mechanisms and crack patterns (given by the maps of plastic dissipation on interfaces for out-of-plane actions) of random models (both homogenized and heterogeneous), it is particularly evident the formation of two inclined yield lines and a vertical cylindrical hinge near the symmetry axis. The overall failure mechanism results in good agreement with the manual approach suggested by the Italian code [41]. Furthermore, the inclination angle of the yield lines suggested by the crack pattern seems rather in agreement with observed collapses occurred after seismic events [40].

The asymmetry in the failure mechanisms for both the homogenized and the heterogeneous model obviously depend on the random disposition of the blocks, which is considered in the homogenized model in the evaluation of masonry strength domain.

\section{Conclusions}

A homogenized rigid-plastic plate model for a fast and reliable analysis of masonry structures loaded out-of-plane and constituted by blocks disposed in irregular texture has been presented. A two-step approach has been developed, relying in a preliminary homogenization of the random assemblage of bricks, followed by structural Monte Carlo homogenized FE analyses.

At a cell level, a generic block (supposed rigid infinitely resistant) interconnected with its neighbors by means of zero thickness mortar joints has been considered. A sub-class of possible elementary deformation modes (mimicking horizontal and vertical stretching, a pure shear deformation, pure bending and torsion along material axes) acting in the unit cell has been a priori chosen in order to describe joints cracking under normal and tangential actions. Then, power dissipated in the discrete model has been equated to that dissipated in a continuum macroscopic $2 \mathrm{D}$ equivalent plate (identification).

Two different dispositions of blocks in the REV have been studied (both obtained through blocks with variable length and height), the first relying in a random assemblage without preferential horizontal or vertical disposition lines, the second following a preferential horizontal disposition, 
thus preserving the continuity of the horizontal joint. At a fixed geometry for the REV, out-of-plane failure surfaces have been numerically evaluated solving a number of simple linear programming problems on the elementary cell. Several numerical simulations have been repeated at large scale (in the framework of a Monte Carlo approach) considering both dispositions and sampling a number of different REVs at random. Fixing the elementary cell geometry, resultant $\mathrm{M}_{11}-\mathrm{M}_{22}$ and $\mathrm{M}_{11}-\mathrm{M}_{12}$ failure surface sections have been collected and compared with those obtained sampling a different REV. This procedure has been repeated several times, thus making possible to evaluate failure surfaces stochastic distributions. By means of the database collected, comparisons at a structural level were also possible.

The original contribution of the paper relies in the possibility to provide predictions of the ultimate behaviour of entire irregular masonry structures with a very limited computations effort. The main advantage of the simple approach proposed - with respect to traditional homogenization based on FE discretizations of the unit cell - is the capability to repeat quickly a number of simulations with several different textures (generated randomly) of the REV and hence the possibility to obtain estimates of masonry strength distributions (mean values along different load directions, upper and lower bounds, standard deviation, etc.) to implement at a structural level, at a fraction of the time needed by standard procedures.

\section{References}

[1] Lourenço PB, Rots J (1997). A multi-surface interface model for the analysis of masonry structures. Journal of Engineering Mechanics ASCE 123 (7): 660-668.

[2] Milani G (2008). 3D upper bound limit analysis of multi-leaf masonry walls. International Journal of Mechanical Sciences 50: 817-836.

[3] Milani G, Zuccarello FA, Olivito RS, Tralli A (2007). Heterogeneous upper-bound finite element limit analysis of masonry walls out-of-plane loaded. Computational Mechanics 40 (6): 911-931.

[4] Lotfi HR, Shing PB (1994). Interface model applied to fracture of masonry structures. Journal of Structural Engineering ASCE 120(1): 63-80.

[5] Milani G, Lourenço PB (2009). Monte Carlo homogenized limit analysis model for randomly assembled blocks in-plane loaded. Under review.

[6] Pegon P, Anthoine A (1997). Numerical strategies for solving continuum damage problems with softening: application to the homogenization of masonry. Computers and Structures 64(4): 623-642. 
[7] Luciano R, Sacco E (1997). Homogenization technique and damage model for old masonry material. International Journal of Solids and Structures 34(4): 3191-208.

[8] Pietruszczak S, Ushaksaraei R (2003). Description of inelastic behaviour of structural masonry. International Journal of Solids and Structures 40: 4003-4019.

[9] Massart T, Peerlings RHJ, Geers MGD (2004). Mesoscopic modeling of failure and damageinduced anisotropy in brick masonry. European Journal of Mechanics A/Solids 23: 719-735.

[10] Mercatoris BCN, Massart TJ (2009). Assessment of periodic homogenisation-based multiscale computational schemes for quasi-brittle structural failure. International Journal for Multiscale Computational Engineering 7(2): 153-170.

[11] Cluni F, Gusella V (2004). Homogenization of non-periodic masonry structures. International Journal of Solids and Structures 41: 1911-1923.

[12] Falsone G, Lombardo M (2007). Stochastic representation of the mechanical properties of irregular masonry structures. International Journal of Solids and Structures 44: 8600-8612.

[13] Cecchi A, Sab K (2009). Discrete and continuous models for in plane loaded random elastic brickwork. European Journal of Mechanics A-Solids 28: 610-625.

[14] Gusella V, Cluni F (2006). Random field and homogenization for masonry with nonperiodic microstructure. Journal of Mechanics of Materials and Structures 1(2): 357-386.

[15] Cecchi A, Sab K (2009). A homogenized Love-Kirchhoff model for out-of-plane loaded Random 2D lattices. Application to Brickwork Panels. International Journal of Solids and Structures 46(14-15): 2907-2919.

[16] Lombardo M, Zeman J, Šejnoha M, Falsone G. Stochastic modeling of chaotic masonry via mesostructural characterization. International Journal for Multiscale Computational Engineering 7(2): 171-185.

[17] Zeman J, M. Šejnoha M (2007). From random microstructures to representative volume elements. Modelling Simulation Mater. Sci. Eng. 15(4): 325-335.

[18] Šejnoha J, Šejnoha M, Zeman J, Sýkora J, Vorel J (2008). A mesoscopic study on historic masonry. Structural Engineering \& Mechanics 30 (1): 99-117.

[19] de Buhan P, de Felice G (1997). A homogenization approach to the ultimate strength of brick masonry. Journal of the Mechanics and Physics of Solids 45 (7): 1085-1104.

[20] Ferris M, Tin-Loi F (2001). Limit analysis of frictional block assemblies as a mathematical program with complementarity constraints. Int. J. Mech. Sci. 43: 209-224. 
[21] Orduña A, Lourenço PB (2005). Three-dimensional limit analysis of rigid blocks assemblages. Part I: Torsion failure on frictional joints and limit analysis formulation. Int. J. Solids and Structures 42 (18-19): 5140-5160.

[22] Milani G, Lourenço PB, Tralli A (2006). Homogenised limit analysis of masonry walls. Part I: failure surfaces. Computers and Structures 84 (3-4): 181-195.

[23] Milani E, Milani G, Tralli A (2008). Limit analysis of masonry vaults by means of curved shell finite elements and homogenization. International Journal of Solids and Structures 45: $5258-5288$.

[24] Cecchi A, Milani G (2008). A kinematic FE limit analysis model for thick English bond masonry walls. International Journal of Solids and Structures 45(5): 1302-1331.

[25] Milani G (2009). Homogenized limit analysis of FRP-reinforced masonry walls out-of-plane loaded. Computational Mechanics 43: 617-639.

[26] Begg D, Fishwick R (1995). Numerical analysis of rigid block structures including sliding. In: Middleton J, Pande G (Eds.), Computer Methods in Structural Masonry 3: 177-183.

[27] Suquet P (1983). Analyse limite et homogeneisation. Comptes Rendus de l'Academie des Sciences - Series IIB - Mechanics 296: 1355-1358.

[28] Sab K (2003). Yield design of thin periodic plates by a 30omogenization technique and an application to masonry walls. C.R. Mechanique 331: 641-646.

[29] Makrodimopoulos A, Martin CM (2006). Lower bound limit analysis of cohesive-frictional materials using second-order cone programming. International Journal for Numerical Methods in Engineering 66 (4): 604-634.

[30] Krabbenhoft K, Lyamin AV, Sloan SW (2007). Formulation and solution of some plasticity problems as conic programs. International Journal of Solids and Structures 44: 1533-1549.

[31] Krabbenhoft K, Lyamin AV, Hjiaj M, Sloan SW (2005). A new discontinuous upper bound limit analysis formulation. International Journal for Numerical Methods in Engineering 63: 1069-1088.

[32] Milani G, Lourenço PB, Tralli A (2006). Homogenization approach for the limit analysis of out-of-plane loaded masonry walls. Journal of Structural Engineering ASCE, 132(10): 16501663. 
[33] Chong VL, Southcombe C, May IM (1994). The behaviour of laterally loaded masonry panels with openings. Proc., 3rd Int. Masonry Conf., Proc. British Masonry Society, London, 178182.

[34] Southcombe C, May IM, Chong VL (1995). The behaviour of brickwork panels with openings under lateral load. Proc., $4^{\text {th }}$ Int. Masonry Conf. Proc., British Masonry Society, Vol. 1, London, 105-110.

[35] Sinha BP (1978) A simplified ultimate load analysis of laterally loaded model orthotropic brickwork panels of low tensile strength. Struct Eng 56B(4):81-84.

[36] Lourenço PB, Roque JA (2006). Simplified indexes for the seismic vulnerability of ancient masonry buildings. Construction and Building Materials 20: 200-208.

[37] Roque JA (2002). Strengthening and structural rehabilitation of old masonry walls (in Portuguese) / Reforço e reabilitação estrutural de paredes antigas de alvenaria, MSc thesis, Universidade do Minho.

[38] Beolchini G, Milano L, Antonacci E (2006). Regione Molise (Italy). Decreto 76 03/08/2005. Protocollo di progettazione per la realizzazione degli interventi di ricostruzione post-sisma sugli edifici privati.

[39] Brandonisio G (2007). Analisi di edifici a pianta basilicale soggetti ad azioni sismiche. $\mathrm{PhD}$ Thesis, University of Naples

[40] Doglioni F, Petrini V, MorettiA (editors) (1994).Le chiese e il terremoto [Churches and earthquake]. Trieste, Italy: LINT Press.

[41] O.P.C.M. 3431/05. 09/05/2005. Ulteriori modifiche ed integrazioni all'OPCM 3274/03 [in Italian]. 


\section{Figures}

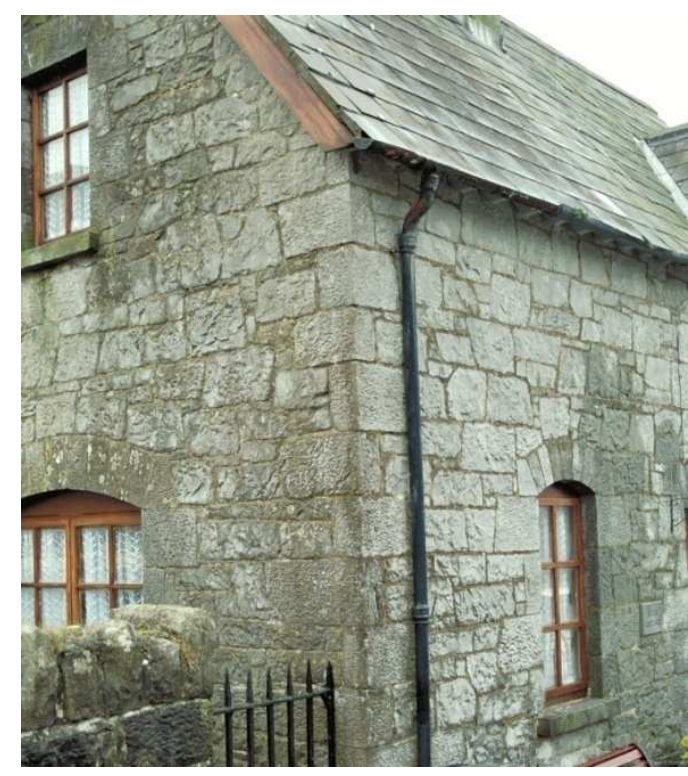

$-\mathrm{a}$

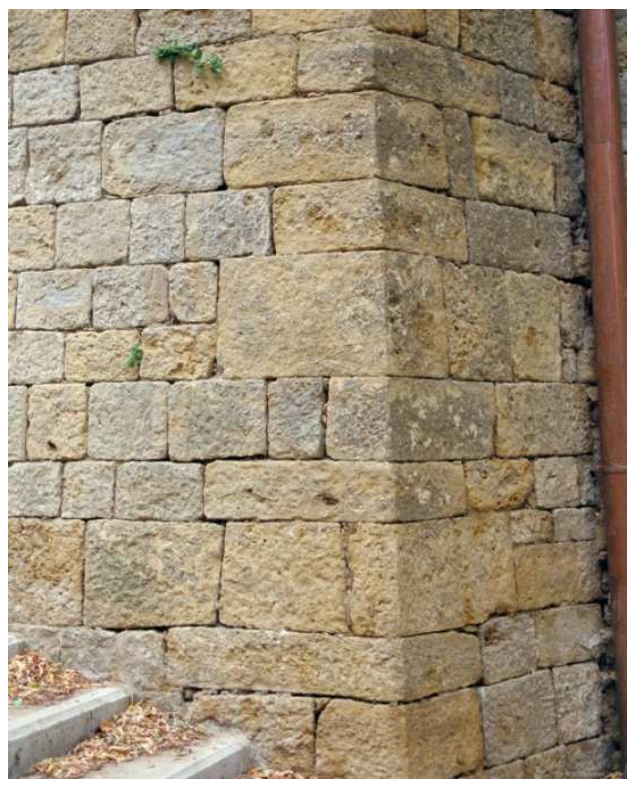

$-b$

Figure 1: Typical random patterns in existing historical buildings. -a: without horizontal alignment (Ireland). -b: with horizontal alignment (Italy).

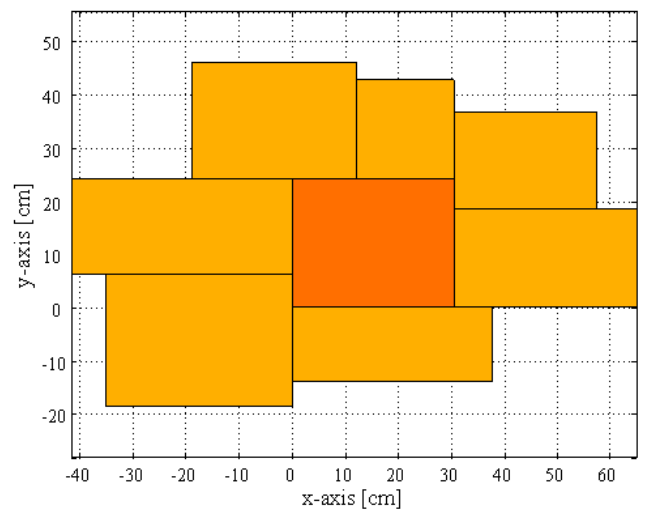

$-\mathrm{a}$

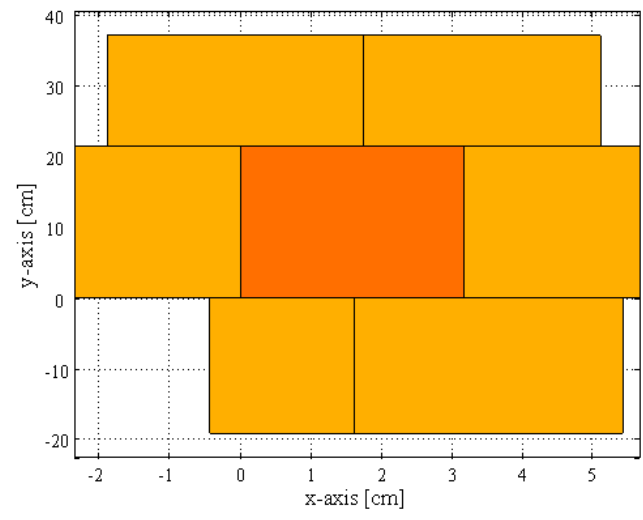

$-\mathrm{b}$

Figure 2: Randomly generated REVs. -a: without horizontal alignment (Case I). -b: with horizontal alignment (Case II). 


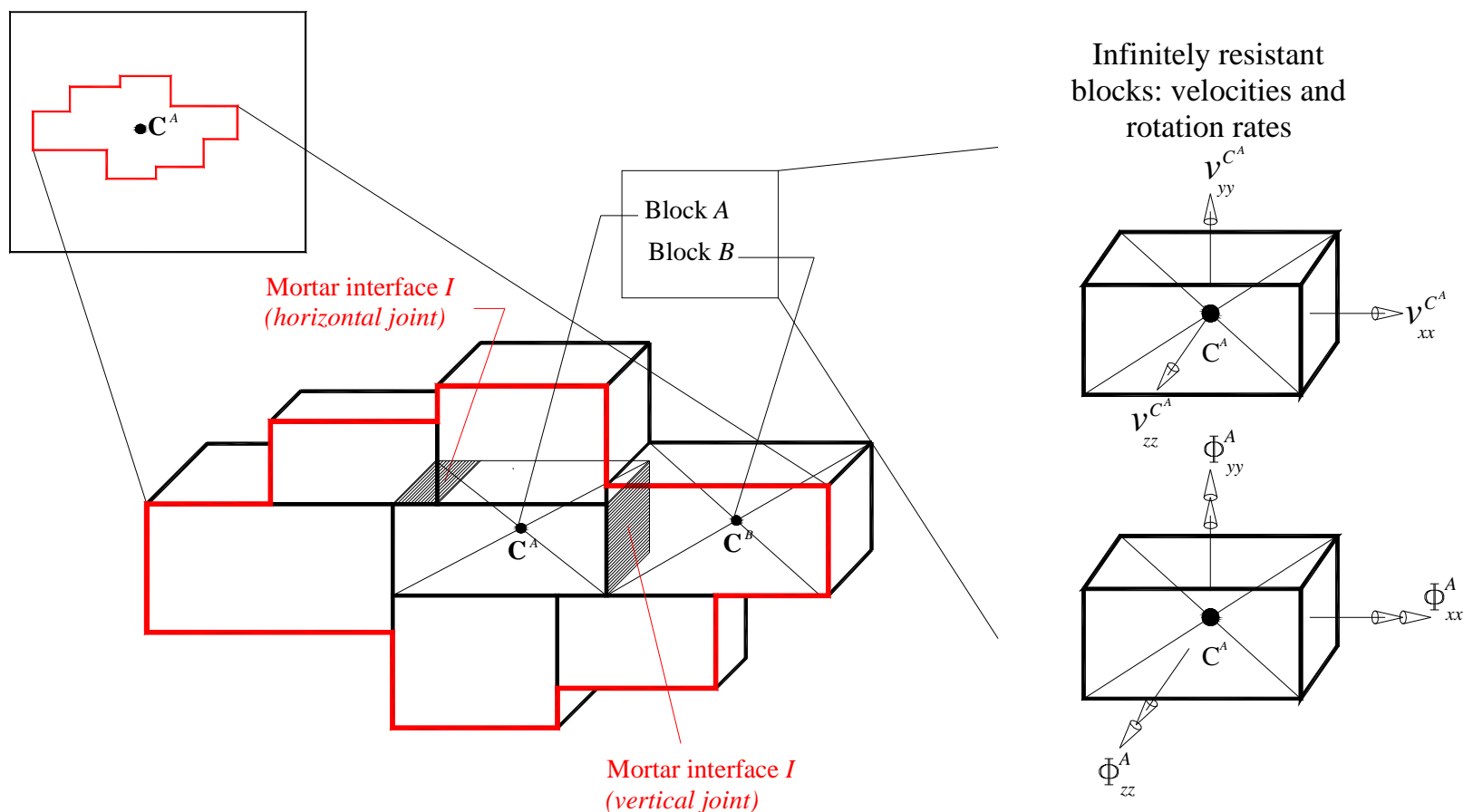

Figure 3: Masonry 3D blocks assemblage. Two adjacent blocks ( $A$, centroid $\mathbf{C}^{A}$, and $B$, centroid $\mathbf{C}^{B}$ ) are connected by means of a rigid-plastic mortar interface $I$. Blocks are rigid infinitely resistant. Three velocities unknowns and three rotation rates must be introduced in the optimization problem at a cell level for each block of the REV.

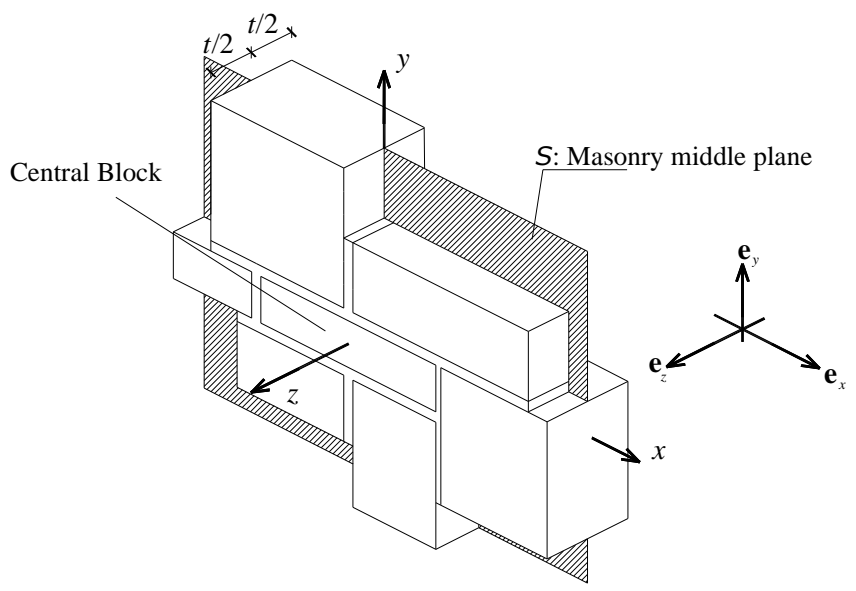

Figure 4: Reference surface chosen for masonry. 


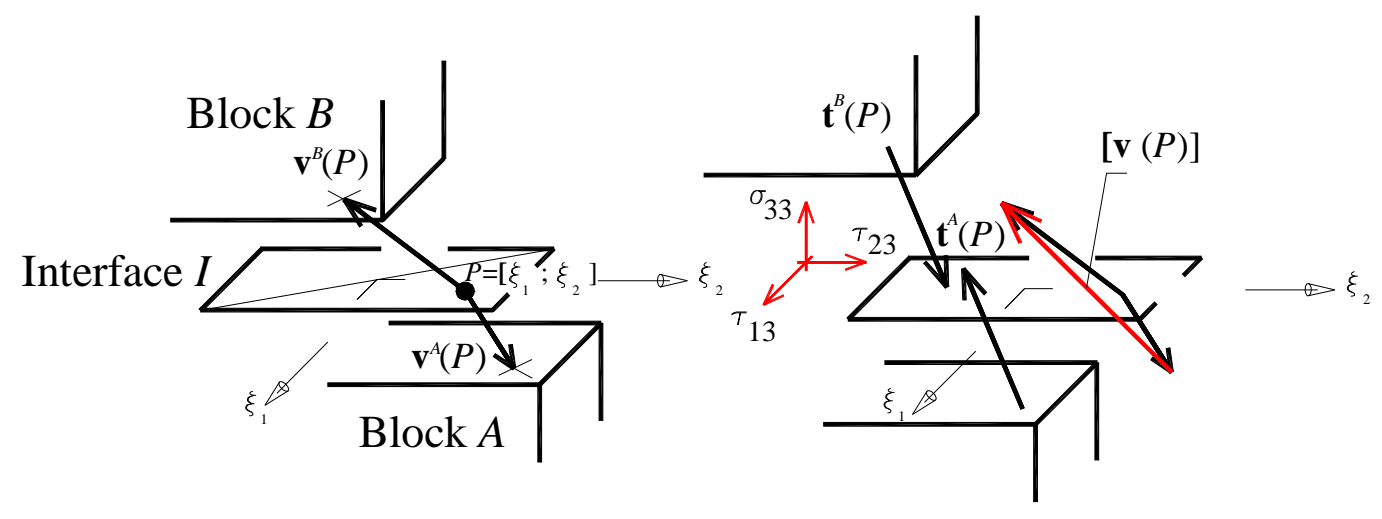

Figure 5: Jump of velocities and stress field acting on an interface $I$ between contiguous blocks $A$ and $B$. 
Power dissipated $3 \mathrm{D}$ assemblage $=$ Power dissipated $2 \mathrm{D}$ plate
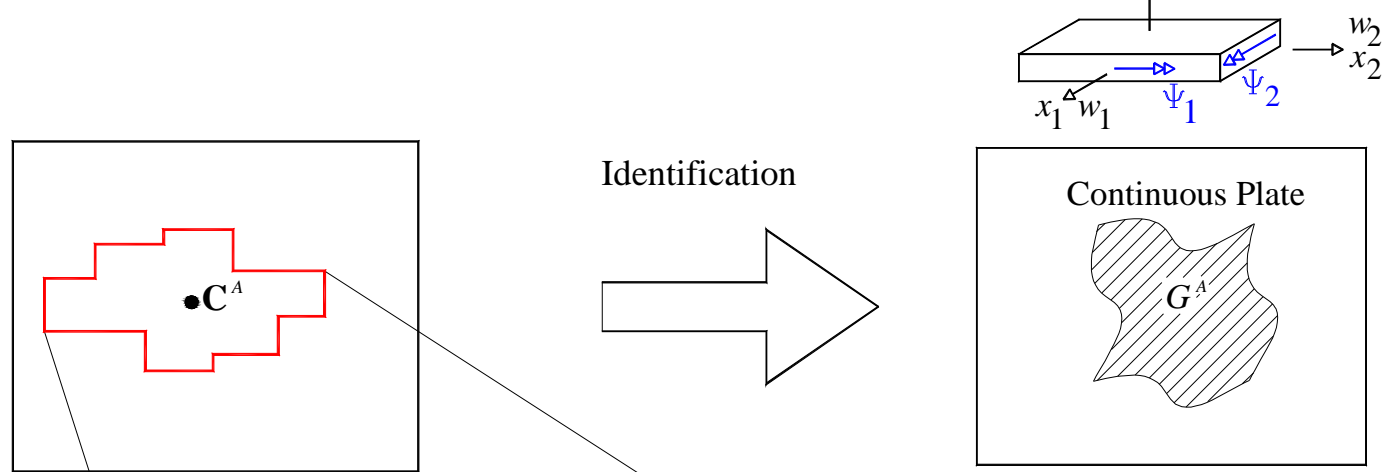

Mortar interface $I$ internal to the REV

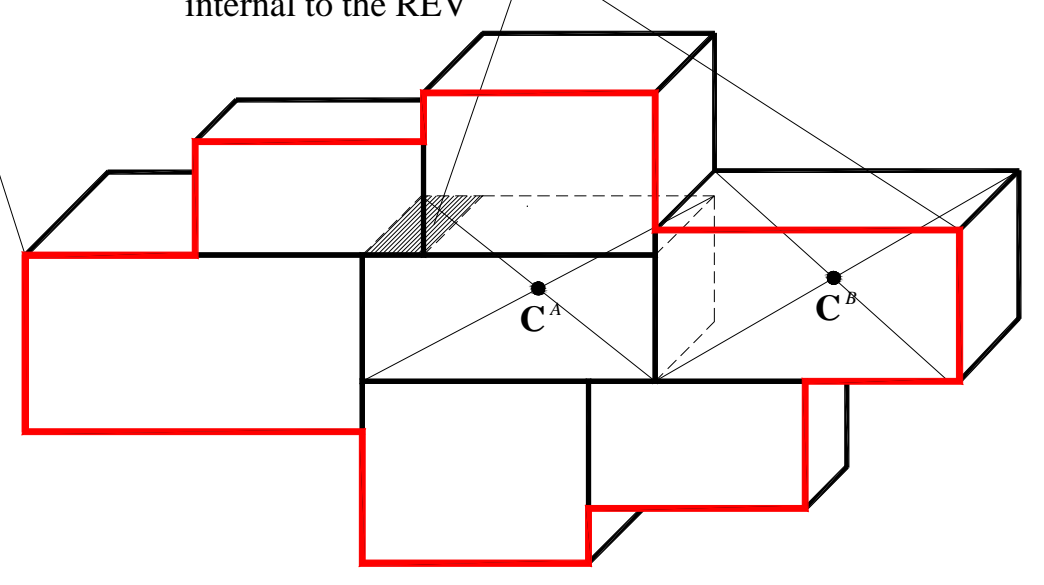

Figure 6: Representative volume element and transformation of discrete model into a continuous model. 


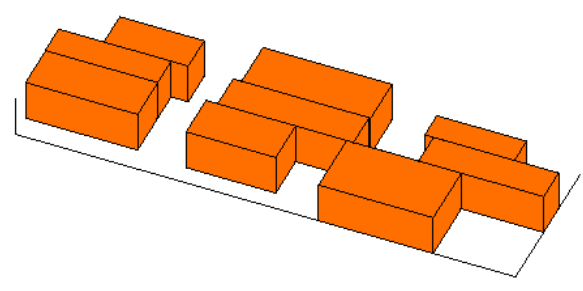

$-\mathrm{a}$

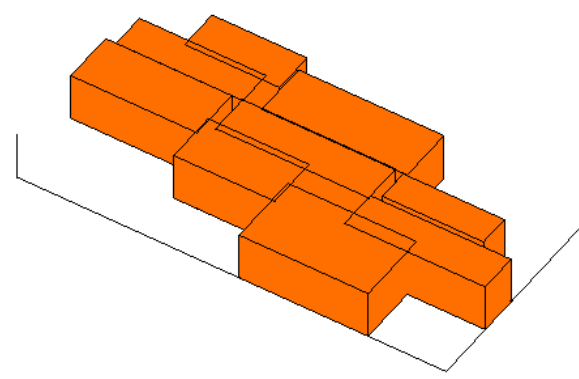

$-b$
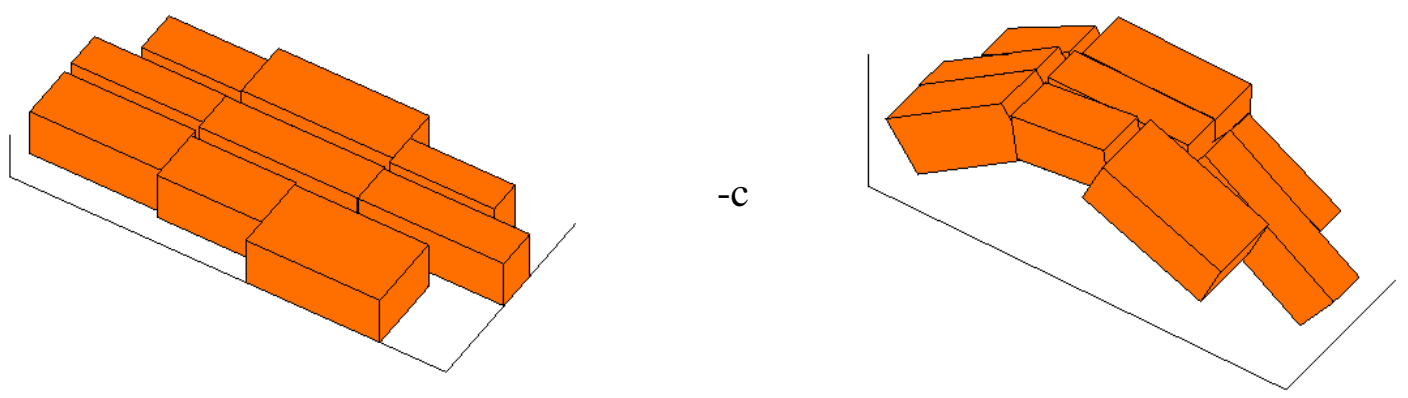

$-d$
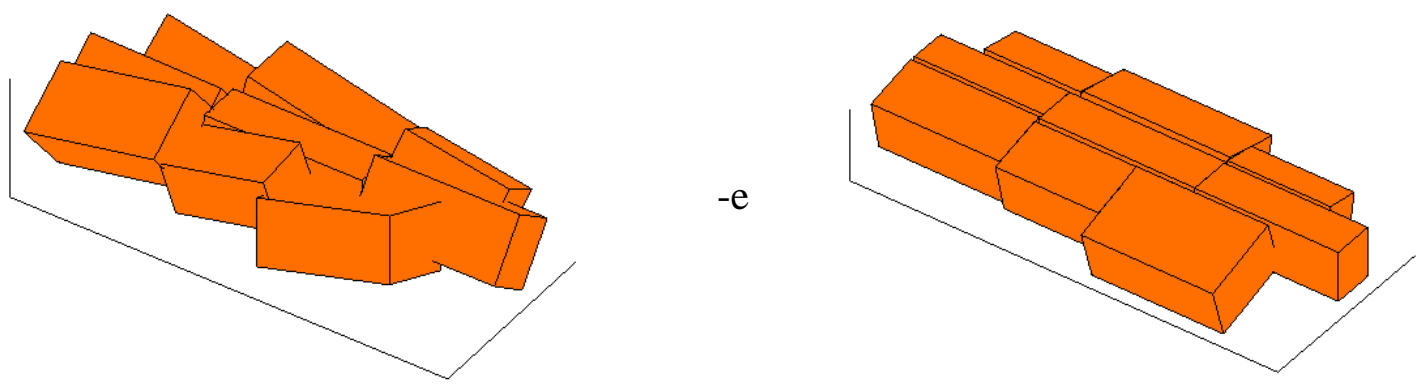

$-f$

Figure 7: Elementary homogeneous deformations applied to the representative volume element. -a: $\dot{E}_{11} \cdot-\mathrm{b}: \dot{E}_{12} \cdot-\mathrm{c}: \dot{E}_{22} \cdot-\mathrm{d}: \dot{\chi}_{11},-\mathrm{e}: \dot{\chi}_{12} \cdot-\mathrm{f}: \dot{\chi}_{22}$. 

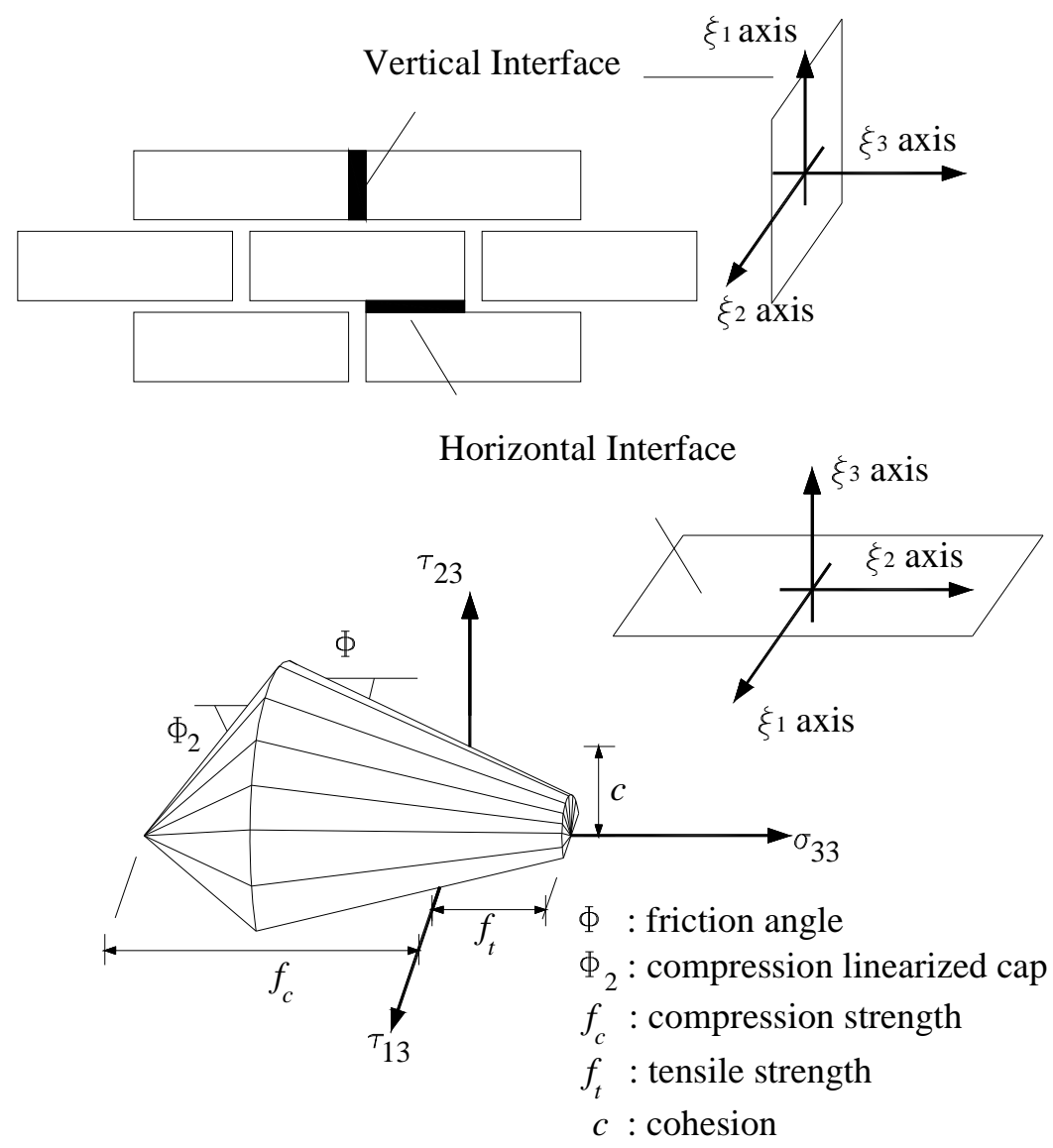

Figure 8: Piecewise linear approximation of the failure criterion adopted for joints. MohrCoulomb failure criterion with tension cut-off and linearized compression cap. 


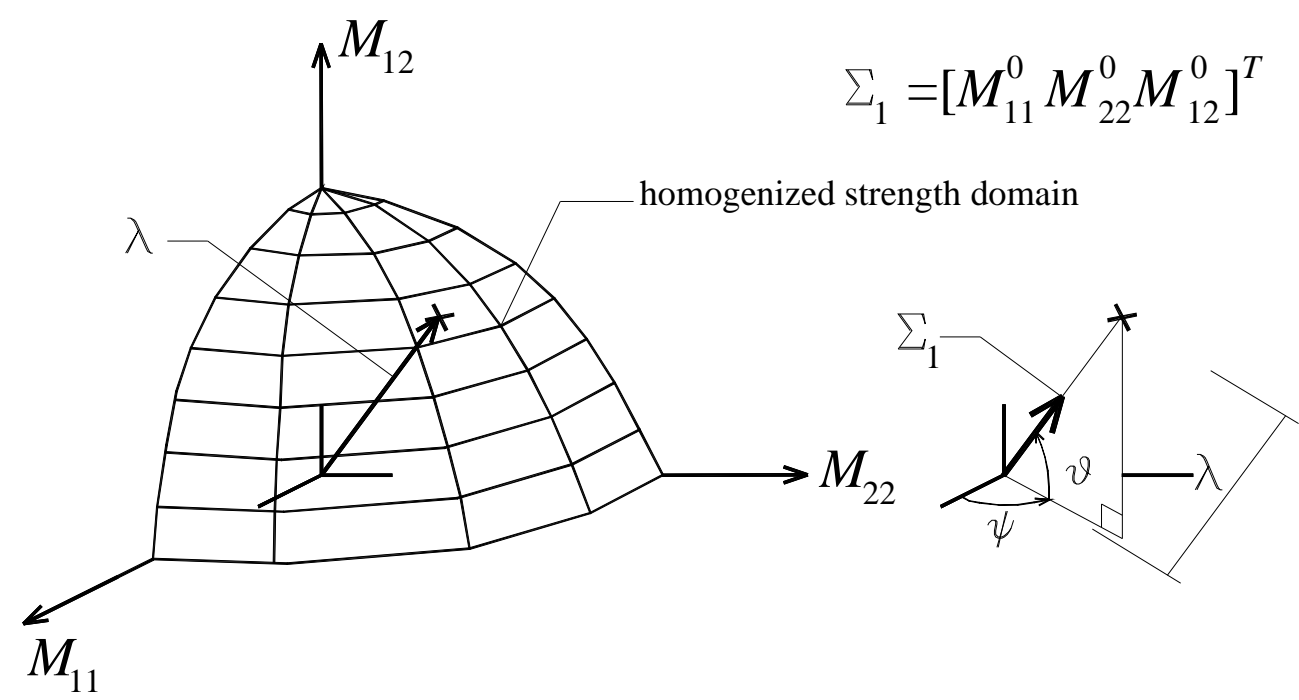

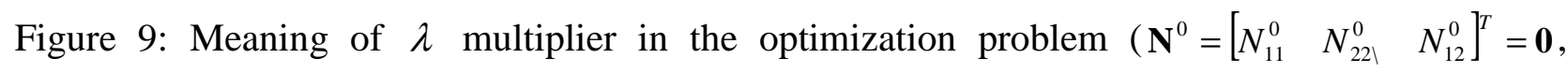
$\mathbf{T}^{0}=\left[\begin{array}{ll}T_{13}^{0} & T_{23}^{0}\end{array}\right]^{T}=\mathbf{0}$ and $\left.\mathbf{M}^{0}=\left[\begin{array}{lll}M_{11}^{0} & M_{221}^{0} & M_{12}^{0}\end{array}\right]^{T}=\mathbf{\Sigma}_{1}\right)$. 

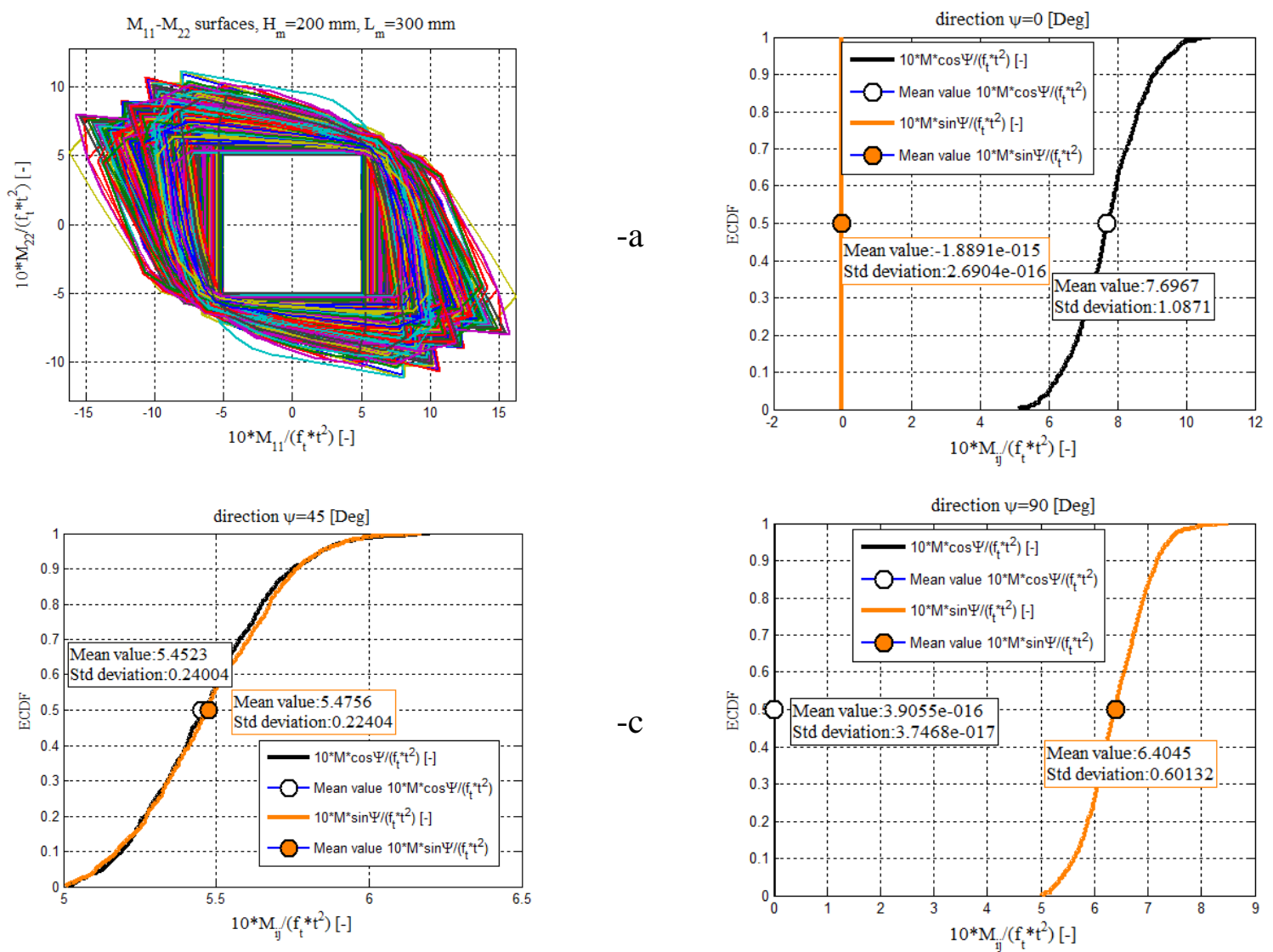

Figure 10: Case I results, $\mathrm{M}_{11}-\mathrm{M}_{22}$ sections assuming for joints a Lourenço-Rots [1] failure criterion.

Monte Carlo failure surfaces and corresponding ECDF at fixed load paths given by the $\psi$ angle. 

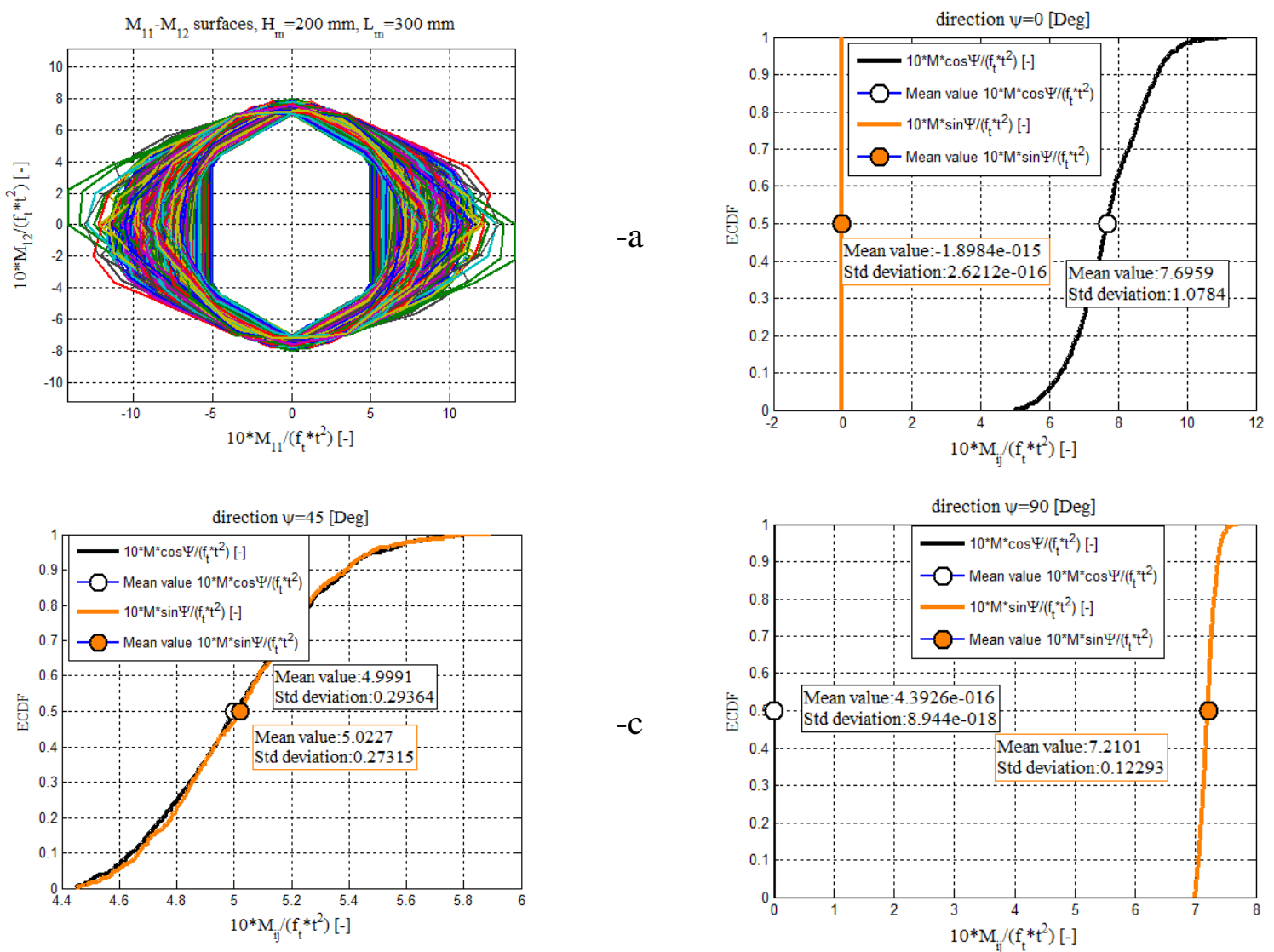

Figure 11: Case I results, $\mathrm{M}_{11}-\mathrm{M}_{12}$ sections assuming for joints a Lourenço-Rots [1] failure criterion. Monte Carlo failure surfaces and corresponding ECDF at fixed load paths given by the $\psi$ angle. 

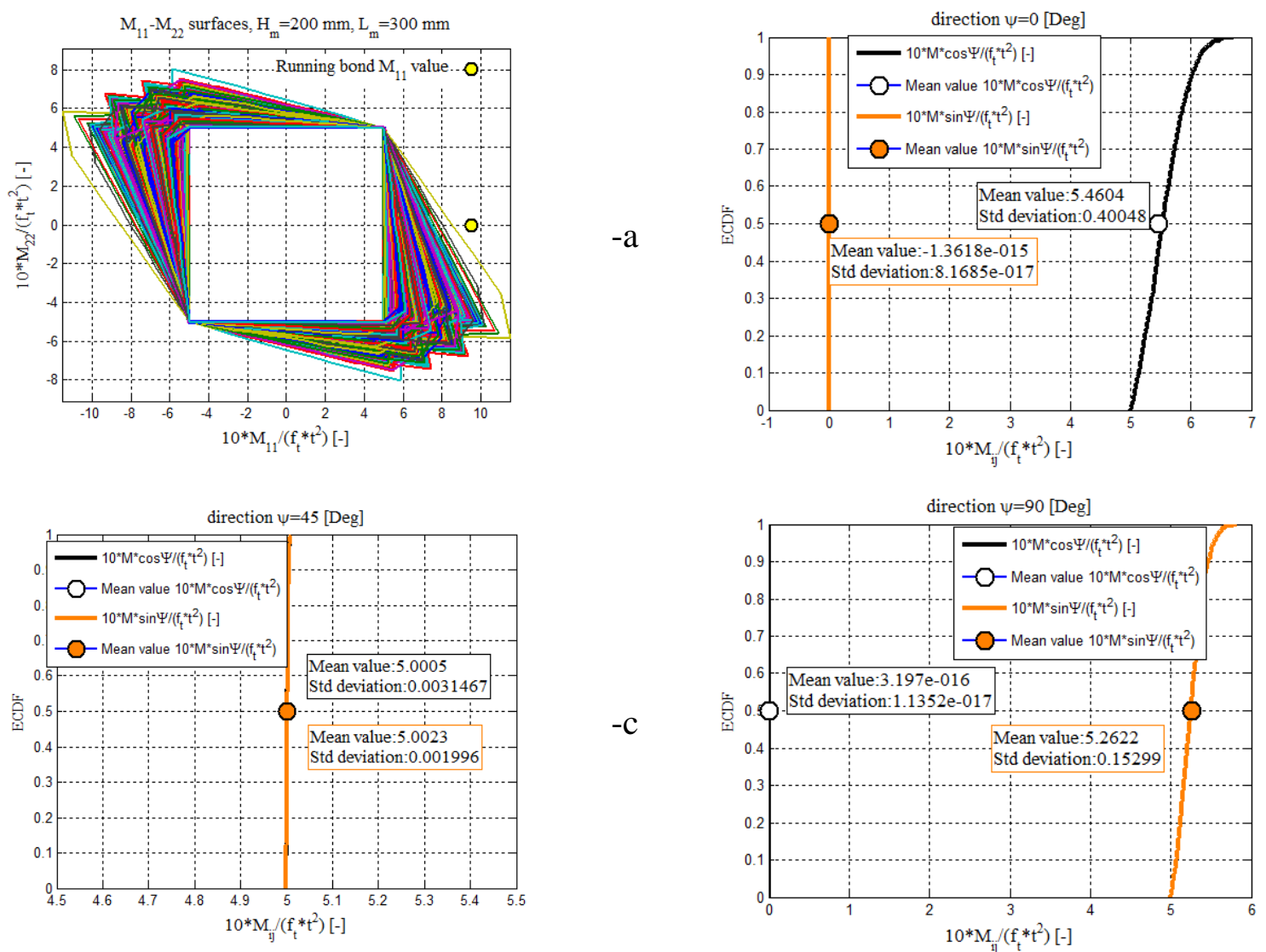

Figure 12: Case I results, $\mathrm{M}_{11}-\mathrm{M}_{22}$ sections assuming for joints a classic Mohr-Coulomb failure criterion. Monte Carlo failure surfaces and corresponding ECDF at fixed load paths given by the $\psi$ angle. 

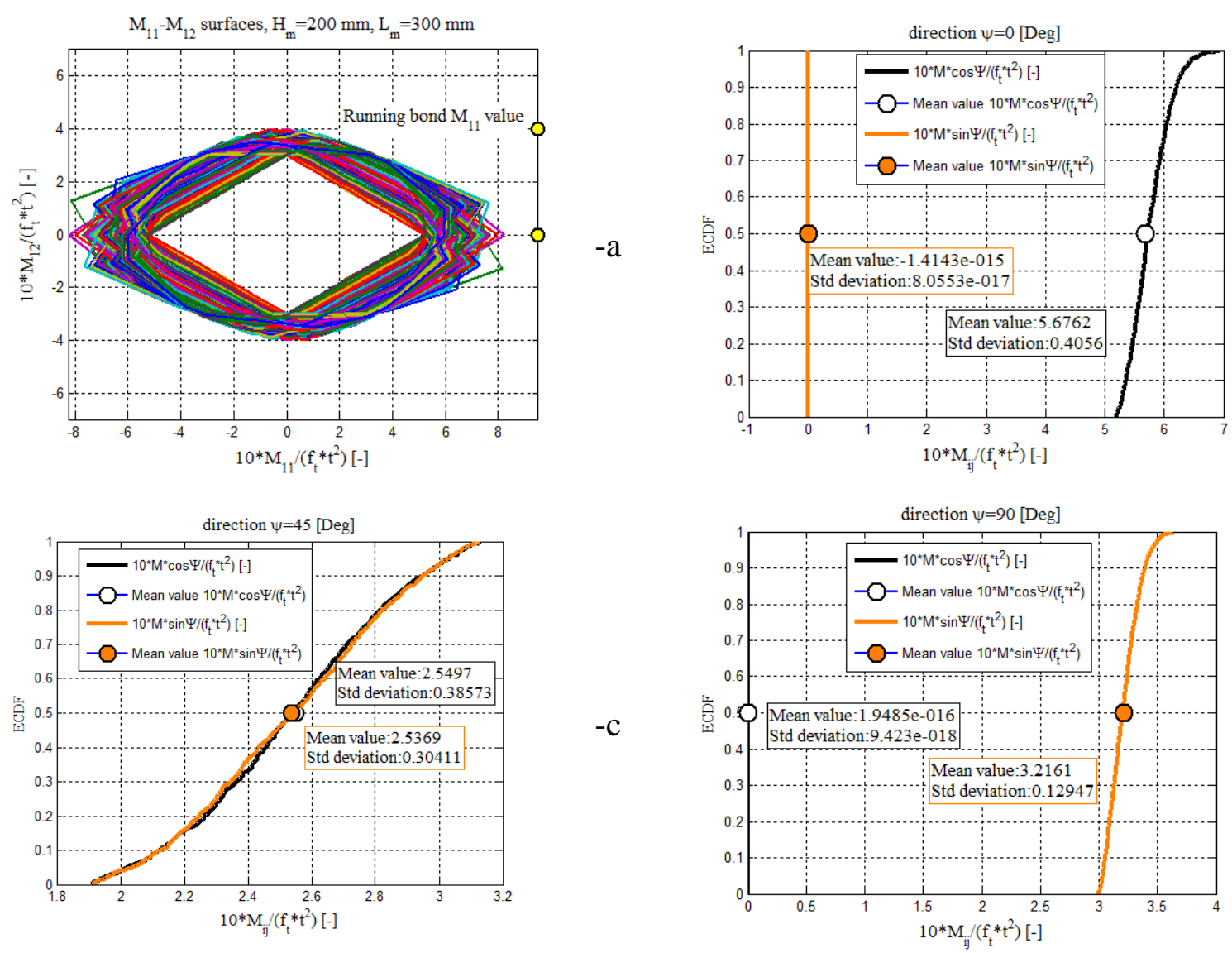

Figure 13: Case I results, $\mathrm{M}_{11}-\mathrm{M}_{12}$ sections assuming for joints a classic Mohr-Coulomb failure criterion. Monte Carlo failure surfaces and corresponding ECDF at fixed load paths given by the $\psi$ angle. 


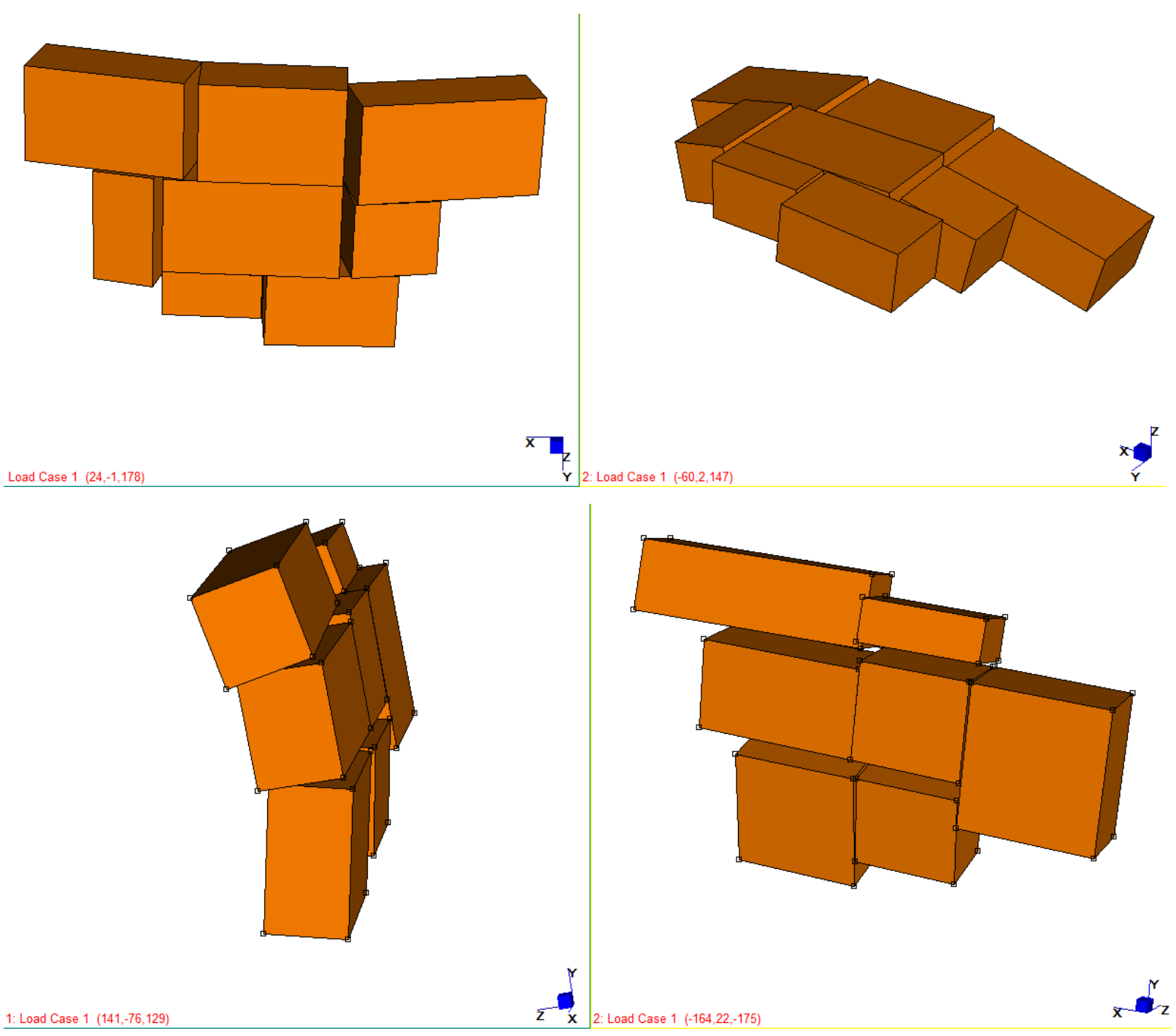

Figure 14: Case I. Some typical deformed shape at collapse obtained with the kinematic model proposed. 

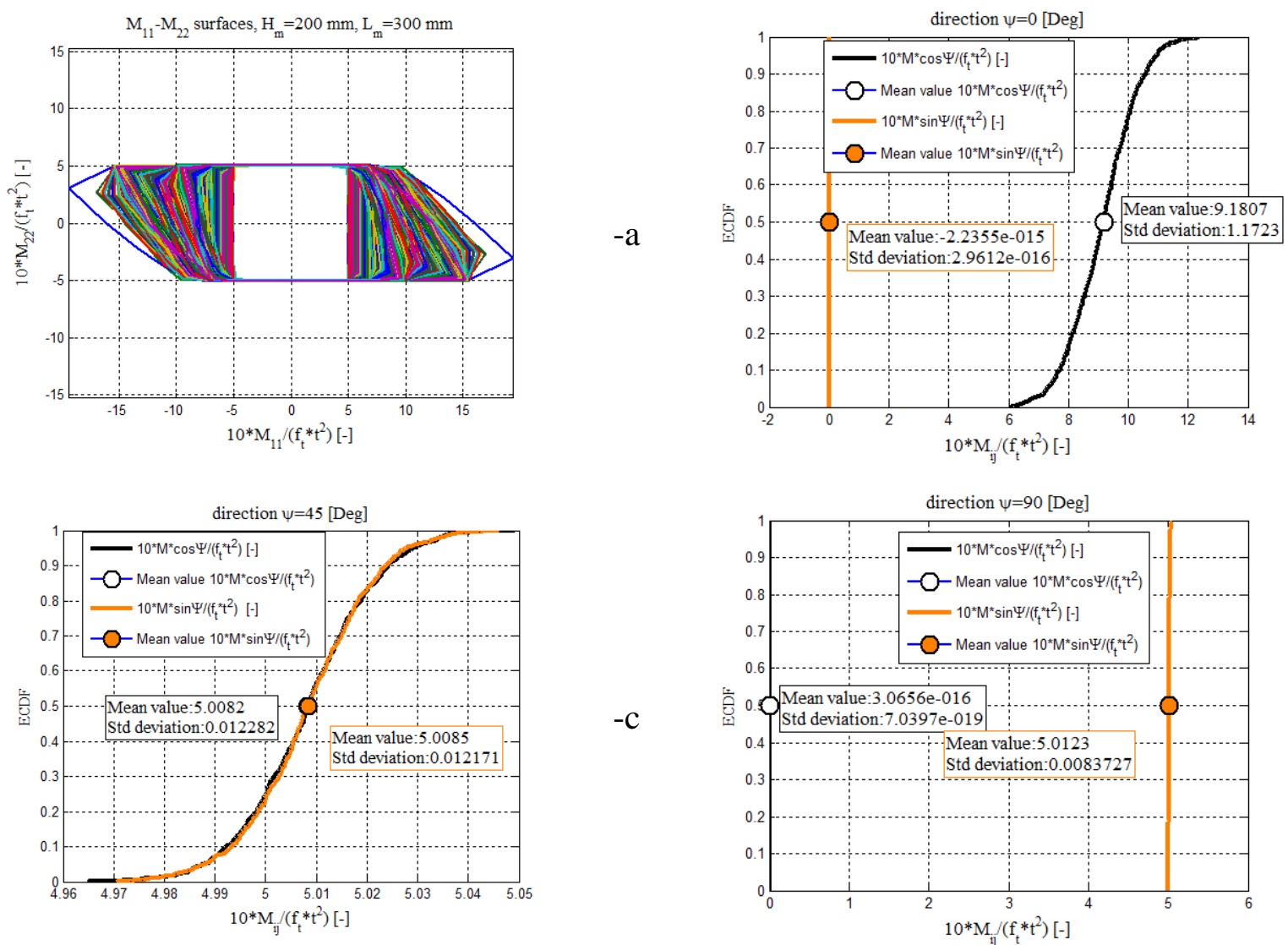

Figure 15: Case II results, $\mathrm{M}_{11}-\mathrm{M}_{22}$ sections assuming for joints a Lourenço-Rots [1] failure criterion. Monte Carlo failure surfaces and corresponding ECDF at fixed load paths given by the $\psi$ angle. 

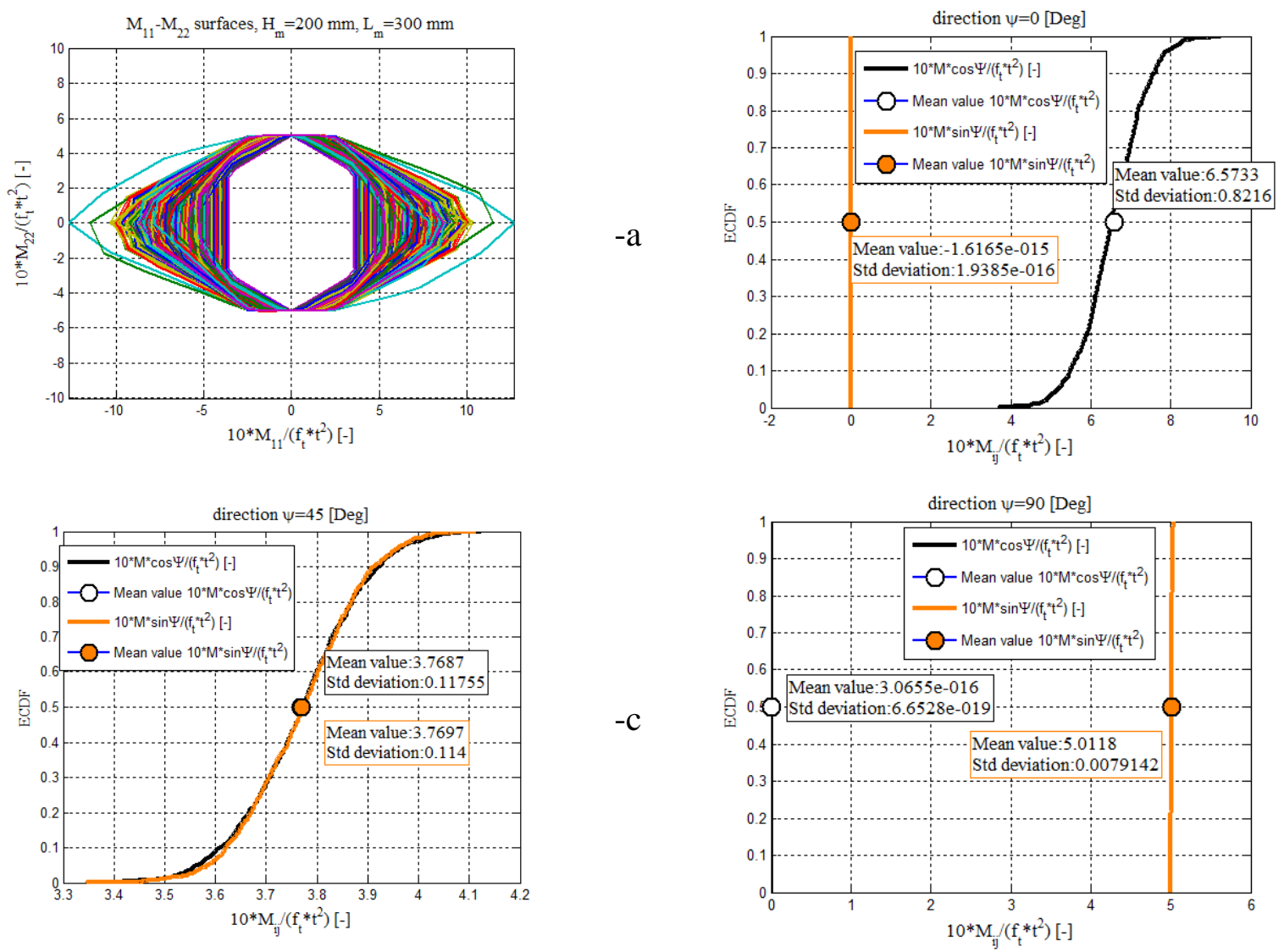

Figure 16: Case II results, $\mathrm{M}_{11}-\mathrm{M}_{12}$ sections assuming for joints a Lourenço-Rots [1] failure criterion. Monte Carlo failure surfaces and corresponding ECDF at fixed load paths given by the $\psi$ angle. 

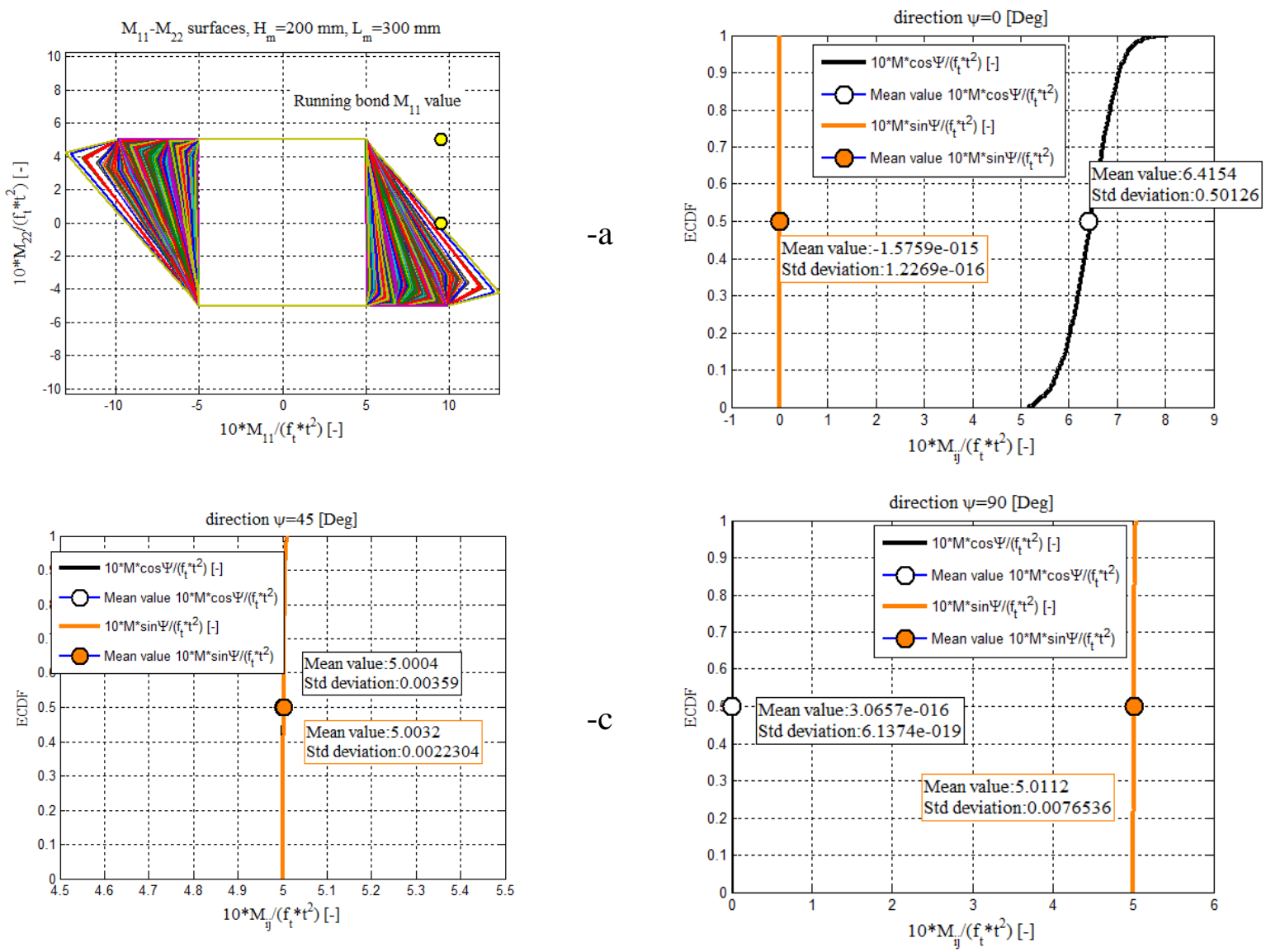

Figure 17: Case II results, $\mathrm{M}_{11}-\mathrm{M}_{22}$ sections assuming for joints a classic Mohr-Coulomb failure criterion. Monte Carlo failure surfaces and corresponding ECDF at fixed load paths given by the $\psi$ angle. 

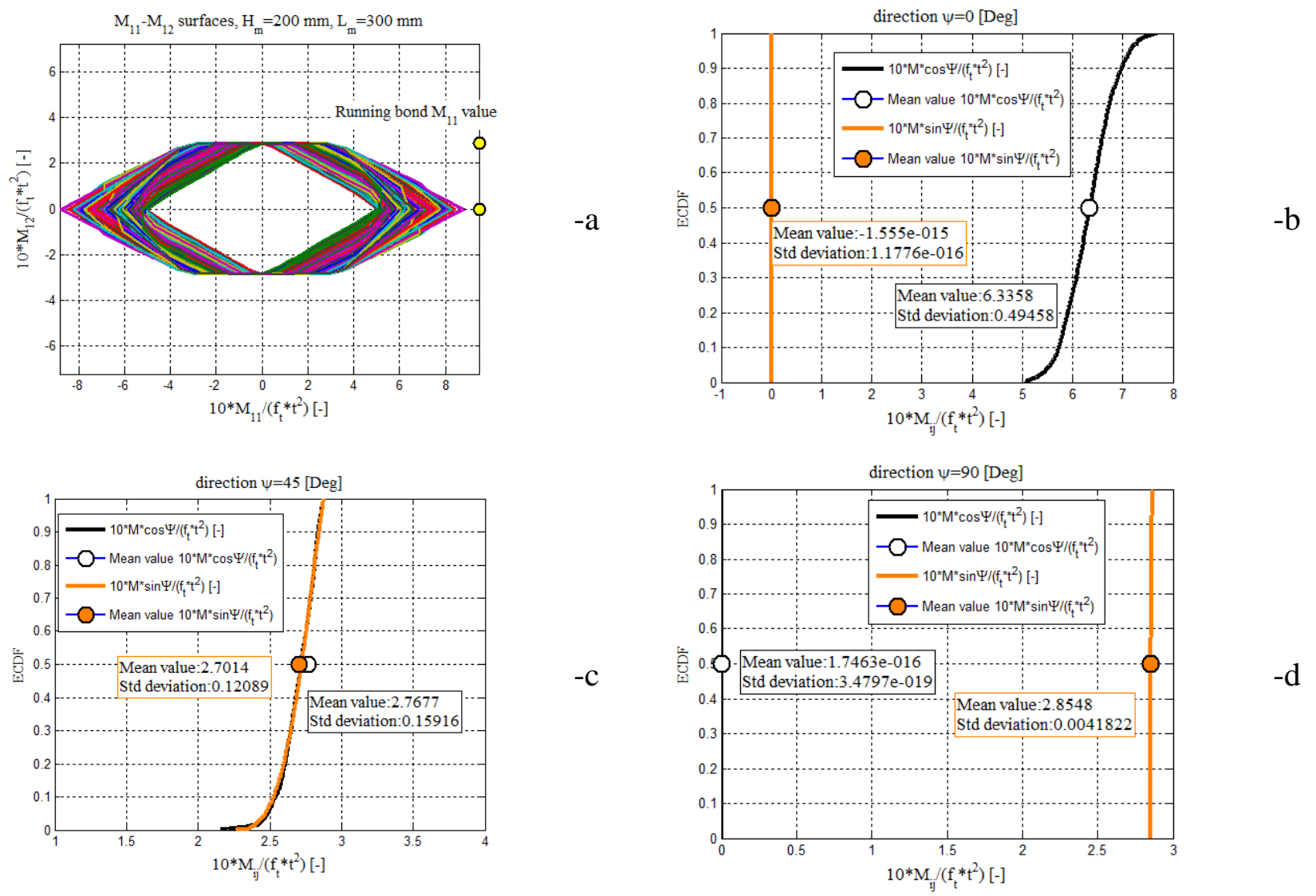

Figure 18: Case II results, $\mathrm{M}_{11}-\mathrm{M}_{12}$ sections assuming for joints a classic Mohr-Coulomb failure criterion. Monte Carlo failure surfaces and corresponding ECDF at fixed load paths given by the $\psi$ angle. 

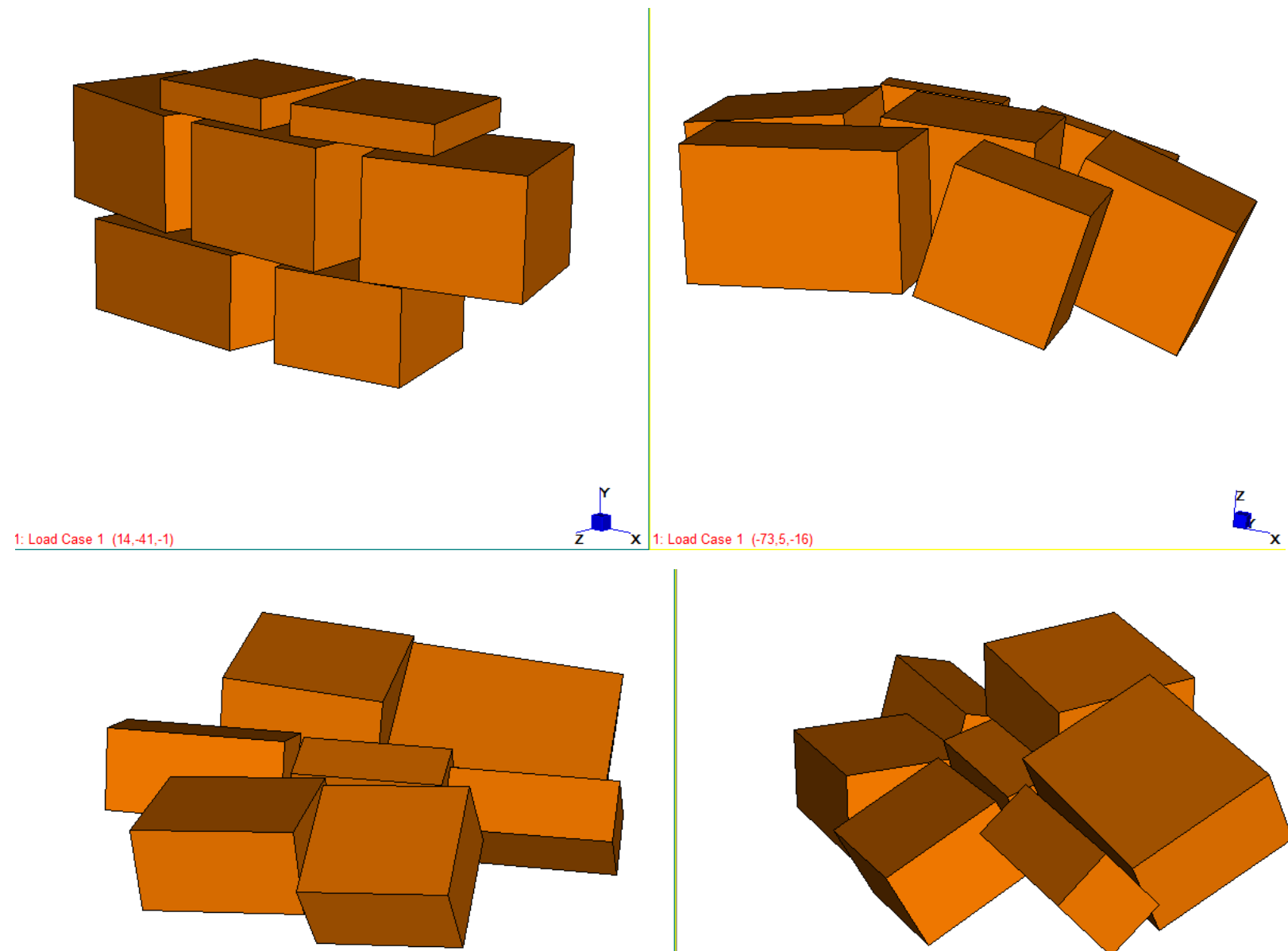

1. Load Case $1(-50,-4,168)$

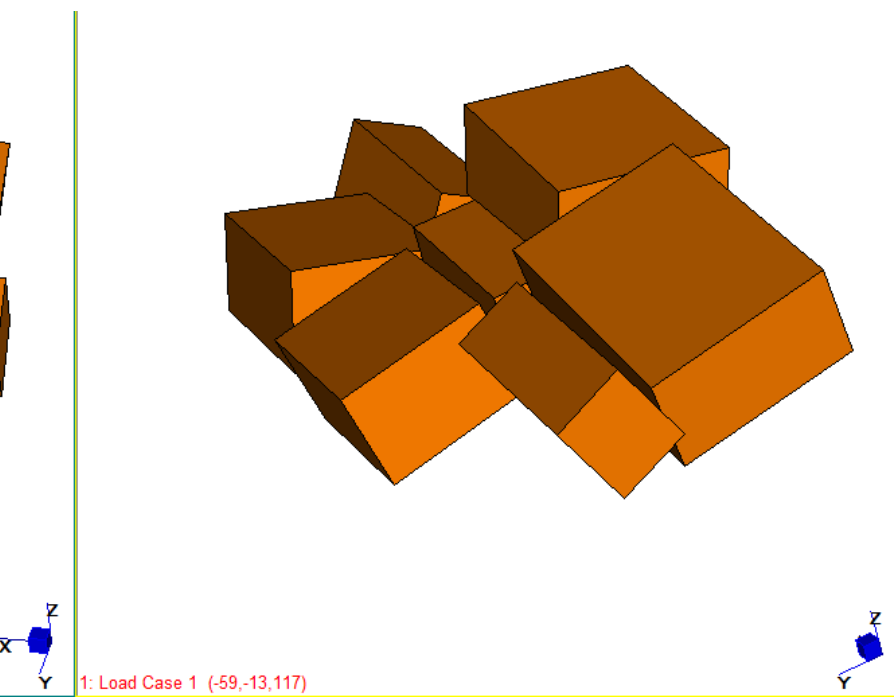

Figure 19: Case II. Some typical deformed shape at collapse obtained with the kinematic model proposed. 
Masonry panel discretized into triangular FEs

Discretization with finite thickness joints
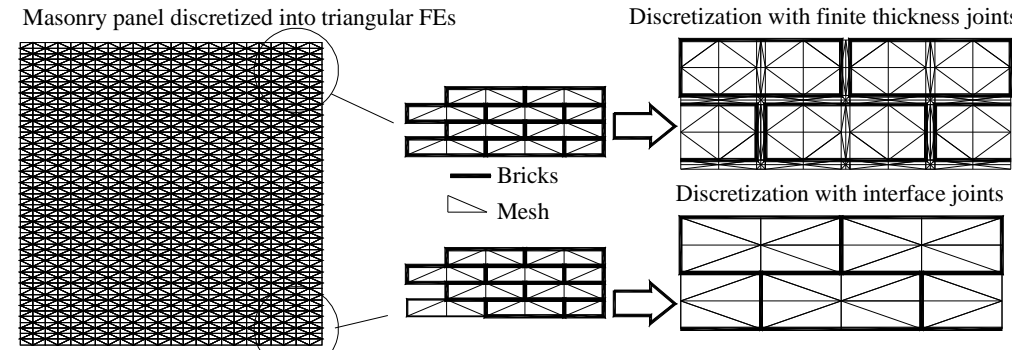

Finite thickness joints: dissipation inside joints

Interface joints: dissipation on segments (interfaces)
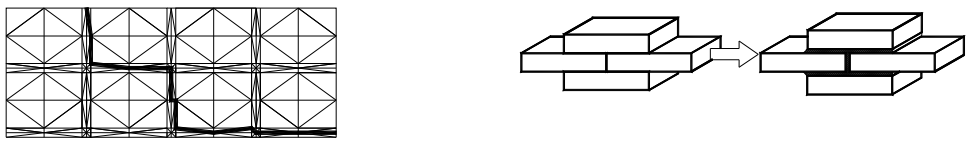

Plastic dissipation on interface $I$
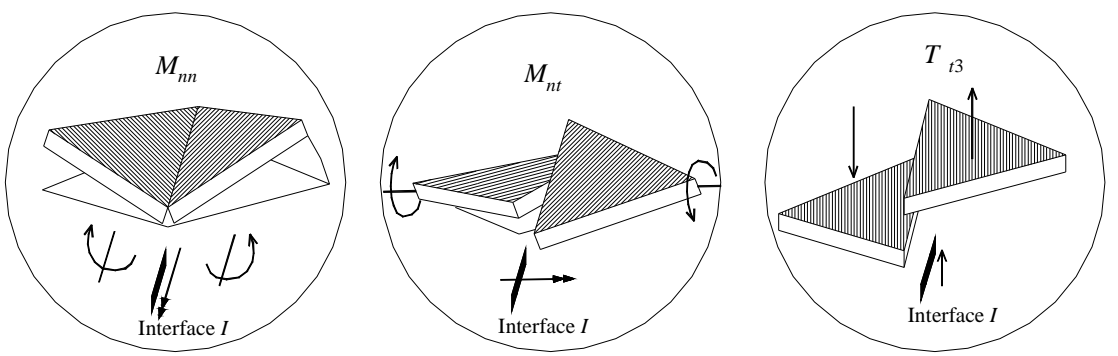

$-b$
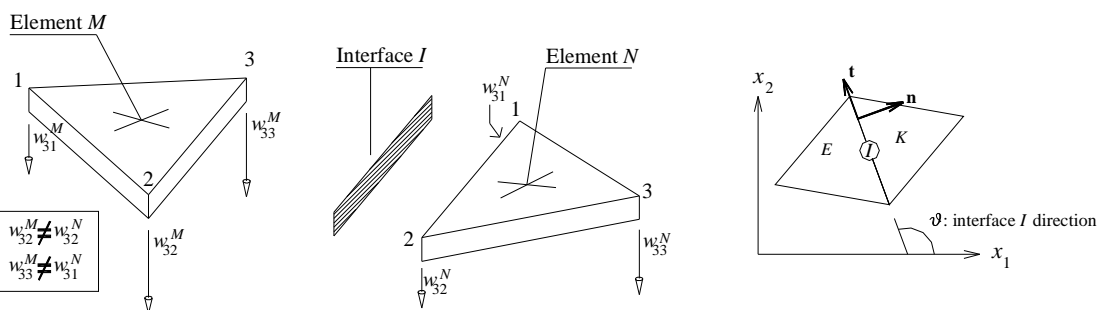

$-\mathrm{c}$

Figure 20: Triangular element utilized for a kinematic Reissner-Mindlin FE limit analysis. $-\mathrm{a}$ : possible heterogeneous discretization with finite thickness joints or joints reduced to interfaces. -b: possible plastic dissipation at the interface due to bending moment, torsion and shear. $-\mathrm{c}$ : field of velocities interpolation within each element and discontinuity at each interface between adjacent triangles. 

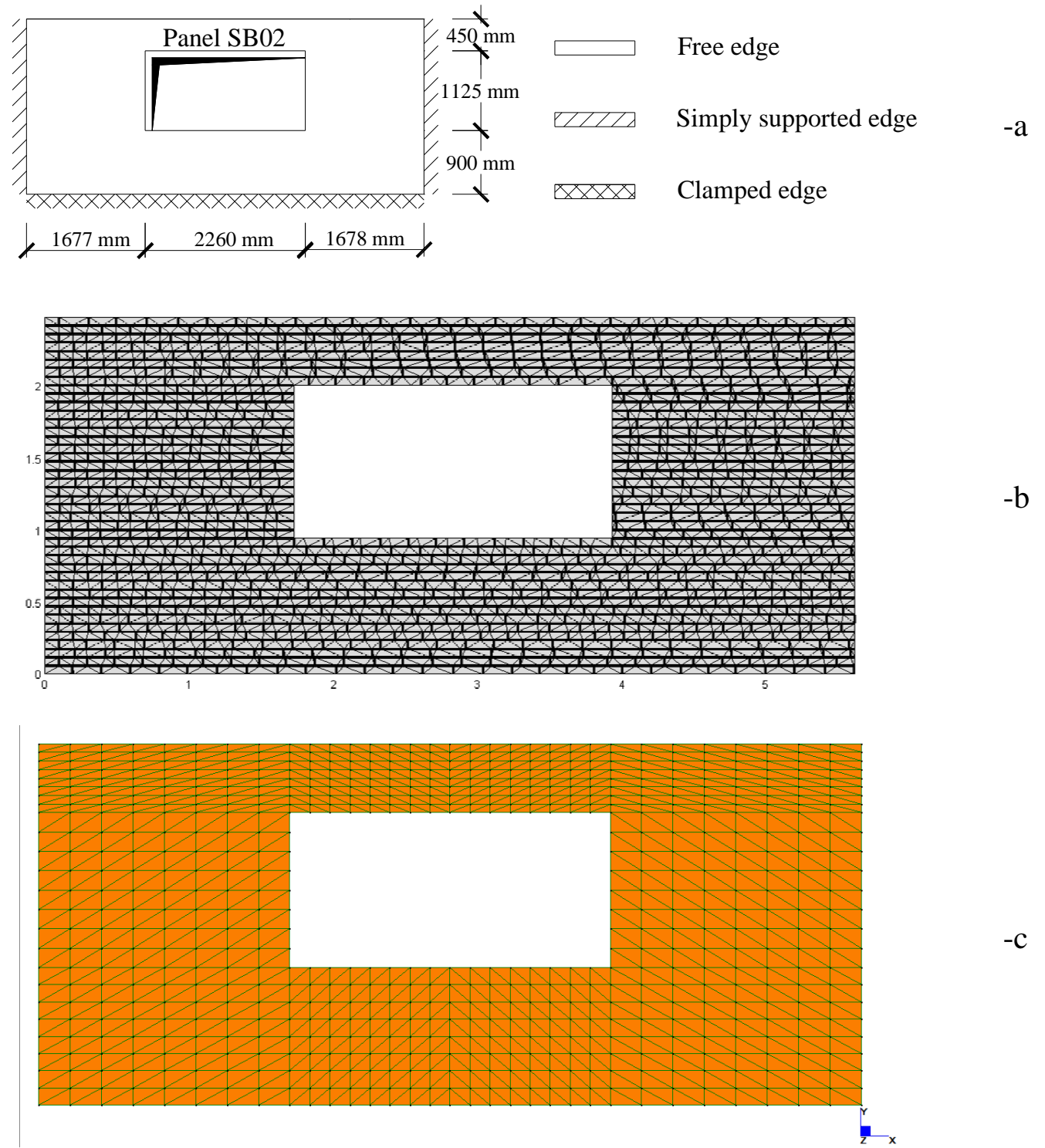

Figure 21: Windowed panel in two-way bending. -a: geometry. -b: typical heterogeneous mesh. -c: homogenized mesh. 

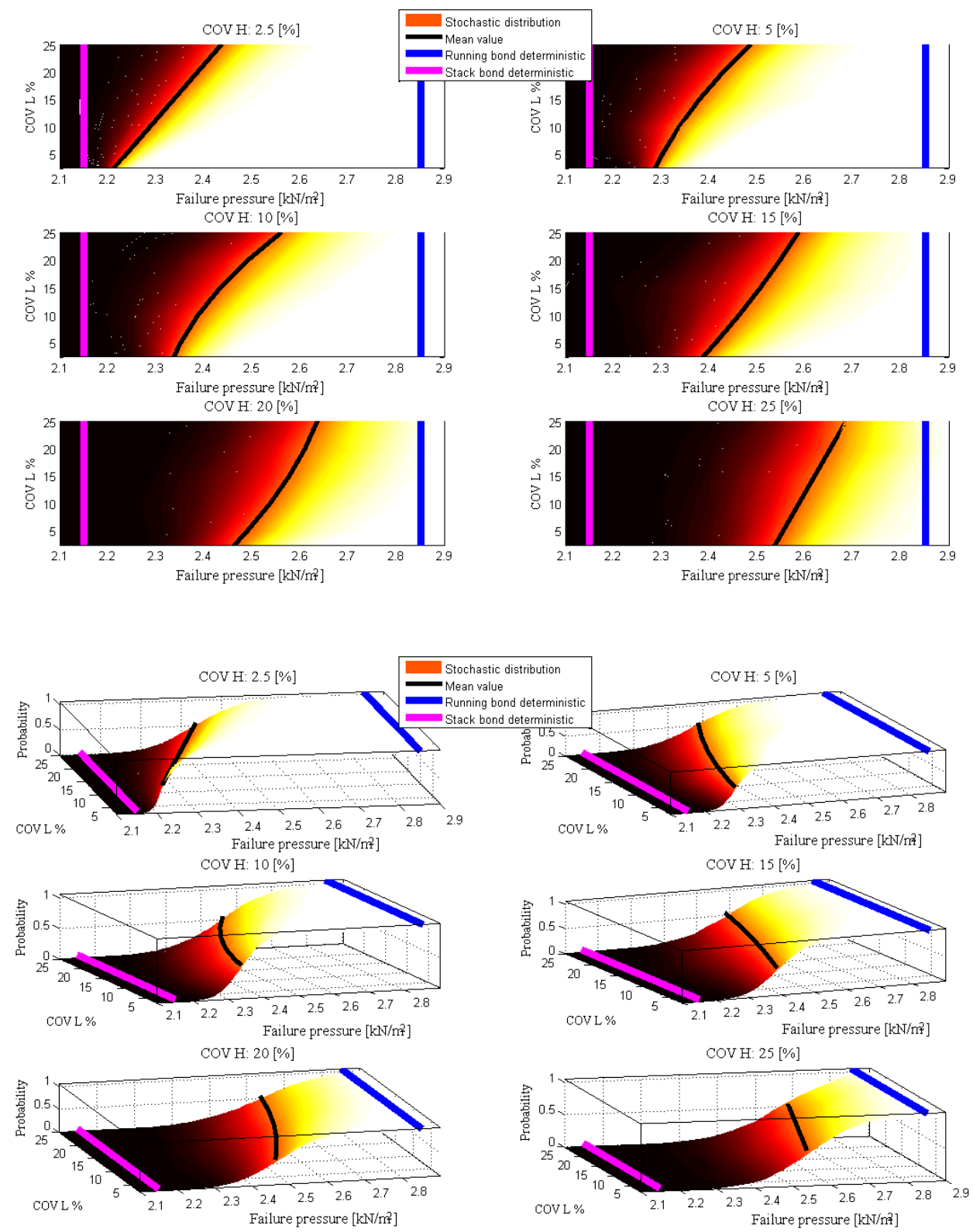

Figure 22: Windowed panel in two-way bending. Failure load distribution at different COVs of blocks length and height (Case I). 


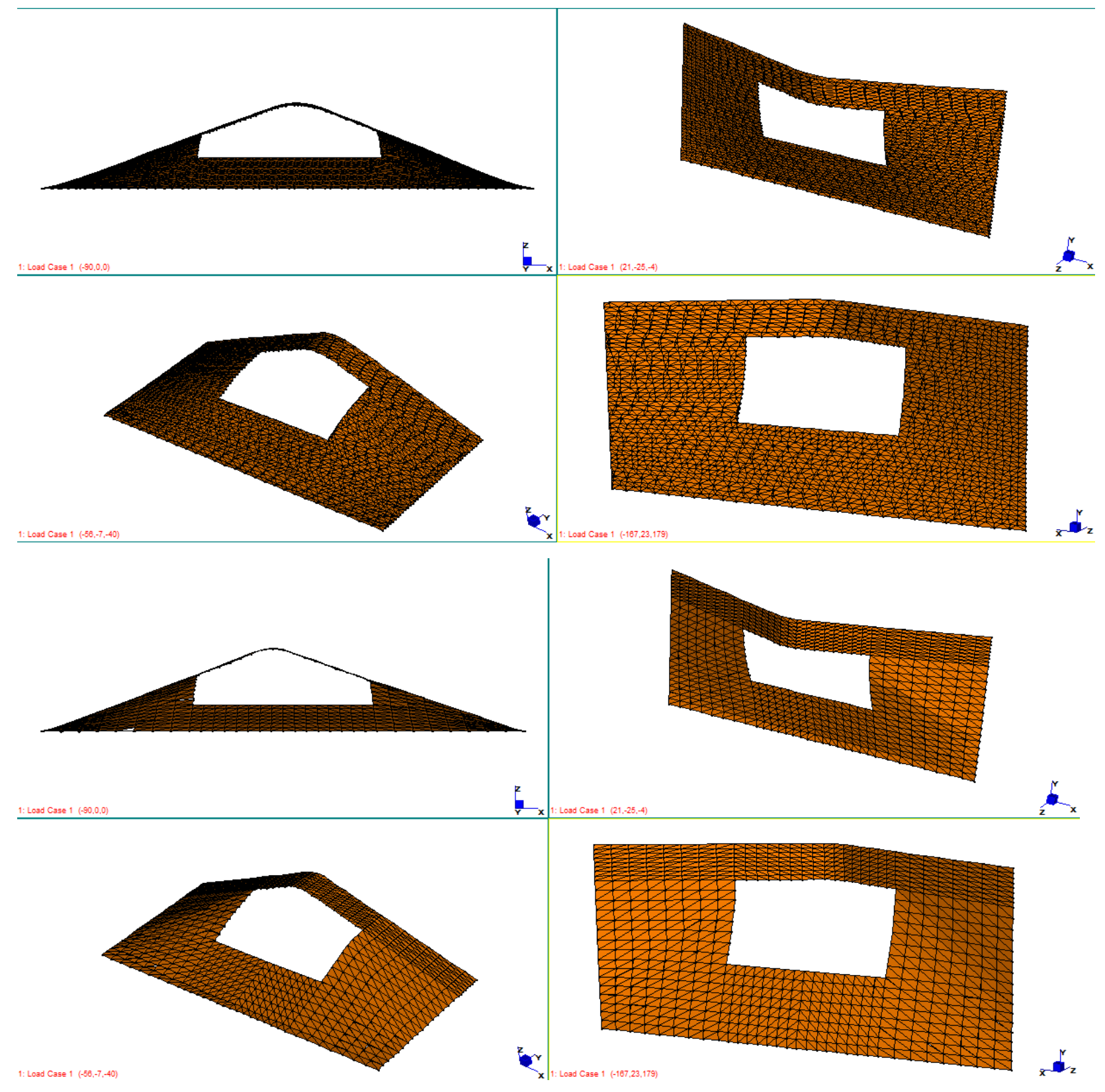

Figure 23: Windowed panel in two-way bending. Failure mechanism provided by a random heterogeneous mesh and the homogenized approach. 

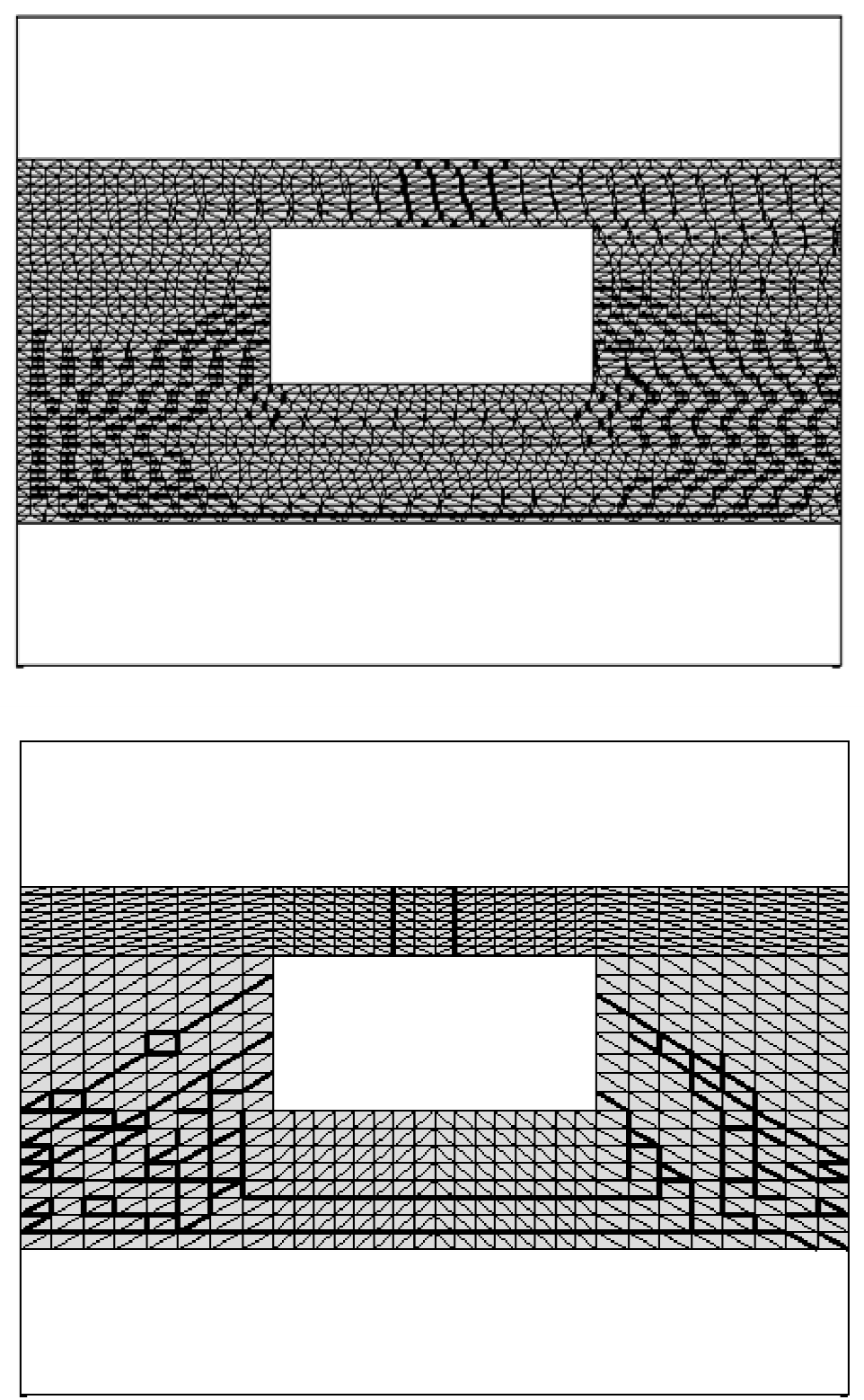

Figure 24: Windowed panel in two-way bending. Comparison between crack pattern provided by the heterogeneous (-a) and the homogenized (-b) approach in correspondence of the mean value 


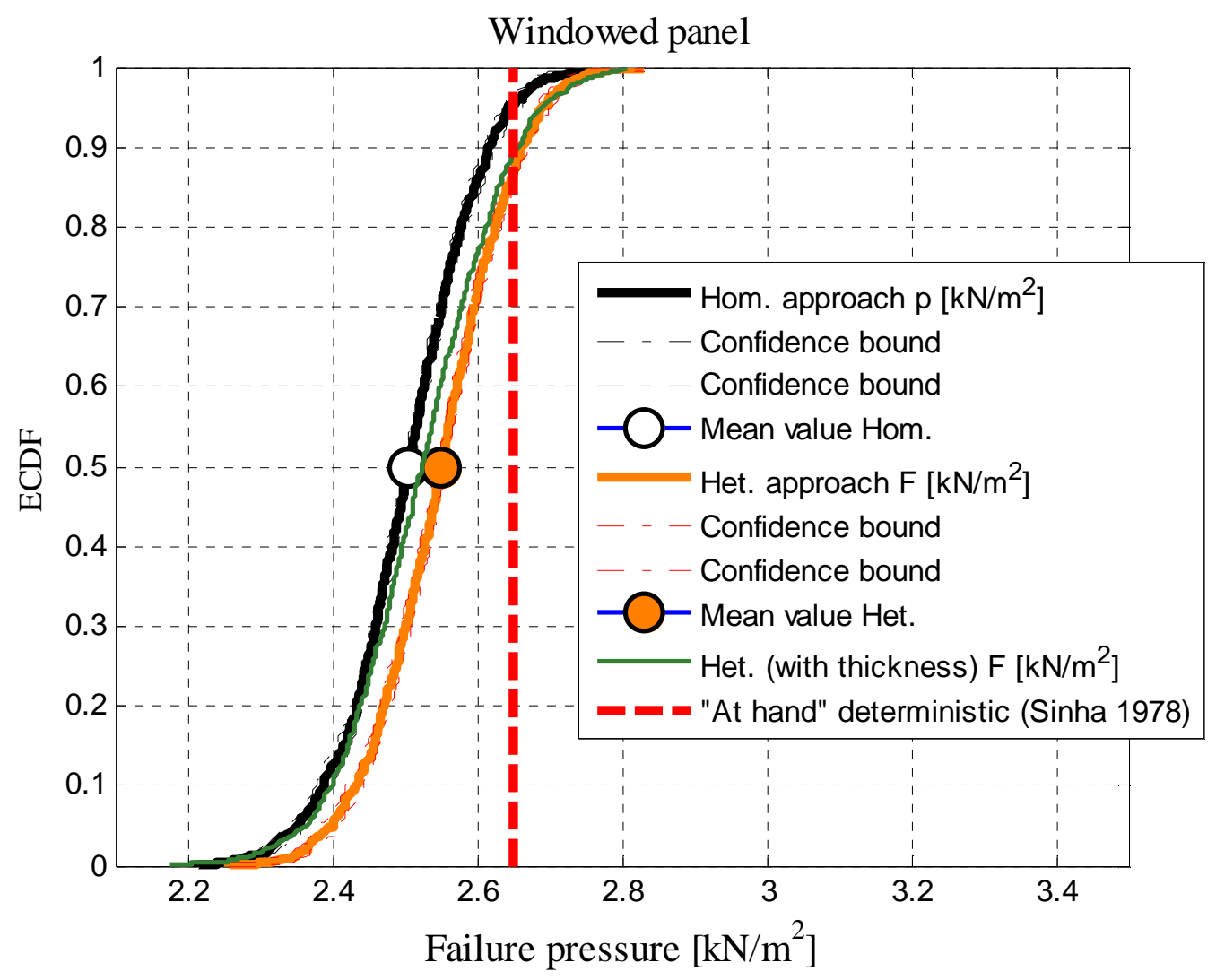

Figure 25: Windowed panel in two-way bending. ECDF of the failure load provide through a direct heterogeneous approach and homogenized limit analysis simulations. 

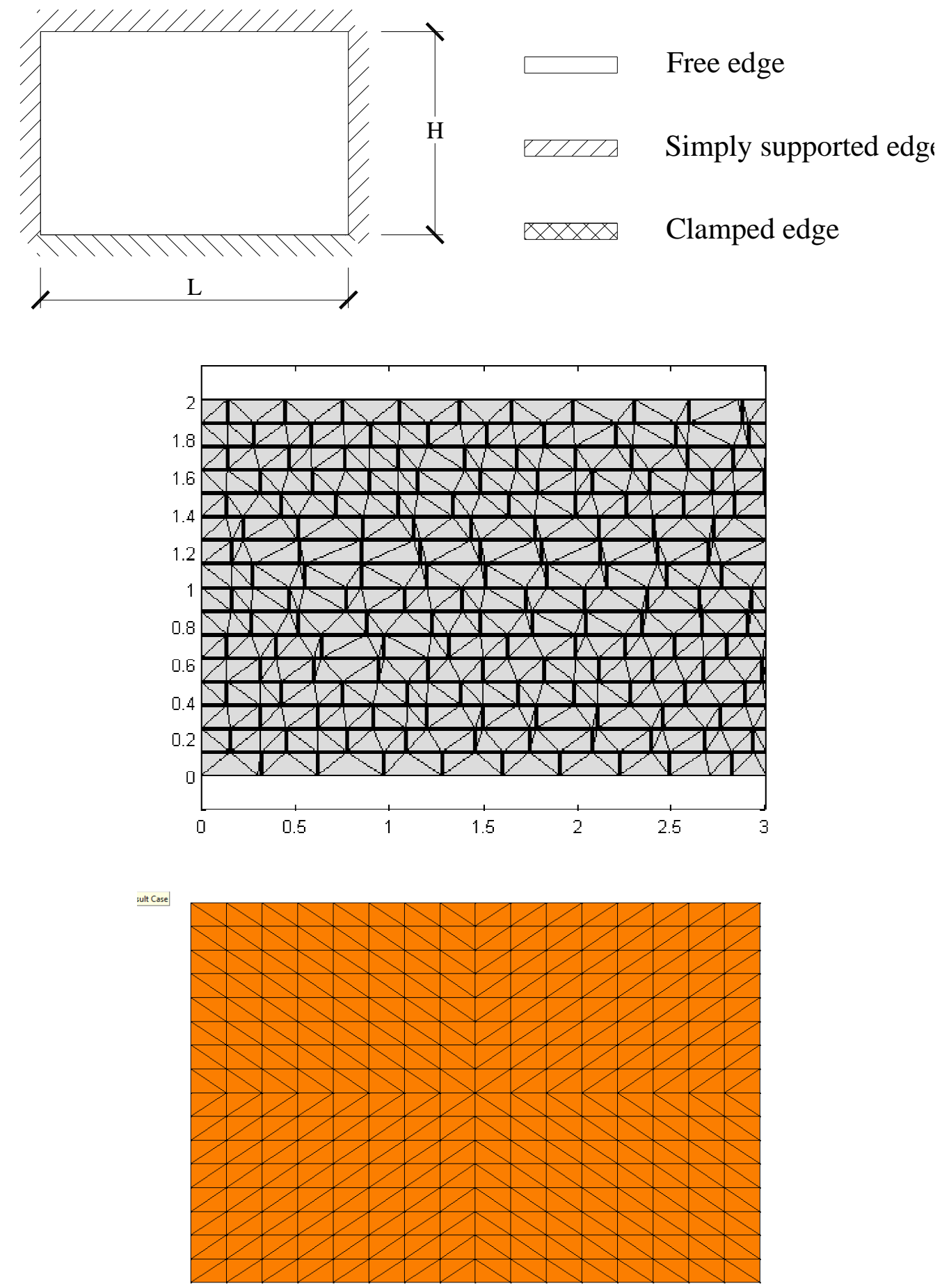

Figure 26: Out-of-plane loaded rectangular panel. Geometry, loading and constraints condition, typical heterogeneous and homogenized mesh used. 

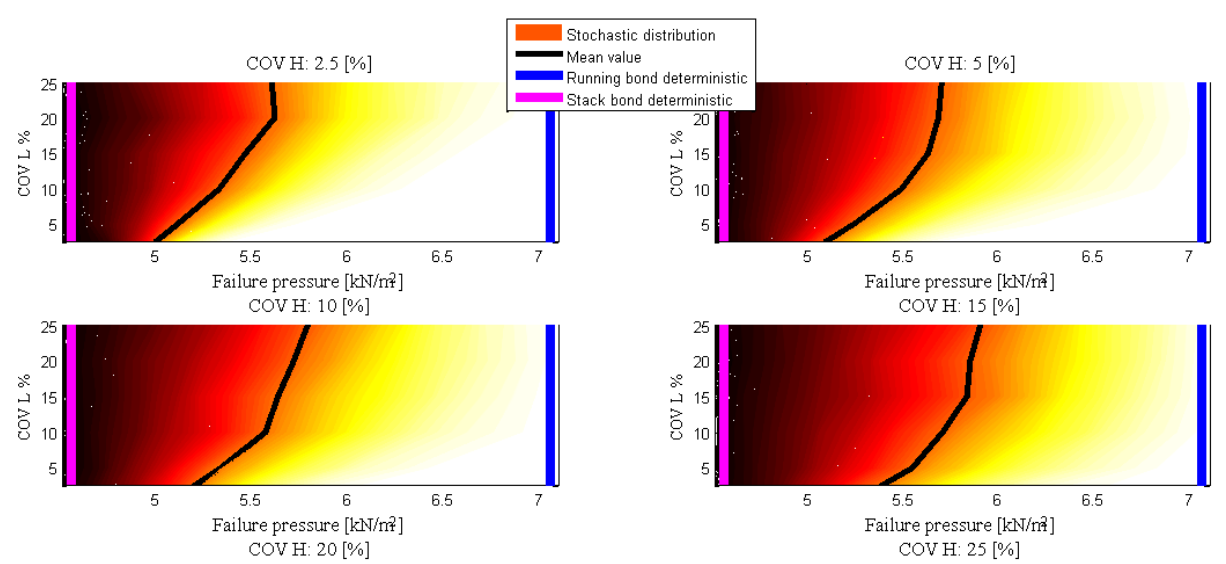

$-\mathrm{a}$
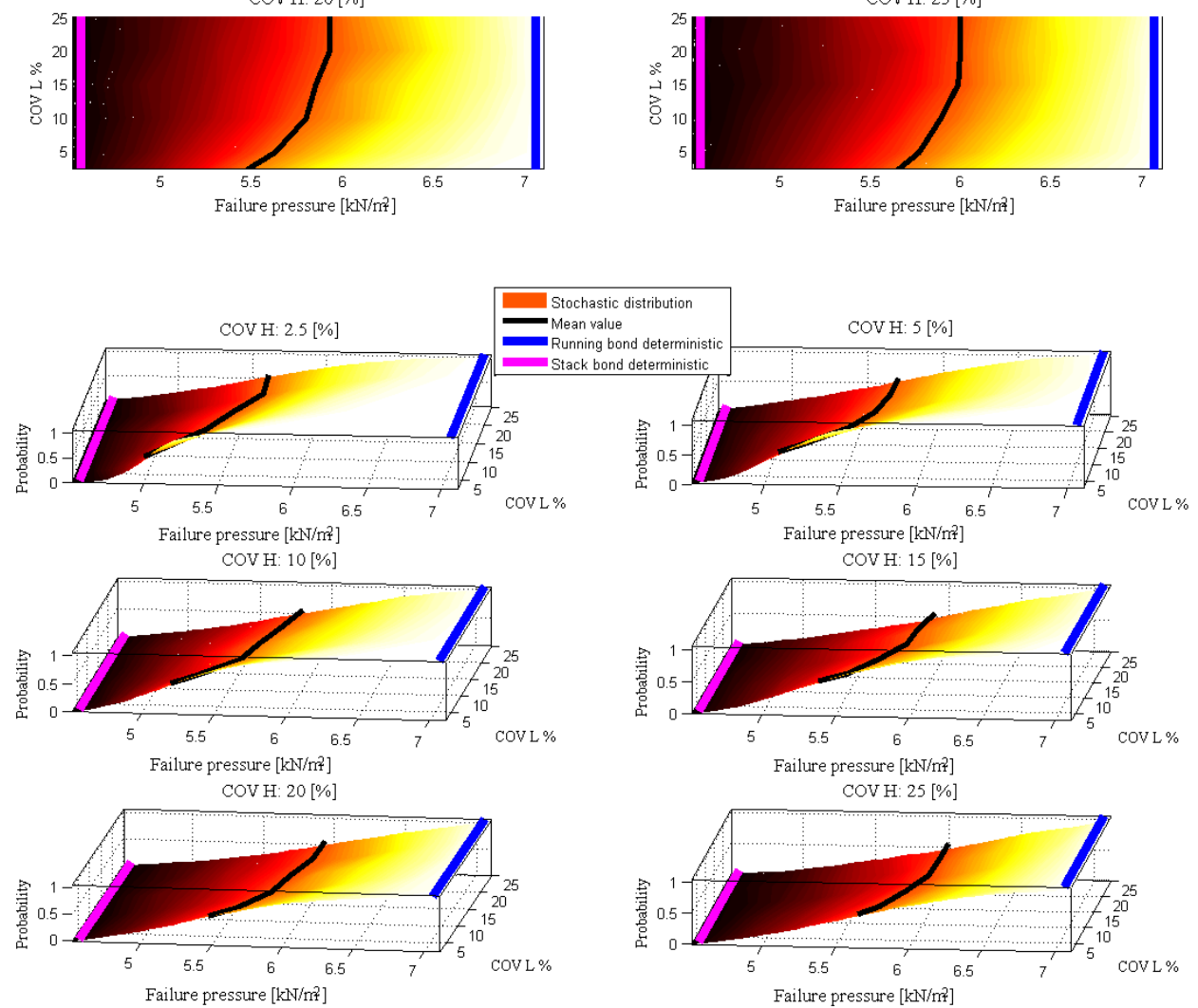

$-b$

Figure 27: Out-of-plane loaded rectangular panel. Failure load distribution at different COVs of blocks length and height (Case I). 


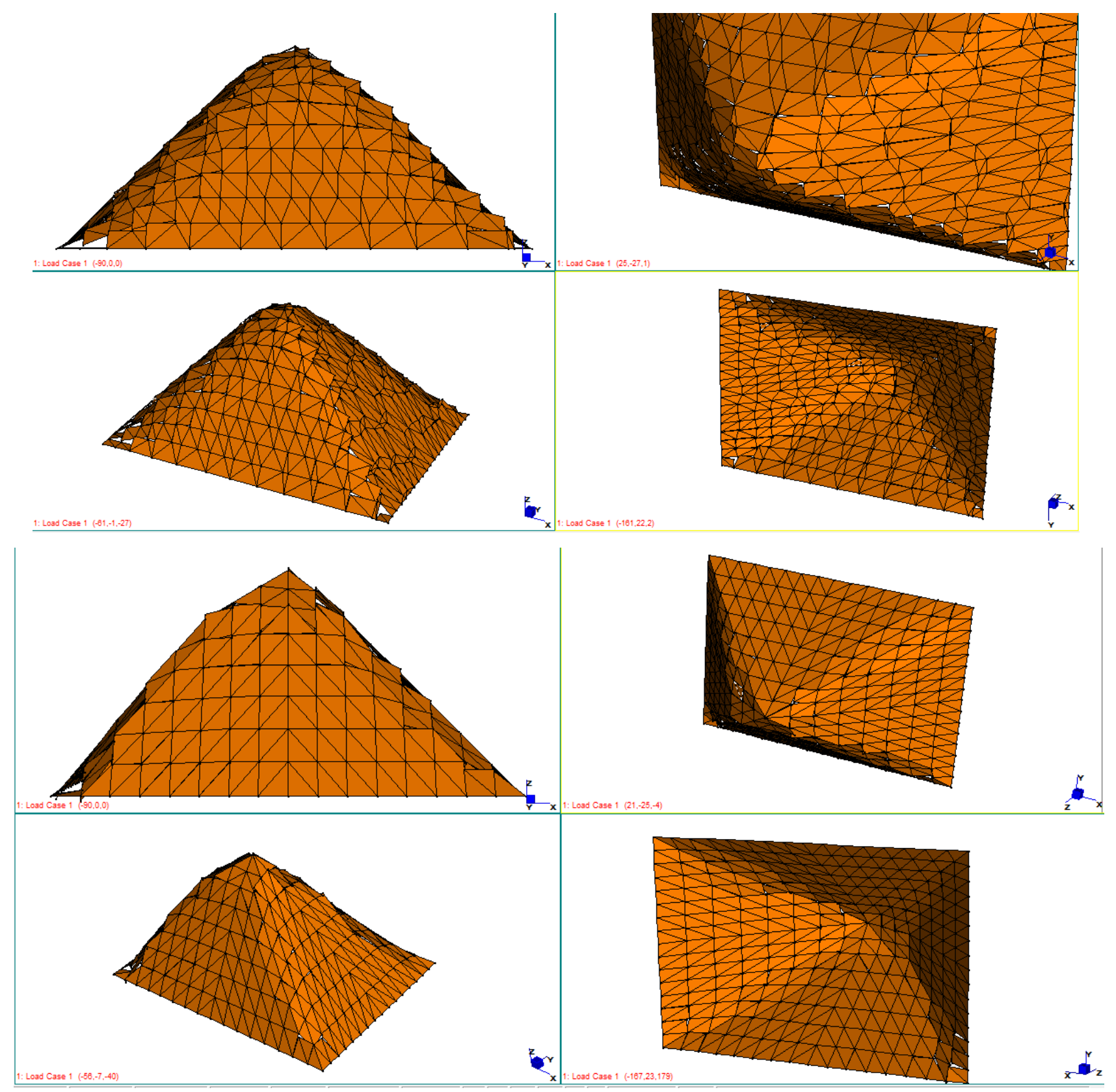

Figure 28: Out-of-plane loaded rectangular panel. Failure mechanism provided by a random heterogeneous mesh and the homogenized approach. 

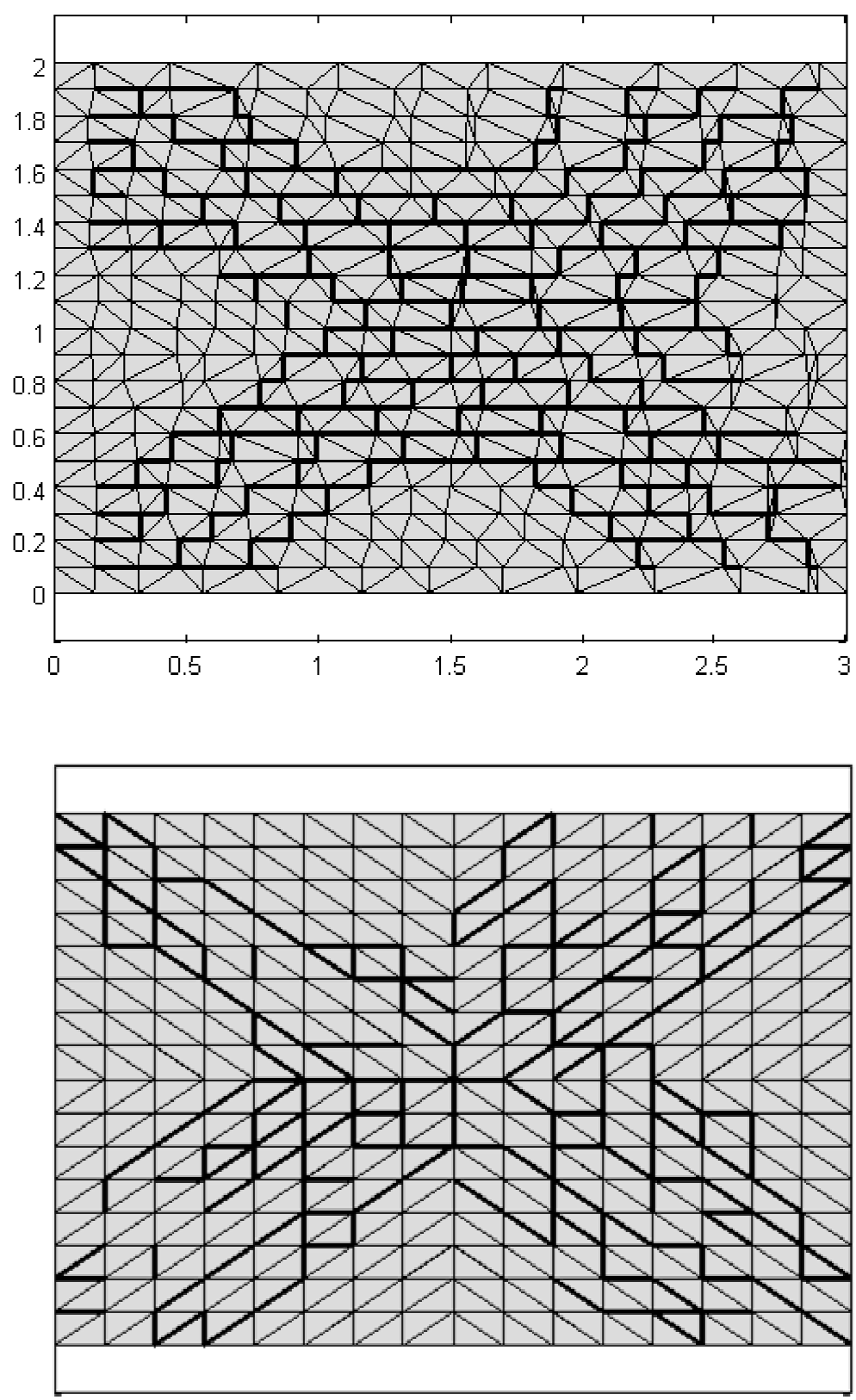

Figure 29: Out-of-plane loaded rectangular panel. Comparison between crack pattern provided by the heterogeneous (-a) and the homogenized (-b) approach in correspondence of the mean value 


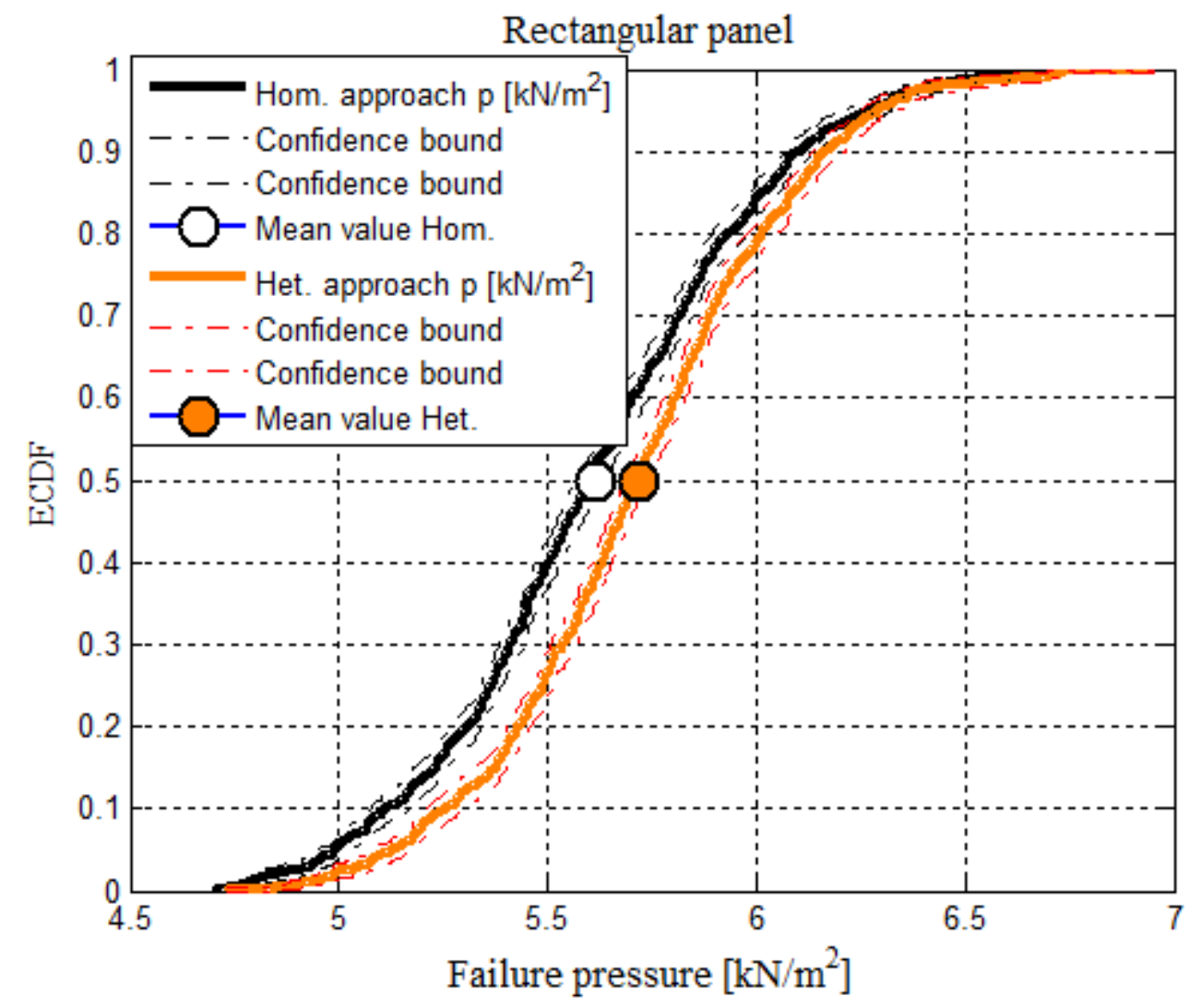

Figure 30: Out-of-plane loaded square panel. ECDF of the failure load provide through a direct heterogeneous approach a homogenized limit analysis simulations.

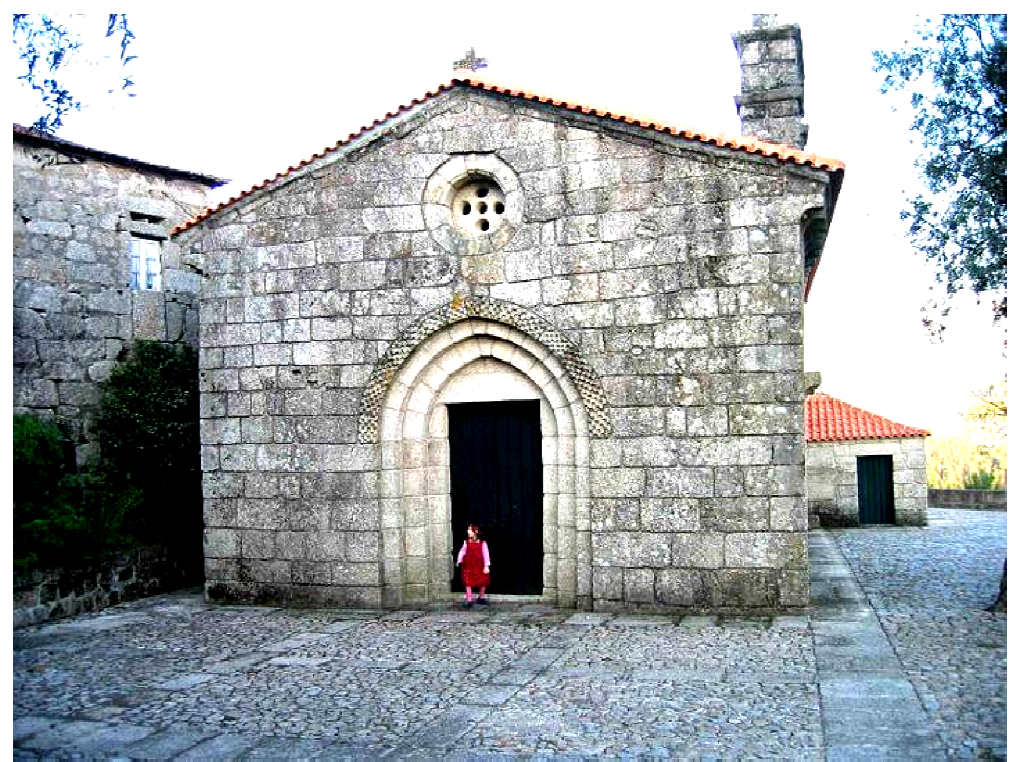

Figure 31: Façade of the Church of Gondar (Portugal). 


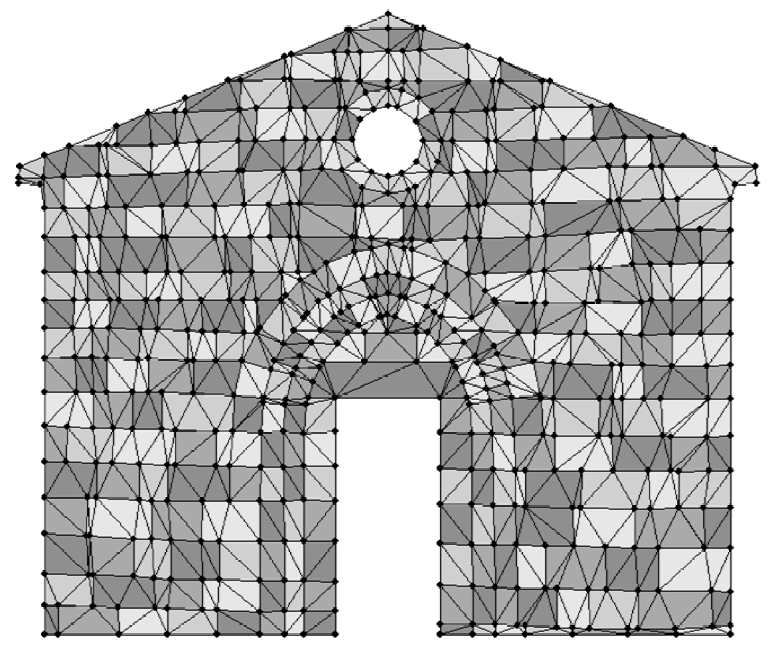

$-a$

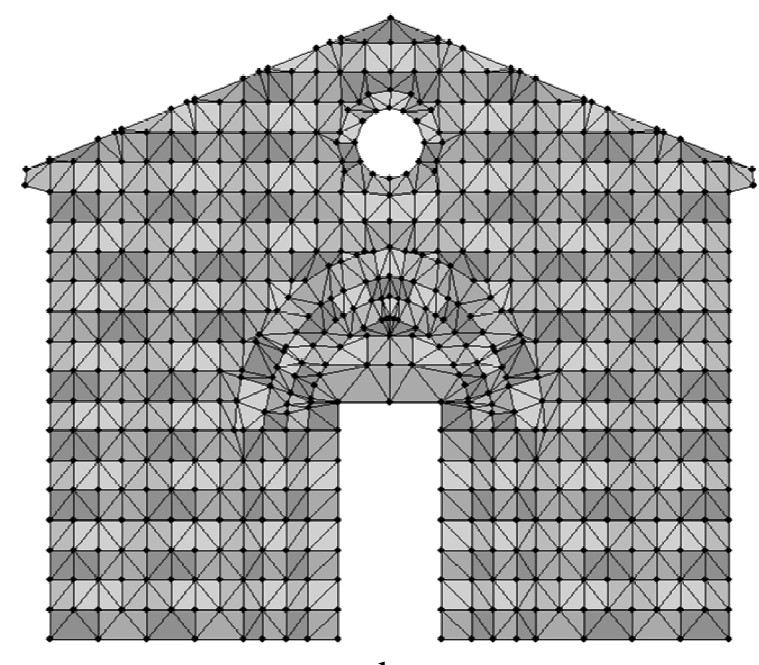

$-b$

Figure 32: Church of Gondar, FE discretization adopted. -a: heterogeneous random mesh. -b: running bond regular heterogeneous mesh and homogenized random mesh.

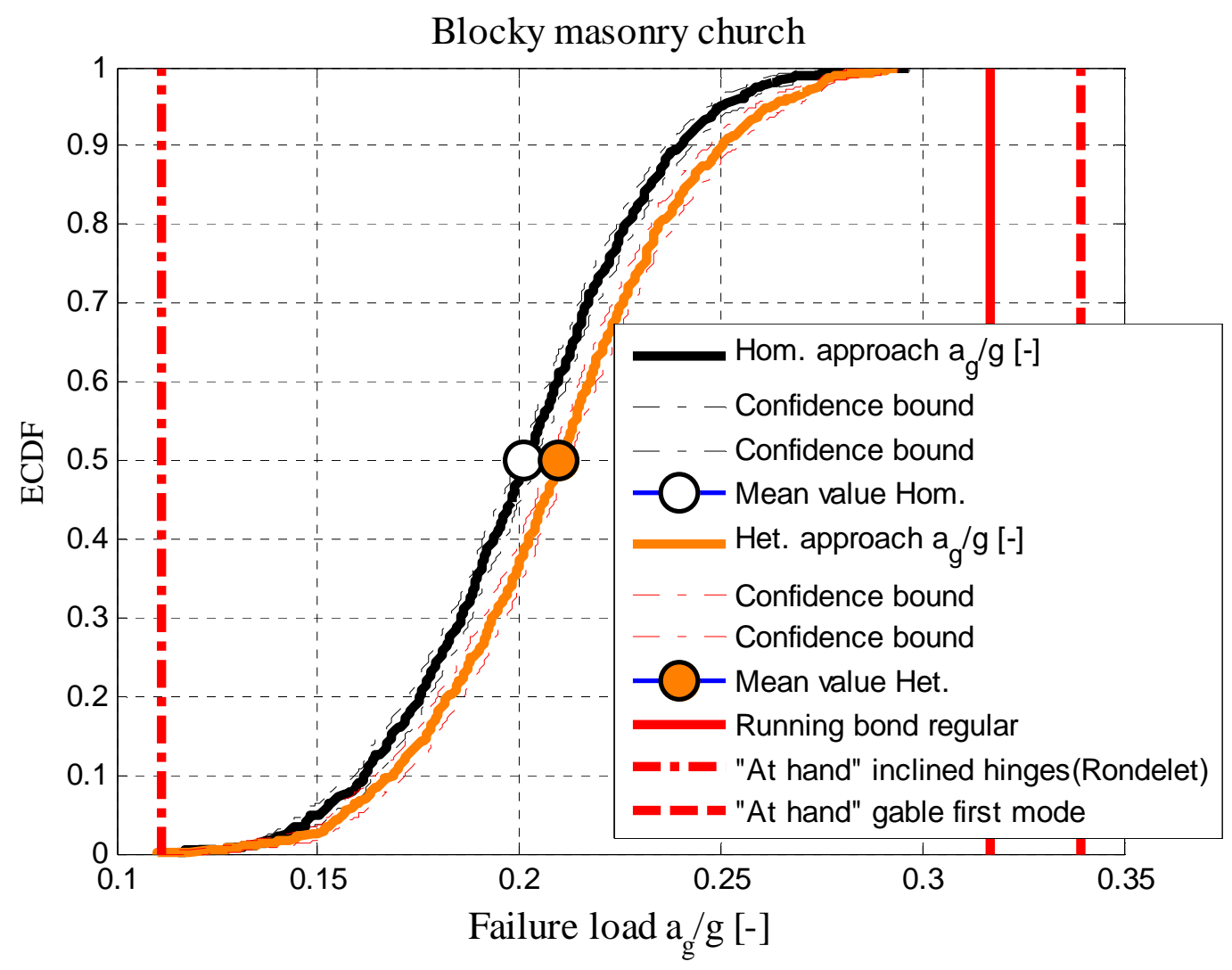

Figure 33: Church of Gondar, ECDF of the failure load provide through a direct heterogeneous approach and homogenized limit analysis simulations. Deterministic values found using hand failure mechanisms and assuming a running bond disposition are also reported. 

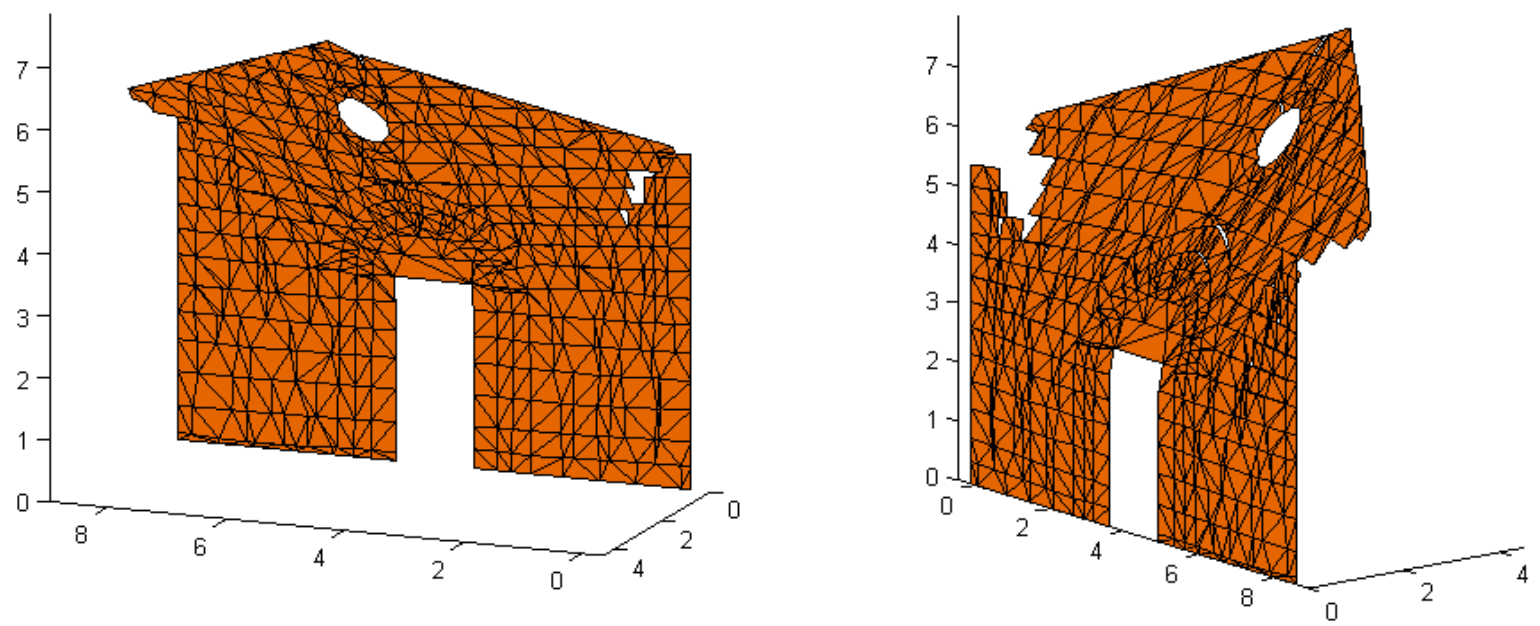

-a random pattern ag/g=0.209
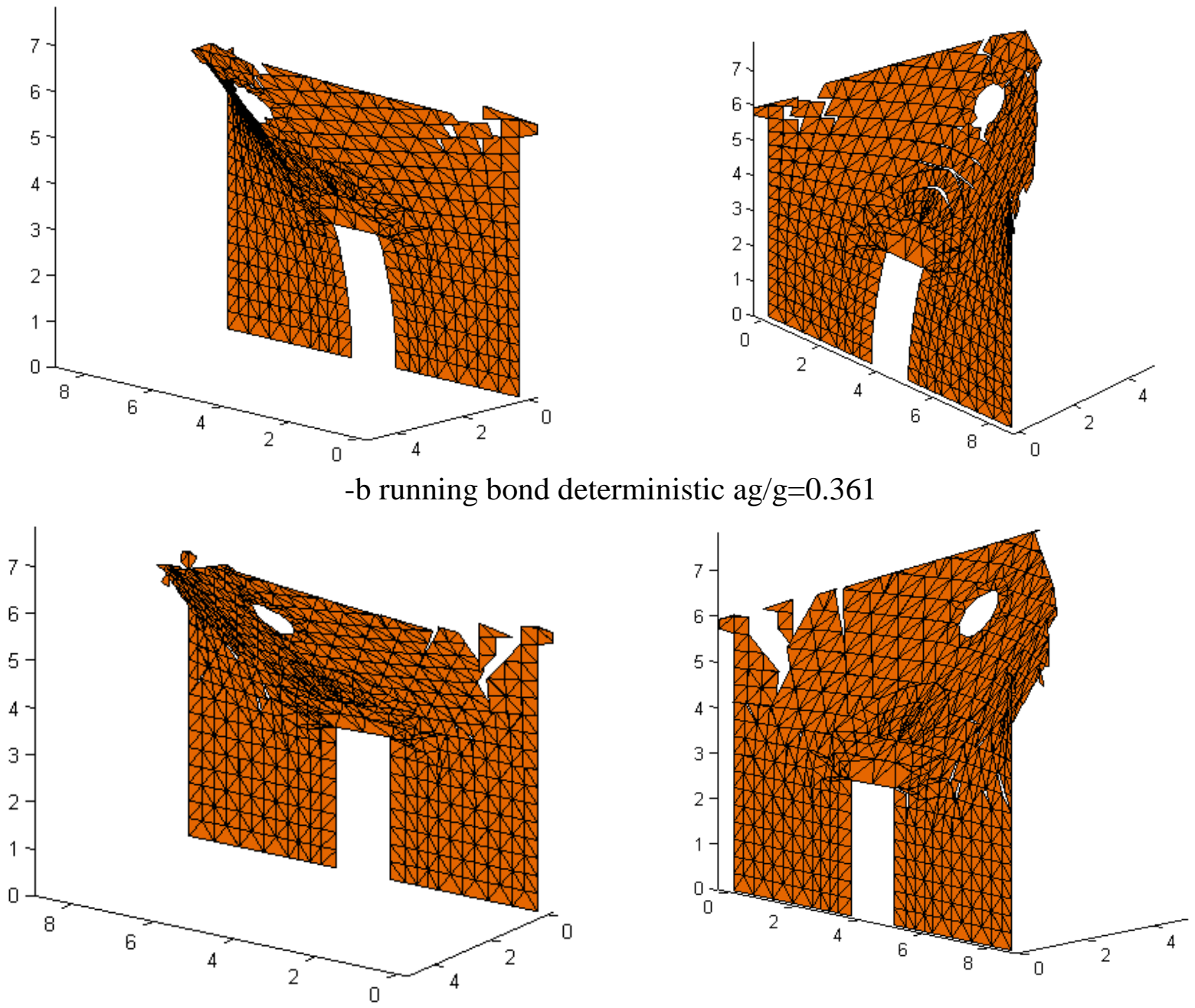

-c: homogenized random ag/g=0.201

Figure 34: Church of Gondar, failure mechanisms and collapse loads provided by a random heterogeneous mesh (-a), a deterministic running bond pattern $(-b)$ and the homogenized approach (c). 


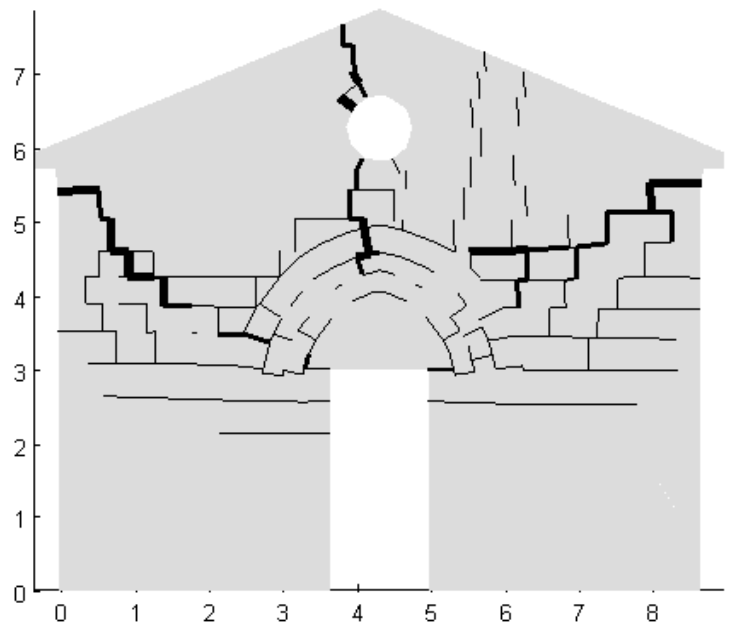

$-\mathrm{a}$

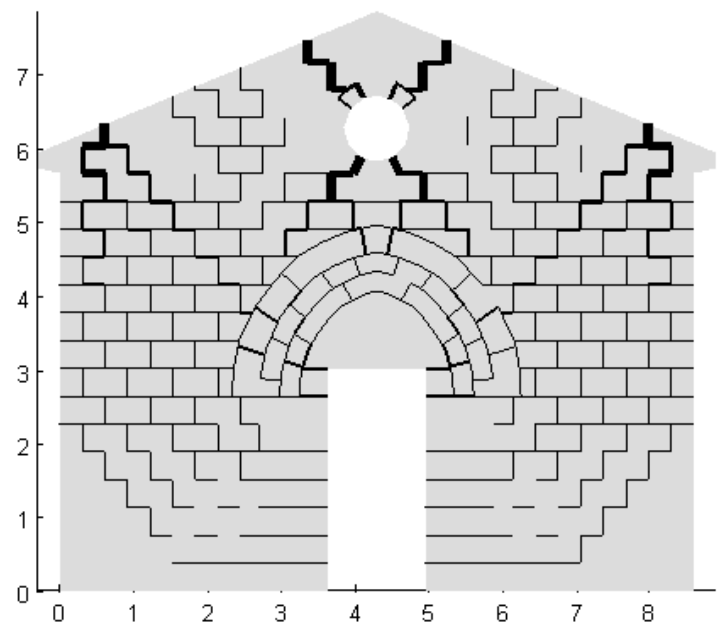

$-b$

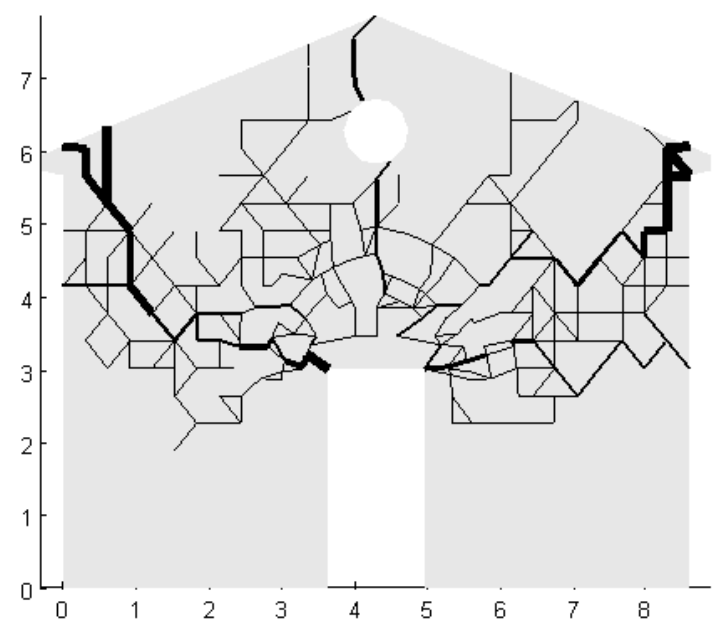

$-\mathrm{c}$

Figure 35: Church of Gondar, plastic dissipation on interfaces provided by a random heterogeneous mesh (-a), a deterministic running bond pattern (b) and the homogenized approach (-c). 


\section{Tables}

Table I: Representative element of volume level. Mechanical characteristics assumed for mortar joints reduced to interfaces $\left(f_{t}\right.$ : tension cut-off, $c$ : cohesion, $\Phi$ : friction angle, $f_{c}:$ compressive strength, $\Phi_{2}$ : shape of the linearized compressive cap).

\begin{tabular}{|c|c|c|c|c|}
\hline$f_{t} \mathrm{MPa}$ & $c$ & $\Phi$ & $f_{c} \mathrm{MPa}$ & $\Phi_{2}$ \\
\hline 0.05 & $1.4 f_{t}$ & $37^{\circ}$ & 1.5 & $60^{\circ}$ \\
\hline
\end{tabular}

Table II: Structural level, windowed panel. Mechanical characteristics assumed for mortar joints reduced to interfaces $\left(f_{t}\right.$ : tension cut-off, $c$ : cohesion, $\Phi$ : friction angle, $f_{c}$ : compressive strength, $\Phi_{2}$ : shape of the linearized compressive cap).

\begin{tabular}{|c|c|c|c|c|}
\hline$f_{t} \mathrm{MPa}$ & $c$ & $\Phi$ & $f_{c} \mathrm{MPa}$ & $\Phi_{2}$ \\
\hline 0.30 & $1.0 f_{t}$ & $36^{\circ}$ & 5 & $60^{\circ}$ \\
\hline
\end{tabular}

Table III: Structural level, rectangular panel out-of-plane loaded. Mechanical characteristics assumed for mortar joints reduced to interfaces $\left(f_{t}\right.$ : tension cut-off, $c$ : cohesion, $\Phi$ : friction angle, $f_{c}$ : compressive strength, $\Phi_{2}$ : shape of the linearized compressive cap).

\begin{tabular}{|c|c|c|c|c|}
\hline$f_{t} \mathrm{MPa}$ & $c$ & $\Phi$ & $f_{c} \mathrm{MPa}$ & $\Phi_{2}$ \\
\hline 0.07 & $1.4 f_{t}$ & $37^{\circ}$ & 3 & $60^{\circ}$ \\
\hline
\end{tabular}

